
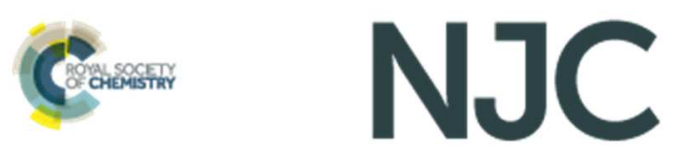

\title{
Sensing of Diclofenac by a Porphyrin-based Artificial Receptor
}

\begin{tabular}{|r|l|}
\hline Journal: & New Journal of Chemistry \\
\hline Manuscript ID & NJ-ART-06-2018-002737.R2 \\
\hline Article Type: & Paper \\
\hline Date Submitted by the Author: & O7-Aug-2018 \\
\hline Complete List of Authors: & $\begin{array}{l}\text { Gallo, Emma; University of Milan, of Chemistry } \\
\text { Intrieri, Daniela; università, } \\
\text { Damiano, Caterina; università, Department of Chemistry } \\
\text { Rizzato, Silvia; Università di Milano, Deparment of Chemistry } \\
\text { Paolesse, Roberto; Universita degli Studi di Roma Tor Vergata, Chemical } \\
\text { Science and Technologies } \\
\text { Venanzi, M.; Universita degli Studi di Roma La Sapienza Facolta di Scienze } \\
\text { Matematiche Fisiche e Naturali, } \\
\text { Monti, Donato; Universita degli Studi di Roma Tor Vergata, Department of } \\
\text { Chemical Science and Technologies } \\
\text { Savioli, Marco; Universita degli Studi di Roma Tor Vergata, Science and } \\
\text { Chemical Technologies } \\
\text { Stefanelli, Manuela; University of Rome , Chemical Science and Technology }\end{array}$ \\
\hline
\end{tabular}




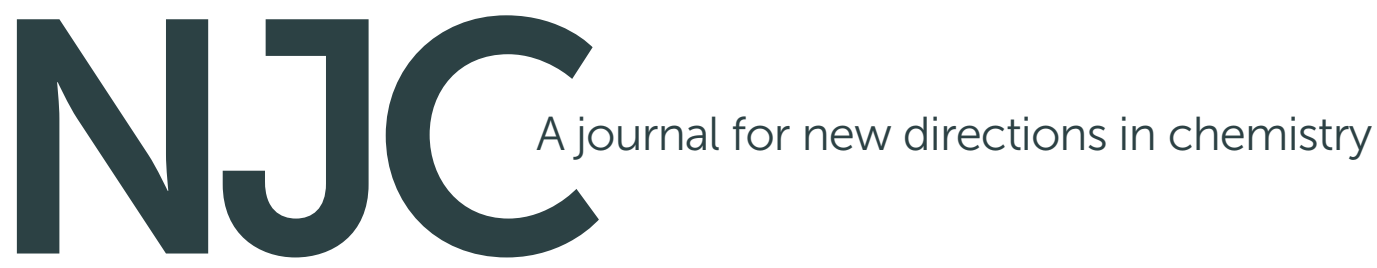

New Journal of Chemistry

ISI impact Factor (published in 2016): 3.277

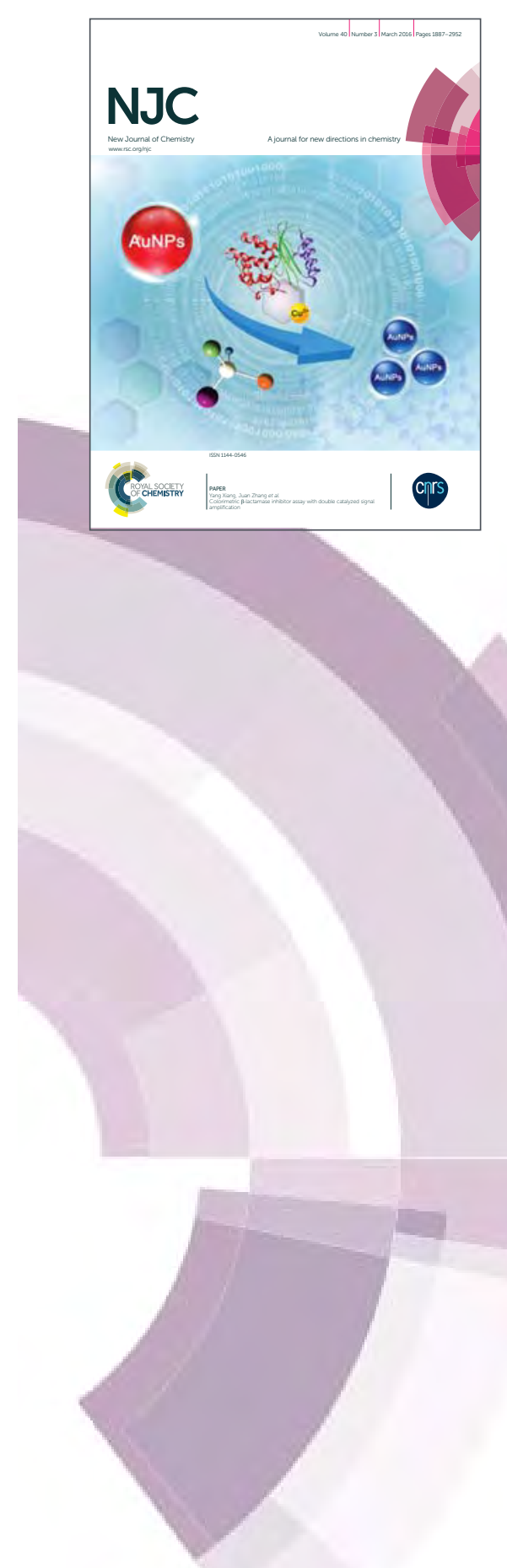

NJC is a general chemistry journal. We solicit innovative and cuttingedge reports of high quality and broad appeal that have the potential to open new directions in chemistry or other scientific disciplines. Both experimental and theoretical works are welcome.

\title{
The following manuscript has been submitted for consideration as a PAPER
}

Papers report a complete study that leads to new understanding or gives new insight into the subject under investigation. If preliminary results have been published in a communication, a subsequent full paper should include additional results that justify another publication.

The Editors and Editorial Board ask you as a reviewer to keep the criteria in mind when making your recommendation for publication in NJC. Routine or incremental work, however competently researched and reported, should not be recommended for publication in $N J C$ as it does not meet our expectations with regard to novelty and impact.

Thank you for your help with the evaluation of this submission. The editors rely on experts such as yourself to improve the scientific quality of the journal. Please support your answers to the questions with appropriate comments to allow the editors to make the best decision and the authors to properly revise their manuscript.

If you recommend Major Revision or Reject and Resubmit then we would appreciate it if you would indicate your willingness to reevaluate the manuscript after revision.

We very much appreciate it if you can respect the deadline for filing your report. If you should need additional time to complete your report, please contact the editors at NJC@rsc.org.

\author{
Professor Mir Wais Hosseini \\ Editor-in-Chief of NJC
}

We also invite you to consider NJC for one of your upcoming manuscripts. Submissions can be made on the Scholar One website: http://mc.manuscriptcentral.com/njc or follow the 'submit an article' link on the NJC homepage given below. 


\title{
Journal Name
}

\section{ARTICLE}

\section{Sensing of Diclofenac by a Porphyrin-based Artificial Receptor}

Received 00th January 20xx, Accepted 00th January 20xx

DOI: $10.1039 / \times 0 \times x 00000 x$

www.rsc.org/

\author{
Daniela Intrieri, ${ }^{\text {C Caterina Damiano, }}{ }^{\text {a }}$ Silvia Rizzato, ${ }^{a}$ Roberto Paolesse, ${ }^{\mathrm{b}}$ Mariano Venanzi, ${ }^{\mathrm{b}}$ Donato \\ Monti, ${ }^{\mathrm{b}}$ Marco Savioli, ${ }^{\mathrm{b}}$ Manuela Stefanelli ${ }^{\mathrm{b}}$ and Emma Gallo. ${ }^{{ }^{\mathrm{a}}}$
}

\begin{abstract}
The reported studies deals with the synthesis of porphyrin chemosensor $\mathbf{2}$, designed for the detection of the emerging pollutant Diclofenac. Owing to the peculiar structure of its molecular frame, which is composed by a tetrapyrrolic platform linked to a Rhodamine B residue, receptor 2 reversibly interacts with Diclofenac sodium salt (DCF)Na. The resulting 2@DCF adduct was detected by UV-Vis spectroscopy in a large $\mathrm{pH}$ range (5.5-9.0) as well as in the presence of competitive analytes. Both static and time-resolved Fluorescence, Resonance Light Scattering (RLS) and UV-Vis spectroscopies allowed for the evaluation of the binding behaviour, in terms of association constant and structural features of formed 2@DCF. In particular, the host-guest recognition event occurs with the growth of large porphyrin aggregates, as stated by the quenching of the fluorescence emission as well as the enhancement of RLS intensities, and with an overall 1:1 binding constant of about $10^{5} \mathrm{M}^{-1}$.
\end{abstract}

\section{Introduction}

Water management has become an urgent worldwide policy issue for the $21^{\text {st }}$ century, consequently the development of efficient and selective procedures for the detection of water pollutants is a topic of global interest and concern. In the last few years a special attention has been given to the identification of Contaminants of Emerging Concern (CECS) in surface water, wastewater as well as groundwater. This class of compounds includes those chemicals that are widely spread in the environment for their abundant use and consequentially they are potentially dangerous for human health. ${ }^{1}$ Examples of CECs are pharmaceuticals and chemicals for personal care and their increasing use can have dramatic outcomes for the ecosystem sustainability. ${ }^{2}$

Among all the emerging contaminants, Diclofenac (Figure 1), an anti-inflammatory drug that was recently inserted into the priority emerging pollutants list by the $\mathrm{EU},{ }^{3,4}$ does not exhibit immediate toxic effects towards living organisms, although a continuing exposure may be responsible for serious environmental damage. Diclofenac is usually detected by employing time-consuming, expensive and off-site classical analytic procedures, mainly chromatographic methods. ${ }^{5-10}$ Although analytical instrumentation cannot be overtaken in terms of sensitivity and selectivity, it cannot allow the on-site

\footnotetext{
a. Department of Chemistry, University of Milan, Via C. Golgi 19, 20133 Milan (Italy). E-mail:emma.gallo@unimi.it.

${ }^{b .}$ Department of Chemical Science and Technologies, University of Roma Tor Vergata, Via della Ricerca Scientifica, 00133 Rome (Italy) E-mail: dmonti@uniroma2.it.

†Electronic supplementary information (ESI) available: experimental details, characterization data, crystallographic data in CIF, supporting tables and crysta data CCDC 1843312. See DOI: 10.1039/x0xx00000x.
}

continuous monitoring, which is an essential requisite for providing prompt remediation procedures. Chemical sensors represent a viable solution to these requirements and sensitive, rapid, cost-effective and real-time analyses are now feasible by employing electrochemical, ${ }^{11-13}$ supramolecular ${ }^{14,15}$ and biomolecular ${ }^{16,17}$ user-friendly sensors. A key component of these devices is the sensing material which is responsible for the molecular recognition (interaction with Diclofenac) and triggering the transduction event (optical, photophysical, electrical, etc.) for sensor signalling.

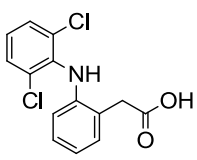

Figure 1. Structure of Diclofenac.

The advancement of the supramolecular chemistry concepts has allowed the rational design of molecular receptors, able to satisfy the sensitivity and selectivity requirements for the binding of target analytes.

Among the different materials that have been exploited as recognising layers, porphyrins have assumed a role of ever-growing importance as responsive materials due to their physico-chemical properties, ${ }^{18-20}$ which allow a significant number of potential transductions to detect even low amounts of the target analyte. The interaction of Diclofenac with the metal (e.g. iron $^{21}$ or manganese $\mathrm{e}^{22}$ ), coordinated into the porphyrin core, often represents the key-point of the sensing mechanism of metal porphyrin-based chemosensors.

We here report the synthesis, characterisation and study of the sensing ability of a porphyrin chemosensor containing Rhodamine $B$, which interacts with Diclofenac without forming covalent bonds, therefore favouring the recycling and reuse of 
the device. Reported data represent the first step for the development of a chemical sensor, which is currently ongoing in our laboratories and will be reported in the due course.

\section{Results and discussion}

Knowing that i) Rhodamine $B(\mathrm{RhB})$ presents a very good affinity for Diclofenac, ${ }^{23,24}$ ii) $\mathrm{RhB}^{25}$ and porphyrin ${ }^{26}$ molecules are very active fluorescent probes and iii) porphyrin-RhB conjugated systems are efficient dyads for electron transfer mechanisms, ${ }^{27-29}$ we synthesised porphyrin $\mathbf{2}$ by using a slight modification of a reported procedure. ${ }^{30}$ Porphyrin 2 was obtained by reacting porphyrin $\mathbf{1}$ with RhB in the presence of $\mathrm{KI}$, which triggered the formation of porphyrin/RhB species as an iodine salt (Scheme 1). The sensing capacity of $\mathbf{2}$ was tested by analysing UV-Vis and Fluorescence spectral patterns in the presence of the target analyte (see below).

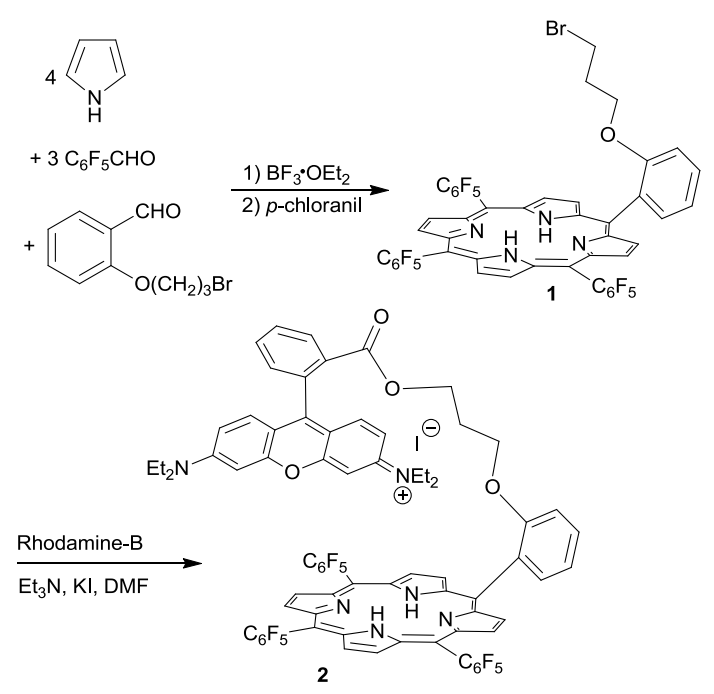

Scheme 1. Synthesis of porphyrin $\mathbf{2}$

Porphyrin 2 was fully characterised, the ${ }^{1} \mathrm{H}$ NMR analysis confirmed the proposed structure and the UV-Vis spectrum showed an intense Soret band at $412 \mathrm{~nm}$ ( $\log \varepsilon M=5.67)$. It should be noted that $Q$ porphyrinic bands are partially superimposed to the intense band at $559 \mathrm{~nm}(\log \varepsilon M=5.24)$ of the RhB moiety, linked to the porphyrin platform. Indeed, the free RhB in methanol shows an intense absorption around $551 \mathrm{~nm}$

It is to note that the meso-pentafluorophenyl substituents of the porphyrin ring were chosen to strengthen the hydrophobic character that, together with the polar hydrophilic iminium salt of RhB, assures the amphiphilic nature of $\mathbf{2}$. This feature will be fundamental for anchoring the molecule onto the solid surface by non-covalent interactions, ${ }^{31,32}$ needed for the future sensor development, and for the interaction with the polar analyte in the water medium, respectively.

Before testing the reactivity of $\mathbf{2}$ towards Diclofenac, its chemical stability was studied in order to evaluate the strength of the RhB/porphyrin linkage. It should be noted that RhB leaching not only would hamper the recyclability of the device but could also be responsible for contamination events. ${ }^{33}$

Although promoting a future anchorage of the device onto the surface, the low hydrophilic character of $\mathbf{2}$ prevented the execution of stability tests in pure water. Thus, to obtain a homogeneous solution, a $\mathrm{H}_{2} \mathrm{O} / \mathrm{CH}_{3} \mathrm{OH}=1: 1$ (v:v) mixture was employed as the solvent. In this polar mixture porphyrin $\mathbf{2}$ was stable for at least $72 \mathrm{~h}$, as revealed by UV-Vis spectroscopic analyses. Good stability was also maintained by changing the $\mathrm{pH}$ of the medium. Several UV-Vis spectra were performed in the 6.5-9.5 pH range and collected data indicated that 2 decomposed only when stirred under strong basic conditions $(\mathrm{pH}>9)$ for more than 24 hours. It is important to underline that in a real-time analysis, the sensor is exposed to the polluted water only for a short period of time, during which the chemical stability in both acidic and basic media is assured. In view of positive stability tests, the chemical interaction between $\mathbf{2}$ and the sodium salt of Diclofenac, (DCF) Na, which is the active principle of Diclofenac-based pharmaceuticals, was investigated in $\mathrm{H}_{2} \mathrm{O} / \mathrm{CH}_{3} \mathrm{OH}=1: 1$ (v:v) mixture (Figure 2).
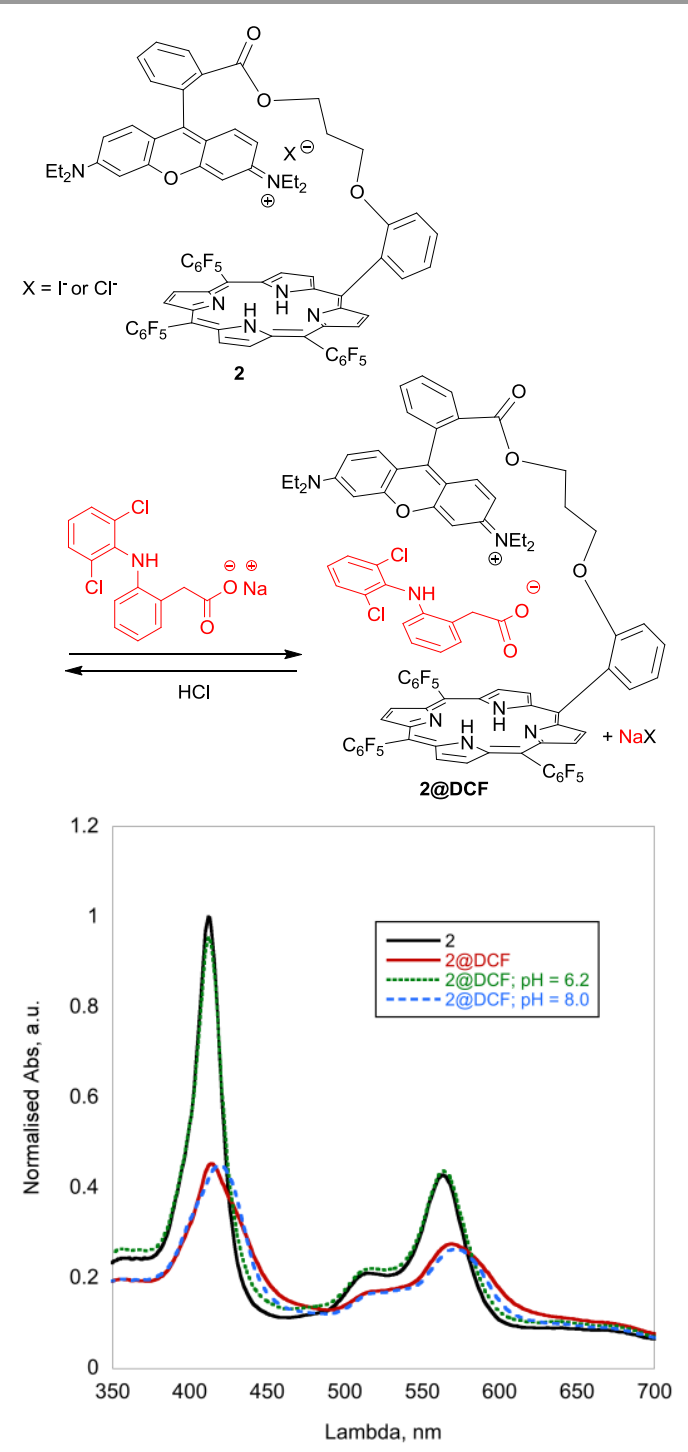

Figure 2. UV-Vis spectra showing the reversible formation of 2@DCF adduct. 
The treatment of porphyrin 2 with (DCF)Na resulted in evident UV-Vis spectral changes, indicating the formation of 2@DCF adduct. The UV-Vis spectrum of $2\left(1.25 \times 10^{-5} \mathrm{M}\right)$ (black line) dropped in intensity and showed a bathochromic effect (red line) after the addition of (DCF)Na in a 3:1 ratio with respect to 2 (Figure 2). It should be noted that the successive addition of $\mathrm{HCl}\left(1,0 \mathrm{~mL}, 1.3 \times 10^{-2} \mathrm{M}\right)$ to reach $\mathrm{pH}=6.2$ was responsible for the regeneration of $\mathbf{2}$ (dotted green line) as chloride salt and Diclofenac as a neutral species by protonation of the carboxylate group. It is worthwhile to note that, on changing the $\mathrm{pH}$ of the mixture from 6.2 to 8.0 by adding a $\mathrm{NaOH}$ solution, 2@DCF adduct was restored (dotted blue line) indicating a complete reversibility of the sensing mechanism (see ESI for experimental details).

Even if the formation of 2@DCF was easily observed by UV-Vis spectroscopy, all the attempts to isolate the adduct as a pure solid failed to suggest the onset of weak interactions between $\mathbf{2}$ and Diclofenac. This result was in agreement with the good reversibility of the sensing process which is an important feature in preventing the chemosensor deactivation and assuring the reversibility needed for the development of a sensors device.

Then, in order to confirm the essential role of $\mathrm{RhB}$ in the sensing process, we synthesised porphyrin $\mathbf{3}$ (Scheme 2), which displays an ammonium salt in place of Rhodamine salt, and its sensing ability towards Diclofenac was investigated. Porphyrin 3 was fully characterised and the UV-Vis spectrum in methanol of 3 showed an intense Soret band at $409 \mathrm{~nm}(\log \varepsilon \mathrm{M}=5.49)$ and four weaker $Q$ bands at $505 \mathrm{~nm}$ (4.27), $535 \mathrm{~nm}$ (3.39), 580 $\mathrm{nm}(3.76)$ and $635 \mathrm{~nm}$ (2.96) (ESI).

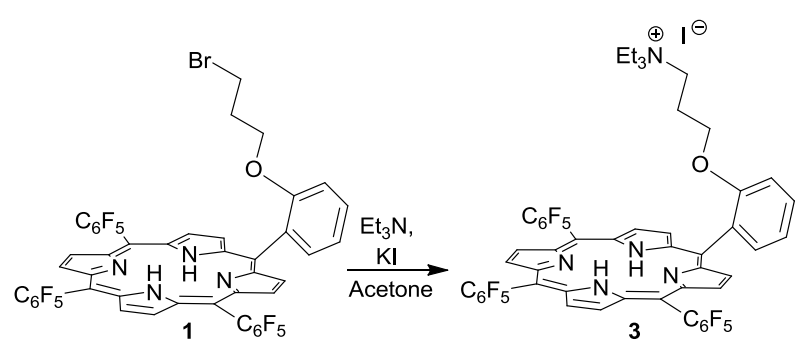

Scheme 2. Synthesis of porphyrin $\mathbf{3}$.

The molecular structure of $\mathbf{3}$, determined by single crysta X-ray diffraction, showed a "close" conformation (Figure 3). The amino-alkyl side arm is folded toward the core of the macrocycle with the terminal methyl groups of the pendant triethylammonium moiety pointing toward the pyrrole rings. Despite the asymmetric arrangement of the substituents, all the conformational data (see ESI for crystallographic details) seem indicating very small distortions of the macrocycle and a square-like inner cavity shape. This three-dimensional arrangement is very interesting in view of the desired synergic activity of the tetrapyrrolic core with the recognition antenna in forming a 'binding pocket' for the analyte.

Porphyrin 3 was treated with (DCF)Na by using the same experimental conditions that were employed for the reaction between porphyrin $\mathbf{2}$ and (DCF)Na. The UV-Vis analysis did not reveal any interaction between $\mathbf{3}$ and the analyte, confirming that RhB was crucial for the sensing recognition and the sole presence of the ammonium salt was not sufficient to interact with the sodium salt of Diclofenac by a metathesis reaction.

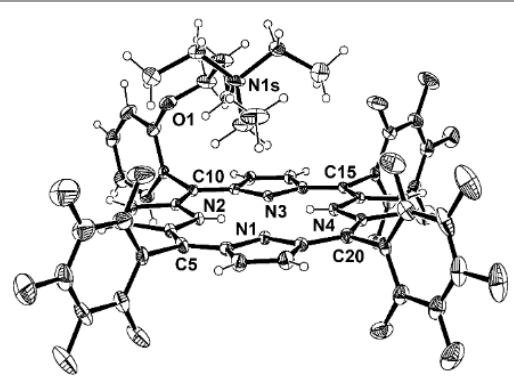

Figure 3. ORTEP diagram of the porphyrin 3. Thermal ellipsoids are shown at $30 \%$ probability levels. The iodine counterion and hexane solvent molecules are omitted for clarity.

Then, in order to evaluate a possible recognition role of $\mathrm{NH}$ functionalities of the tetrapyrrolic core, which could interact with the carboxylate group of Diclofenac by forming hydrogen bonds, the free porphyrin 2 was reacted with $\mathrm{Zn}(\mathrm{OAc})_{2} \cdot\left(\mathrm{H}_{2} \mathrm{O}\right)_{2}$ to yield $\mathrm{Zn(2)}$ complex. The latter complex was fully characterised and showed the same chemical stability of its precursor $\mathbf{2}$ in a polar medium at different $\mathrm{pH}$ values (see above). The study of the sensing activity of $\mathbf{Z n ( 2 )}$ was evaluated and compared with that of the free base porphyrin 2. Collected data indicated that the two compounds showed similar sensing capabilities, suggesting that neither the inner $\mathrm{NH}$ functionalities nor the coordinated $\mathrm{Zn}$ (II) ion are directly involved in the molecular recognition event.

This hypothesis was confirmed by the inertness toward (DCF) Na of the analogue $\mathrm{Zn}$ (TPP) (TPP = dianion of tetraphenyl porphyrin), which does not present any functional group on the molecular skeleton. In fact, the UV-Vis analysis of the reaction of $\mathrm{Zn}(\mathrm{TPP})$ with a stoichiometric excess of (DCF)Na $(\mathrm{Zn}(\mathrm{TPP}) /(\mathrm{DCF}) \mathrm{Na}=1: 5)$ did not reveal any change in the spectrum of the macrocycle indicating that either $\mathrm{Zn}$ (TPP) did not interact with the analyte or, if an interaction exists, this is below the detection limits of UV-Vis spectroscopy. This study suggested that the Diclofenac detection by $\mathbf{2}$ is dictated by the presence of Rhodamine group in the chemosensor periphery. Thus, in order to better assess the role of the porphyrin fragment in the sensing mechanism, the reaction between free Rhodamine $B$ and (DCF)Na was analysed by UV-Vis spectroscopy. No change of the spectral pattern of the substrates was observed suggesting that the sensing performance of $\mathbf{2}$ must be assigned to the entire molecule and not only to the Rhodamine unit. All data reported up to now indicate that the recognition process seems to occur by a lock and key mechanism due to a specific three-dimensional arrangement which is driven by a combination of electrostatic and $\pi-\pi$ interactions between the analyte and receptor.

The evaluation of the binding constant value for the formation of 2@DCF molecular complex was carried out by UV-Vis spectroscopy (Figure 4). In order to keep the concentration of the receptor at a constant value throughout the titration, the (DCF)Na solution was prepared by dissolving the required 
amount of salt into a $10.0 \mu \mathrm{M}$ porphyrin solution itself. Experimental data were conveniently fitted by an overall 1:1 binding equilibrium; a Langmuir type equation revealed a good efficiency of the binding with the experimental $K_{\text {bind }}$ value of $9.3( \pm 0.8) \times 10^{4} \mathrm{M}^{-1}$. Experiments were performed twice and the obtained results showed a very good reproducibility (see ESI for experimental details).

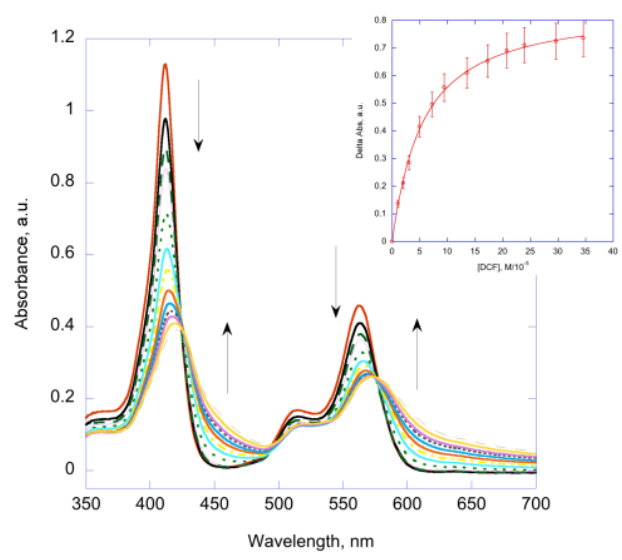

Figure 4. Spectral pattern variation of $2\left(5.0 \mu \mathrm{M} ; \mathrm{MeOH} / \mathrm{H}_{2} \mathrm{O}=1: 1\right)$. Inset: evaluation of the binding constant value for the $2 @ D C F$ formation by a Langmuir type equation.

It should be noted that when $\mathbf{2}$ was left standing in a solid state for a prolonged time (some weeks), some extent of aggregation occurred, as stated by the typical UV-Vis spectral changes (hypochromicity and broadening of the porphyrin Soret B band). However, this process did not affect the binding properties of $\mathbf{2}$, as supported by the reproducibility of $K_{\text {bind }}$ values (within the experimental errors) which were calculated by using samples of $\mathbf{2}$ after different periods of storage.

Importantly, similar $K_{\text {bind }}$ values were obtained by analysing the formation of Zn(2)@DCF adduct to definitely rule out an active role of the central zinc metal during the molecular recognition event.

A closer inspection of the spectroscopic variations, due to the chemosensor/analyte interaction, gave more insights on the intimate sensing mechanism. The molecular binding occurred with the concomitant formation of aggregated structures, which is driven by the saturation of the electronic positive charges of $\mathbf{2}$. The aggregation process was revealed by the strong hypochromic and bathochromic shift of the 2@DCF spectral features upon the Diclofenac addition (Figure 4) and by the concomitant increase of the Resonance Light Scattering (RLS) signal of the adduct (Figure 5). It is worth noting that RLS, i.e. the enhancement of the scattered light close to the absorption wavelength, is shown to be a sensitive and selective method for studying electronically coupled chromophore arrays, such as porphyrins and related macrocycles, due to the great dependence of the signal intensity upon the extent of arrays. ${ }^{37}$

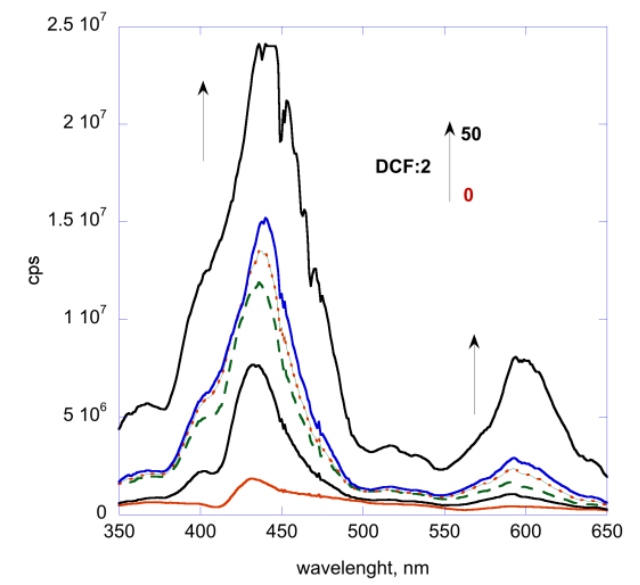

Figure 5. Resonance Light Scattering spectral variation of $2(5.0 \mu \mathrm{M})$ upon the (DCF)Na addition.

The inspection of Figure 5 revealed a strong increase of the scattered signal upon the (DCF)Na addition. This, jointly with the corresponding variation observed in the UV-Vis spectra, strongly indicated the formation of large arrays of porphyrin aggregates, up to a micrometric scale.

Analogous studies were also being carried out by using Fluorescence Spectroscopy. Fluorescence emission spectra of $\mathbf{2}$ were measured in a $\mathrm{H}_{2} \mathrm{O} / \mathrm{CH}_{3} \mathrm{OH}=1: 1(\mathrm{v}: \mathrm{v})$ solvent mixture by exciting the dyad compound at $\lambda_{\text {exc }}=412 \mathrm{~nm}$. Three emission peaks were detected at $\lambda_{\mathrm{em}}=590,641$ and $705 \mathrm{~nm}$, which were attributed to Rhodamine $B$ and porphyrin moieties, respectively. These values shifted to longer wavelengths with respect to the emission maxima of $3\left(\lambda_{\mathrm{em}}=637\right.$ and $\left.699 \mathrm{~nm}\right)$ and Rhodamine $B$ alone $\left(\lambda_{\text {em }}=569 \mathrm{~nm}\right)$ suggesting an electronic coupling of the fluorophores in the dyad compound. Interestingly, the collection of the excitation spectra of $\mathbf{2}$ at $\lambda_{\mathrm{em}}=590 \mathrm{~nm}$ (RhB emission) showed a distinct contribution from the porphyrin absorption spectrum. Analogously, a relevant contribution from the RhB absorption spectrum was detected by collecting the excitation spectra of $\mathbf{2}$ at $\lambda_{\text {em }}=705$ (porphyrin emission) (See ESI). These findings clearly indicated that excited state Energy Transfer (ET) processes took place from the Rhodamine excited state to the tetrapyrrolic moiety and vice versa from the porphyrin excited state to the Rhodamine group. Due to the observed ground state electronic coupling between the two fluorophores, ET can be safely ascribed to a Dexter mechanism, which requires a short-range interaction between the electronic distributions of the two components of the dyad. These results confirmed that the receptor $\mathbf{2}$ preferentially attains a molecular conformation in which the two chromophores are located at short distances apart.

The DCF/ 2 binding interactions were also investigated by measuring the emission spectra of $\mathbf{2}$ at different (DCF)Na concentrations by using the same experimental conditions which were employed for UV-Vis absorption experiments.

Achieved data indicated a strong quenching of the $\mathbf{2}$ emission for both the excitation wavelengths related to Rhodamine and porphyrin groups. This effect can be ascribed to either i) a 
direct quenching of Diclofenac on both the chromophores or ii) the breaking of the Rhodamine-porphyrin excited state interaction due to a decrease of the electronic coupling between the two chromophores. However, both mechanisms require that Diclofenac should be placed at very short distances with respect to the two chromophores, likely intercalated between the porphyrin and Rhodamine moieties (Figure 2).

The 2@DCF binding isotherm, obtained by measuring the fluorescence intensities of the porphyrin moiety at different (DCF) Na concentrations, displayed the $K_{\text {bind }}$ of $2.7( \pm 0.3) \times 10^{4} \mathrm{M}^{-1}(\mathrm{ESI})$ which is in fair agreement with the $K$ value calculated by using UV-Vis spectroscopy. The small discrepancy between the two $K$ values $\left(9.3( \pm 0.8) \times 10^{4} \mathrm{M}^{-1}\right.$ by UV-Vis absorption versus $2.7( \pm 0.3) \times 10^{4} \mathrm{M}^{-1}$ by fluorescence emission) could be ascribed to the fact that UV-Vis spectroscopy only depends on ground-state interactions while fluorescence is affected by the extent of both ground- and excited state interactions. Time-resolved fluorescence experiments, carried out on the porphyrin emission at different (DCF)Na concentrations, clearly demonstrated the onset of excited state interactions between the porphyrin moiety and DCF. In particular, the analysis showed, upon the DCF binding, a marked quenching of all the three lifetimes accounting for the time decay of the porphyrin moiety. This finding indicates that DCF binding affected both monomeric ( $\tau=11 \mathrm{~ns}$ ), and aggregated ( $\tau=2-1 \mathrm{~ns}$ ) forms of 2 , with the last species becoming the most abundant in the presence of a (DCF) Na excess (ESI).

In order to verify the sensor selectivity, the reactivity of both 2 and $\mathbf{Z n ( 2 )}$ species towards sodium salt of Diclofenac was investigated in the presence of $\mathrm{Pb}^{2+}$ or $\mathrm{Cd}^{2+}$ metal pollutants as well as ammonia $\left(1.28 \times 10^{-4} \mathrm{M}\right.$, in a $1: 1$ molar ratio with respect to Diclofenac). UV-Vis spectroscopic studies revealed that the sensing ability of both porphyrin chemosensors was not affected by the presence of the above listed species, underlining the good selectivity of these receptors towards the target analyte. Quantitative binding experiments have been carried out in the presence of possible interfering carboxylate drugs such as sodium phenylacetate, sodium salicylate, L-tyrosine sodium salt, sodium sarcosinate and L-alanine sodium salt. The results obtained showed that the binding ability of receptor $\mathbf{2}$ was very slightly affected by the presence of the above contaminants at both $1: 1$ and 1:5 molar ratio, with respect to the DCF concentration (Figure 6, see ESI for experimental details).

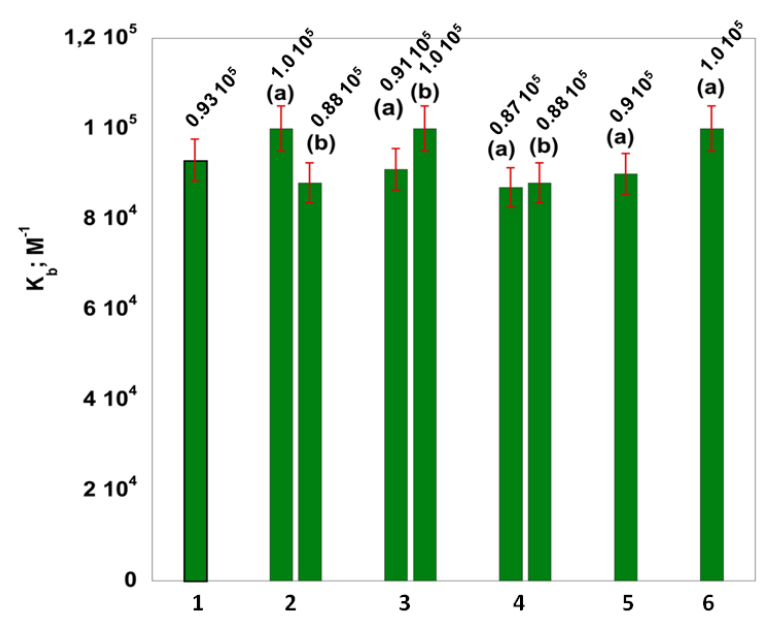

$1=(D C F) N a ; 2$ = sodium phenylacetate; 3 = sodium salicylate;

4 = L-tyrosine sodium salt; $\mathbf{5}=$ sodium sarcosinate; $6=\mathrm{L}$-alanine sodium salt

(a): $($ DCF) Na/competitor $=1: 1$; (b): $($ DCF) Na/competitor $=1: 5$

Figure 6. Binding constant values for the formation of $2 @ D C F$ adduct in the presence of interfering sodium salts. (a): 1:1 molar ratio, (b): 1:5 molar ratio (error bars 5\%).

\section{Conclusions}

In conclusion, we reported the synthesis and full characterisation of the porphyrin/Rhodamine B molecular chemosensor $\mathbf{2}$ and its zinc complex $\mathbf{Z n ( 2 )}$ which were tested for the Diclofenac detection. UV-Vis spectroscopic analyses of the recognition reactions revealed a reversible sensing process which occurred by a lock and key mechanism in which the binding of the analyte was the result of the synergic interaction of the analyte with both the Rhodamine unit (electrostatic interactions) and the tetrapyrrolic platform ( $\pi-\pi$ and London dispersion forces). The binding of the analyte was mainly due to the Rhodamine unit and the tetrapyrrolic core only favoured the Diclofenac accommodation without forming specific bonds.

Future studies will be devoted to the exploitation of the investigated receptor as sensing material for the development of a chemical sensor. For this application, we will investigate the introduction of a spacer group on the para position of meso-aryl groups in order to anchor the chemosensor onto a surface by forming either covalent or non-covalent linkages.

\section{Conflicts of interest}

There are no conflicts to declare.

\section{Notes and references}

1 S. D. Richardson and T. A. Ternes, Anal. Chem., 2018, 90 398-428.

2 T. Deblonde, C. Cossu-Leguille and P. Hartemann, Int. J. Hyg. Environ. Health, 2011, 214, 442-448.

3 J. C. G. Sousa, A. R. Ribeiro, M. O. Barbosa, M. F. R. Pereira and A. M. T. Silva, J. Hazard. Mater., 2018, 344, 146-162.

4 Directive 2013/39/UE of the European Parliament and the Council of the 12 amending Directive 2000/60/EC and 
2005/105/EC regards priority substances in the field of water policy, Official J. Eur. Union, 2013.

5 S. Schmidt, H. Hoffmann, L.-A. Garbe and R. J. Schneider, J. Chromatogr. A, 2018, 1538, 112-116.

6 G. Daniele, M. Fieu, S. Joachim, A. Bado-Nilles, P. Baudoin, C. Turies, J.-M. Porcher, S. Andres and E. Vulliet, Anal. Bioanal. Chem., 2016, 408, 4435-4444.

7 P. C. Lindholm-Lehto, H. S. J. Ahkola, J. S. Knuutinen, J. Koistinen, K. Lahti, H. Vahtera and S. H. Herve, Environ. Sci. Pollut. R., 2016, 1-12.

8 V. Osorio, M. Imbert-Bouchard, B. Zonja, J.-L. Abad, S. Pérez and D. Barceló, J. Chromatogr. A, 2014, 1347, 63-71.

9 N. Hermes, K. S. Jewell, A. Wick and T. A. Ternes, J. Chromatogr. A, 2018, 1531, 64-73.

10 C. E. D. Nazario and F. M. Lancas, Food Anal. Method, 2017, 10, 2490-2496.

11 S. Motoc, F. Manea, A. lacob, A. Martinez-Joaristi, J. Gascon, A. Pop and J. Schoonman, Sensors, 2016, 16, 1719-1730.

12 G. P. Fard, E. Alipour and R. E. Ali Sabzi, Anal. Methods, 2016 , 8, 3966-3974.

13 G. Y. Aguilar-Lira, J. M. Gutiérrez-Salgado, A. RojasHernández, J. A. Rodríguez-Ávila, M. E. Páez-Hernández and G. A. Álvarez-Romero, J. Electroanal. Chem., 2017, 801, 527535.

14 F. Ahmed, K. Shah, I. Z. Awan and M. R. Shah, Ecotoxicol. Environ. Saf., 2016, 129, 103-108.

15 K. Shah, E. Hassan, F. Ahmed, I. Anis, M. Rabnawaz and M. R. Shah, Ecotoxicol. Environ. Saf., 2017, 141, 25-29.

16 N. Steinke, M. Rio, R. Wuchrer, C. Schuster, E. Ljasenko, D. Knopp, G. Gerlach and T. Härtling, Sens. Actuators B Chem. 2018, 254, 749-754.

17 J. Shi, M. Xu, Q. Tang, K. Zhao, A. Deng and J. Li, Spectrochim. Acta A Mol. Biomol. Spectrosc., 2018, 191, 1-7.

18 R. Paolesse, S. Nardis, D. Monti, M. Stefanelli and C. Di Natale, Chem. Rev., 2017, 117, 2517-2583.

19 Y. Ding, W.-H. Zhu and Y. Xie, Chem. Rev., 2017, 117, 22032256.

20 N. L. Bill, O. Trukhina, J. L. Sessler and T. Torres, Chem. Commun., 2015, 51, 7781-7794.

21 E. M. G. Santos, A. N. Araújo, C. M. C. M. Couto and M. C. B. S. M. Montenegro, J. Pharm. Biomed. Anal., 2006, 42, 535542.

22 D. Vlascici, S. Pruneanu, L. Olenic, F. Pogacean, V. Ostafe, V. Chiriac, E. M. Pica, L. C. Bolundut, L. Nica and E. FagadarCosma, Sensors, 2010, 10, 8850-8864.

23 G. Pranitha and G. Venkateshwarlu, World J. Pharm. Pharm. Sci., 2016, 5, 2249-2260.

24 Z. Kormosh, I. Hunka, Y. Bazel, A. Laganovsky, I. Mazurenko and N. Kormosh, Cent. Eur. J. Chem., 2007, 5, 813-823.

25 R. Zhang, F. Yan, Y. Huang, D. Kong, Q. Ye, J. Xu and L. Chen, RSC Advances, 2016, 6, 50732-50760.

26 O. Horváth, Z. Valicsek, M. A. Fodor, M. M. Major, M. Imran, G. Grampp and A. Wankmüller, Coord. Chem. Rev., 2016, $325,59-66$

27 E. J. Ngen, L. Xiao, P. Rajaputra, X. Yan and Y. You, Photochem. Photobiol., 2013, 89, 841-848.

28 T. Mani, D. M. Niedzwiedzki and S. A. Vinogradov, J. Phys. Chem. A, 2012, 116, 3598-3610.

29 E. J. Ngen, P. Rajaputra and Y. You, Bioorg. Med. Chem. 2009, 17, 6631-6640.

30 X. Sun, D. Li, G. Chen and J. Zhang, Dyes Pigm., 2006, 71, 118122.

31 H. M. Titi, B. K. Tripuramallu and I. Goldberg, CrystEngComm, 2016, 18, 3318-3339.

32 A. D'Urso, A. Di Mauro, A. Cunsolo, R. Purrello and M. E. Fragalà, J. Phys. Chem. C, 2013, 117, 17659-17665.

33 M. Soylak, Y. E. Unsal, E. Yilmaz and M. Tuzen, Food Chem. Toxicol., 2011, 49, 1796-1799.
34 M. Gruden, S. Grubisic, A. G. Coutsolelos and S. R. Niketic, J. Mol. Struct., 2001, 595, 209-224.

35 W. Jentzen, X.-Z. Song and J. A. Shelnutt, J. Phys. Chem. B, 1997, 101, 1684-1699.

36 W. Jentzen, J.-G. Ma and J. A. Shelnutt, Biophys. J., 1998, 74, 753-763

37 R.F. Pasternack and P.J. Collings, Science, 1995, 269, 935-939. 


\section{Journal Name}

\section{ARTICLE}

\section{Graphical Abstract}

The synthesis of a porphyrin chemosensor is here reported as well as its sensing activity in detecting the emerging pollutant Diclofenac with an overall 1:1 binding constant of about $10^{5} \mathrm{M}^{-1}$.

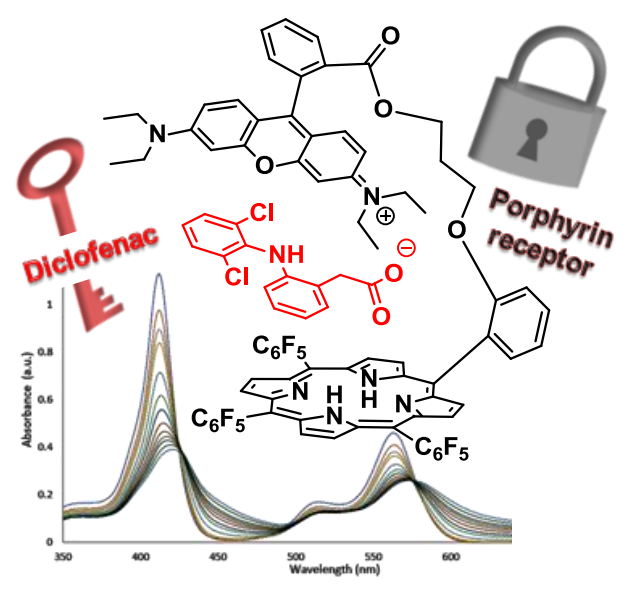


Electronic Supplementary Information

\section{Sensing of Diclofenac by a Porphyrin-based Artificial Receptor}

Daniela Intrieri, ${ }^{\mathrm{a}}$ Caterina Damiano, ${ }^{\mathrm{a}}$ Silvia Rizzato, ${ }^{\mathrm{a}}$ Roberto Paolesse, ${ }^{\mathrm{b}}$ Mariano Venanzi, ${ }^{\mathrm{b}}$ Donato Monti, ${ }^{* \mathrm{~b}}$ Marco Savioli ${ }^{\mathrm{b}}$, Manuela Stefanelli ${ }^{\mathrm{b}}$ and Emma Gallo. ${ }^{* \mathrm{a}}$

${ }^{a}$ Department of Chemistry, University of Milan, Via C. Golgi 19, 20133 Milan (Italy). E-mail: emma.gallo@unimi.it.

${ }^{b}$ Department of Chemical Science and Technologies, University of Roma Tor Vergata, Via della Ricerca Scientifica,00133 Rome (Italy).E-mail:dmonti@uniroma2.it

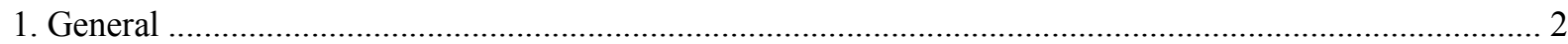

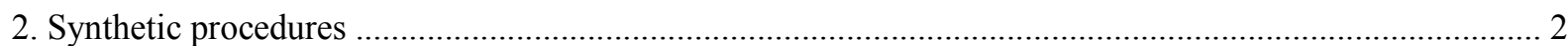

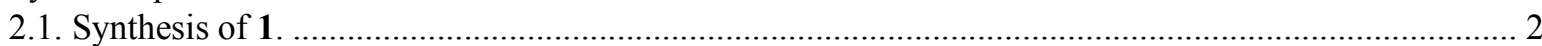

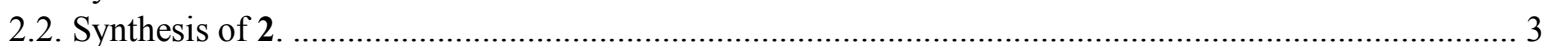

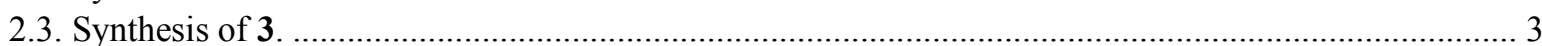

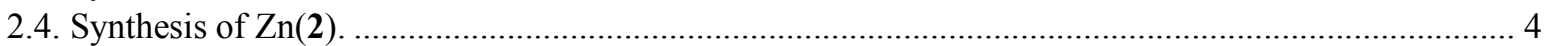

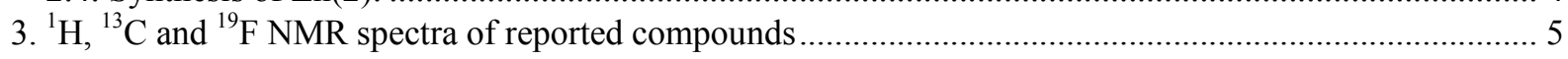

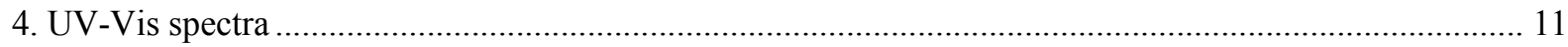

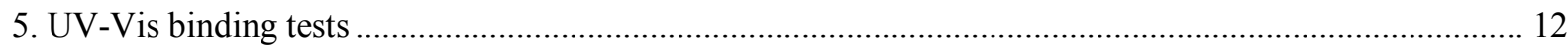

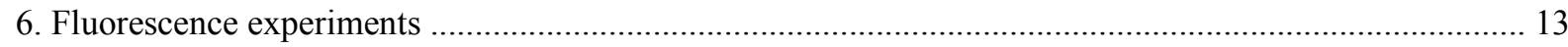

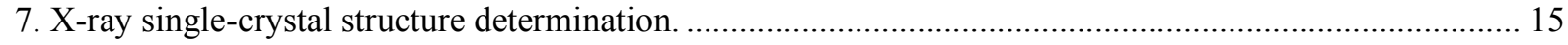

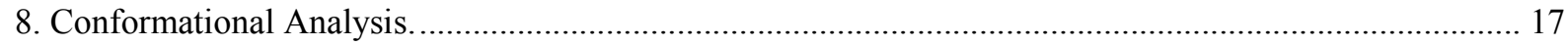

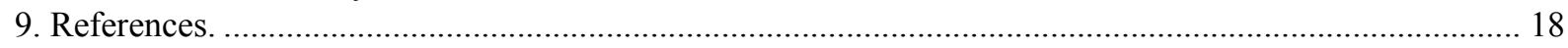




\section{General}

Unless otherwise specified, all the reactions were carried out under a nitrogen atmosphere by employing standard Schlenk techniques and magnetic stirring. Dichloromethane, chloroform, $N, N$-dimethylformamide and pyrrole were distilled over $\mathrm{CaH}_{2}$ and stored under nitrogen. Acetone was distilled over $\mathrm{K}_{2} \mathrm{CO}_{3}$ and stored under nitrogen. All the other starting materials and $\mathrm{Zn}(\mathrm{TPP})$ were commercial products which were used as received. 2-(3-Bromopropoxy)benzaldehyde ${ }^{1}$ and sodium phenylacetate ${ }^{2}$ were synthesized by methods reported in the literature or by using minor modifications of them. Solvents used for spectroscopy investigations were of Spectroscopic Grade of the highest degree of purity available and used as received. NMR spectra were recorded at room temperature on a Bruker Avance 400-DRX spectrometers, operating at $400 \mathrm{MHz}$ for ${ }^{1} \mathrm{H}$, at $100 \mathrm{MHz}$ for ${ }^{13} \mathrm{C}$ and at $376 \mathrm{MHz}$ for ${ }^{19} \mathrm{~F}$. Chemical shifts (ppm) are reported relative to TMS. The ${ }^{1} \mathrm{H}$ NMR signals of the compounds described in the following have been attributed by COSY and NOESY techniques. Assignments of the resonance in ${ }^{13} \mathrm{C}$ NMR were made using the APT pulse sequence and HSQC and HMBC techniques. UV/Vis spectra were recorded on an Agilent 8453E instrument. Elemental analyses and mass spectra were recorded in the analytical laboratories of Milan University. All spectroscopic studies were carried out at $298.0( \pm 0.5) \mathrm{K}$ (Julabo F25 Thermostat). Steady-state Fluorescence and Resonance Light Scattering Spectroscopy studies were carried out on Fluoromax 4 (Horiba Instruments). Time-resolved Fluorescence Spectroscopy studies were carried out on LifeSpoec-ps (Edimburg Instruments), equipped with a Hamamatsu Photonics K.K. laser. X-ray data collection was performed at 150 $\mathrm{K}$ using graphite-monochromated Mo K $\alpha$ radiation $(\lambda=0.71073 \AA)$ on a Bruker ApexII CCD area-detector diffractometer equipped with an Oxford Cryosystems $\mathrm{N}_{2}$ gas blower. A $\omega$-scan was performed within the Bragg limits of $1.3<\theta<25.0^{\circ}$. Determination of the integrated intensities and unit cell refinements were performed using SAINT $^{3}$ and all absorption corrections were applied by using SADABS. ${ }^{4}$ The structures were solved by direct methods (SIR2014) $)^{5}$ and refined by full-matrix least squares on $\mathrm{F}^{2}$ (SHELX 2014) ${ }^{6}$ with the WINGX interface. ${ }^{7}$

\section{Synthetic procedures}

\subsection{Synthesis of 1 .}

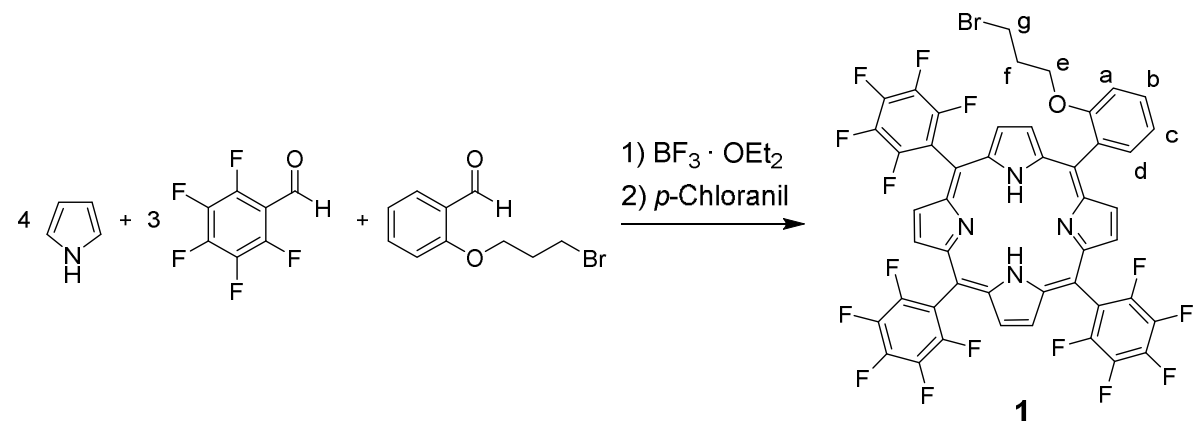

Freshly distilled pyrrole $(173 \mu \mathrm{L}, 2.50 \mathrm{mmol})$, pentafluorobenzaldehyde $(231 \mu \mathrm{L}, 1.87 \mathrm{mmol})$ and 2-(3bromopropoxy)benzaldehyde $(110 \mu \mathrm{L}, 0.62 \mathrm{mmol})$ were dissolved in dry dichloromethane $(250 \mathrm{~mL})$ in a 500 $\mathrm{mL}$ two necks round-bottom flask. The reaction mixture was shielded from ambient light and $\mathrm{BF}_{3} \cdot \mathrm{OEt}_{2}(31$ $\mu \mathrm{L}, 0.25 \mathrm{mmol}$ ) was added dropwise by a syringe. The obtained pale orange solution was stirred in the dark for 3 hours at room temperature and then tetrachloro-1,4-benzoquinone ( $p$-chloranil) $(0.615 \mathrm{~g}, 2.50 \mathrm{mmol})$ was added. The resulting solution was refluxed in air for 6 hours, the solvent evaporated to dryness under reduced pressure and the resulting black solid purified by flash column chromatography on silica gel ( $60 \mu \mathrm{m}$, eluent $n$-hexane/dichloromethane $=90: 10)$ yielding $1(20 \%$ yield $)$ as a purple solid. Elemental Analysis calc. for $\mathrm{C}_{47} \mathrm{H}_{20} \mathrm{BrF}_{15} \mathrm{~N}_{4} \mathrm{O}$ : C, 55.26; H, 1.97; N, 5.48; found: C, 55.66; H, 2.11; N, 5.31. UV-Vis, $\lambda_{\max }(\mathrm{MeOH}) / \mathrm{nm}$ $\left(\log \varepsilon_{\mathrm{M}}\right): 410$ (5.39), 506 (4.47), 536 (3.61), 582 (3.98), 643 (3.15). LR-MS (ESI): $\mathrm{m} / \mathrm{z}\left(\mathrm{C}_{47} \mathrm{H}_{20} \mathrm{BrF}_{15} \mathrm{~N}_{4} \mathrm{O}\right.$ $\left.[\mathrm{M}+\mathrm{H}]^{+}\right)$calcd. 1020.06; found 1021.2. ${ }^{1} \mathrm{H} \mathrm{NMR}\left(400 \mathrm{MHz}, \mathrm{CDCl}_{3}, 298 \mathrm{~K}\right) \delta: 8.97(\mathrm{~d}, J=4.7 \mathrm{~Hz}, 2 \mathrm{H}$, $\left.\mathrm{H}_{\text {ppyrr }}\right), 8.91\left(\mathrm{~s}, 4 \mathrm{H}, \mathrm{H}_{\beta p y r r}\right), 8.84\left(\mathrm{~d}, J=4.7 \mathrm{~Hz}, 2 \mathrm{H}, \mathrm{H}_{\text {ppyrr }}\right), 8.08\left(\mathrm{dd}, J_{o}=7.4 \mathrm{~Hz}, J_{m}=1.5 \mathrm{~Hz}, 1 \mathrm{H}, \mathrm{H}_{\mathrm{d}}\right), 7.83$ $\left(\mathrm{t}, J=8.1 \mathrm{~Hz}, 1 \mathrm{H}, \mathrm{H}_{\mathrm{b}}\right), 7.44\left(\mathrm{t}, J=7.6 \mathrm{~Hz}, 1 \mathrm{H}, \mathrm{H}_{\mathrm{c}}\right), 7.38\left(\mathrm{~d}, J=8.1 \mathrm{~Hz}, 1 \mathrm{H}, \mathrm{H}_{\mathrm{a}}\right), 4.10\left(\mathrm{t}, J=5.4 \mathrm{~Hz}, 2 \mathrm{H}, \mathrm{H}_{\mathrm{e}}\right)$, 
$2.26\left(\mathrm{t}, J=6.0 \mathrm{~Hz}, 2 \mathrm{H}, \mathrm{H}_{\mathrm{g}}\right), 1.46\left(\mathrm{~m}, 2 \mathrm{H}, \mathrm{H}_{\mathrm{f}}\right),-2.81\left(\mathrm{~s}, 2 \mathrm{H}, \mathrm{N} H_{p y r r}\right) .{ }^{13} \mathrm{C} \mathrm{NMR}\left(100 \mathrm{MHz}, \mathrm{CDCl}_{3}, 298 \mathrm{~K}\right) \delta$ : 158.3, 147.8, 145.4, 143.4, 140.9, 138.8, 136.3, 135.6, 130.6, 129.8 (2 signals overlapped), 119.9, 119.4, $116.0,117.7,102.8,101.9,65.1,31.3,29.8$. Six quaternary carbon atoms were not detected. ${ }^{19} \mathrm{~F}$ NMR $(376$ $\left.\mathrm{MHz}, \mathrm{CDCl}_{3}, 298 \mathrm{~K}\right) \delta:-136.9,-152.1,-162.0$.

\subsection{Synthesis of 2 .}
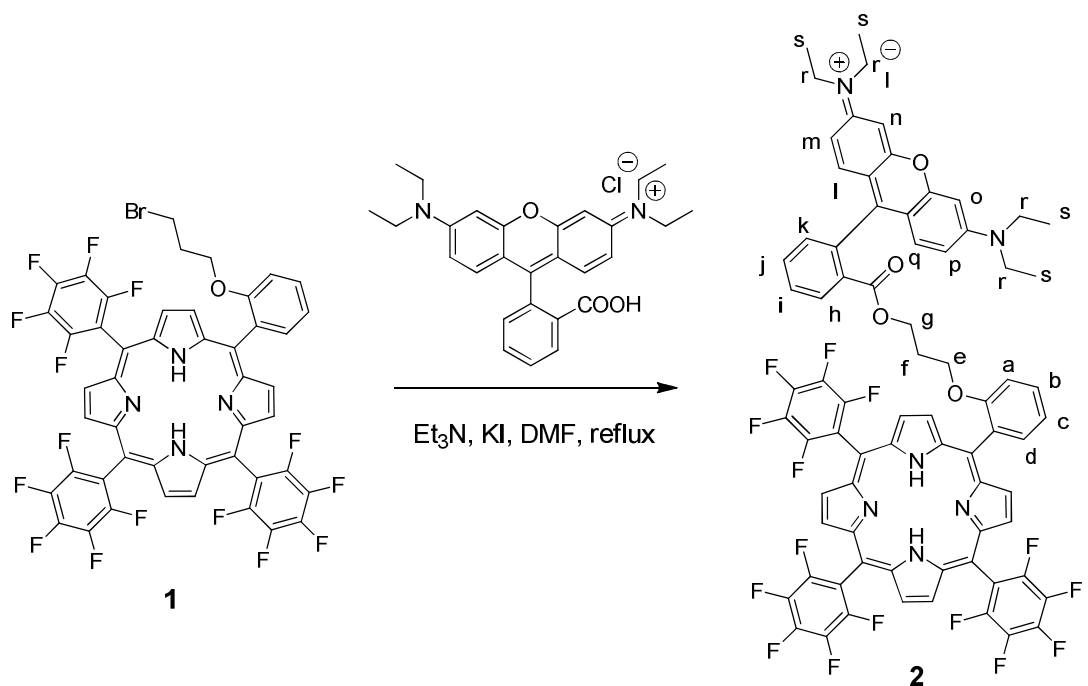

Rhodamine B $(0.200 \mathrm{~g}, 0.418 \mathrm{mmol})$, triethylamine $(58.0 \mu \mathrm{L}, 0.418 \mathrm{mmol})$ and $\mathrm{KI}(0.069 \mathrm{~g}, 0.418 \mathrm{mmol})$ were added to a dry DMF $(25 \mathrm{~mL})$ solution of $1(0.171 \mathrm{~g}, 0.167 \mathrm{mmol})$. The dark purple mixture was refluxed under stirring for 10 hours, then the solvent was evaporated to dryness under reduced pressure and the residue purified by flash column chromatography on silica gel $(60 \mu \mathrm{m}$, eluent: gradient, from dichloromethane to $3 \%$ methanol in dichloromethane) yielding 2 (45\% yield) as a dark purple solid (which resulted pink in solution). Elemental Analysis calc. for $\mathrm{C}_{75} \mathrm{H}_{50} \mathrm{~F}_{15} \mathrm{IN}_{6} \mathrm{O}_{4}: \mathrm{C}, 59.61 ; \mathrm{H}, 3.34 ; \mathrm{N}, 5.56$; found: $\mathrm{C}$, 59.93; H, 3.13; N, 5.83. UV-Vis, $\lambda_{\max }(\mathrm{MeOH}) / \mathrm{nm}\left(\log \varepsilon_{\mathrm{M}}\right): 412$ (5.67), 512 (4.82), 559 (5.24), 638 (3.18). LR-MS (ESI): $\mathrm{m} / \mathrm{z}\left(\mathrm{C}_{75} \mathrm{H}_{50} \mathrm{~F}_{15} \mathrm{~N}_{6} \mathrm{O}_{4}{ }^{+}[\mathrm{M}]^{+}\right)$calcd. 1383.36; found 1383.6. ${ }^{1} \mathrm{H} \mathrm{NMR}\left(400 \mathrm{MHz}, \mathrm{CDCl}_{3}, 298 \mathrm{~K}\right)$ $\delta: 8.87\left(\mathrm{~m}, 6 \mathrm{H}, \mathrm{H}_{\beta p y r r}\right), 8.73\left(\mathrm{~d}, J=4.3 \mathrm{~Hz}, 2 \mathrm{H}, \mathrm{H}_{\beta p y r r}\right), 8.00\left(\mathrm{dd}, J_{o}=7.4 \mathrm{~Hz}, J_{m}=1.6 \mathrm{~Hz}, 1 \mathrm{H}, \mathrm{H}_{\mathrm{d}}\right), 7.78(\mathrm{~m}$, $2 \mathrm{H}, \mathrm{H}_{\mathrm{b}}$ and $\left.\mathrm{H}_{\mathrm{h}}\right), 7.63\left(\mathrm{~m}, 1 \mathrm{H}, \mathrm{H}_{\mathrm{j}}\right), 7.40\left(\mathrm{t}, J=7.4 \mathrm{~Hz}, 2 \mathrm{H}, \mathrm{H}_{\mathrm{c}}\right.$ and $\left.\mathrm{H}_{\mathrm{i}}\right), 7.31\left(\mathrm{~m}, 1 \mathrm{H}, \mathrm{H}_{\mathrm{a}}\right), 7.14\left(\mathrm{~m}, 1 \mathrm{H}, \mathrm{H}_{\mathrm{k}}\right)$, $6.83\left(\mathrm{~d}, J=9.9 \mathrm{~Hz}, 2 \mathrm{H}, \mathrm{H}_{\mathrm{l}}\right.$ and $\left.\mathrm{H}_{\mathrm{q}}\right), 6.64\left(\mathrm{~m}, 4 \mathrm{H}, \mathrm{H}_{\mathrm{m}}, \mathrm{H}_{\mathrm{n}}, \mathrm{H}_{\mathrm{o}}\right.$ and $\left.\mathrm{H}_{\mathrm{p}}\right), 3.87\left(\mathrm{t}, J=5.5 \mathrm{~Hz}, 2 \mathrm{H}, \mathrm{H}_{\mathrm{e}}\right), 3.43(\mathrm{~m}$, $\left.8 \mathrm{H}, \mathrm{H}_{\mathrm{r}}\right), 3.17\left(\mathrm{t}, J=6.1 \mathrm{~Hz}, 2 \mathrm{H}, \mathrm{H}_{\mathrm{g}}\right), 1.13\left(\mathrm{~m}, 14 \mathrm{H}, \mathrm{H}_{\mathrm{f}}\right.$ and $\left.\mathrm{H}_{\mathrm{s}}\right),-2.84\left(\mathrm{~s}, 2 \mathrm{H}, \mathrm{N} H_{p y r r}\right) \cdot{ }^{13} \mathrm{C} \mathrm{NMR}(100 \mathrm{MHz}$, $\left.\mathrm{CDCl}_{3}, 298 \mathrm{~K}\right) \delta: 164.4,158.6,158.1,157.5,155.3,147.8,145.3,143.4,140.9,138.8,135.8,133.2,132.9$, $131.3,131.1,131.0,130.9,130.1,129.62,129.56,120.0,119.4,116.3,114.2,113.3,112.0,102.7,96.3,64.6$, 61.9, 46.1, 43.5, 41.3, 36.0, 29.1 27.8, 12.5. ${ }^{19} \mathrm{~F}$ NMR (376 MHz, $\left.\mathrm{CDCl}_{3}, 298 \mathrm{~K}\right) \delta:-137.0,-140.38,-140.89$, $-152.0,-161.8$.

\subsection{Synthesis of 3 .}
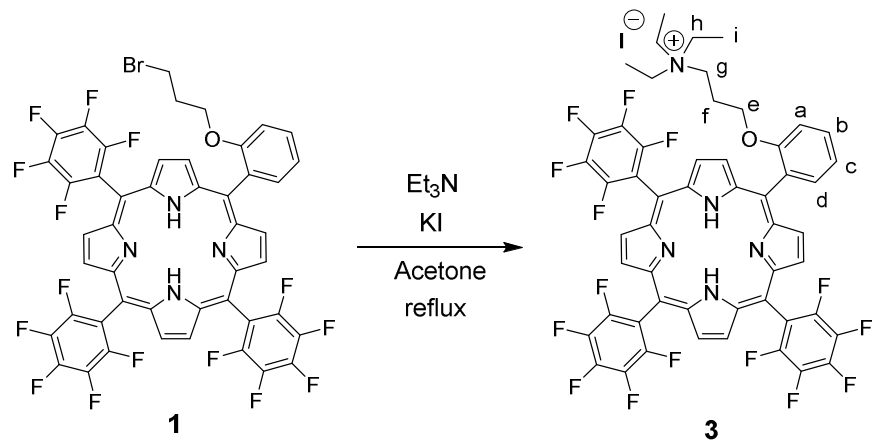

Triethylamine $(0.172 \mathrm{~mL}, 1.24 \mathrm{mmol})$ and $\mathrm{KI}(0.207 \mathrm{~g}, 1.24 \mathrm{mmol})$ were added to a dry acetone $(15 \mathrm{~mL})$ solution of 1 ( $0.127 \mathrm{~g}, 0.124 \mathrm{mmol})$. The dark brown mixture was refluxed under stirring for 6 hours, then 
the solvent evaporated to dryness under reduced pressure and the resulting solid purified by flash column chromatography on silica gel $(60 \mu \mathrm{m}$, eluent: gradient, from dichloromethane to $3 \%$ methanol in dichloromethane) yielding 3 (55\% yield) as a dark red solid. Elemental Analysis calc. for $\mathrm{C}_{53} \mathrm{H}_{35} \mathrm{~F}_{15} \mathrm{IN}_{5} \mathrm{O}$ : C, 54.42; H, 3.02; N, 5.99; found: C, 54.23; H, 3.11; N, 6.06. UV-Vis, $\lambda_{\max }\left(\mathrm{CH}_{2} \mathrm{Cl}_{2}\right) / \mathrm{nm}\left(\log \varepsilon_{\mathrm{M}}\right): 413(5.49)$, 507 (4.28), 536 (3.47), 583 (3.80), 636 (3.14). $\lambda_{\max }(\mathrm{MeOH}) / \mathrm{nm}\left(\log \varepsilon_{\mathrm{M}}\right): 409$ (5.49), 505 (4.27), 535 (3.39), 580 (3.76), 635 (2.96). LR-MS (ESI): $\mathrm{m} / \mathrm{z}\left(\mathrm{C}_{53} \mathrm{H}_{35} \mathrm{~F}_{15} \mathrm{~N}_{5} \mathrm{O}^{+}[\mathrm{M}]^{+}\right)$calcd. 1042.26; found 1042.4. ${ }^{1} \mathrm{H}$ NMR $\left(400 \mathrm{MHz}, \mathrm{CDCl}_{3}, 298 \mathrm{~K}\right) \delta: 8.96\left(\mathrm{~m},, 6 \mathrm{H}, \mathrm{H}_{\text {ppyrr }}\right), 8.89\left(\mathrm{~d}, J=4.5 \mathrm{~Hz}, 2 \mathrm{H}, \mathrm{H}_{\text {ppyrr }}\right), 8.22\left(\mathrm{dd}, J_{o}=7.6 \mathrm{~Hz}, J_{m}=\right.$ $\left.1.5 \mathrm{~Hz}, 1 \mathrm{H}, \mathrm{H}_{\mathrm{d}}\right), 7.86\left(\mathrm{t}, J=8.0 \mathrm{~Hz}, 1 \mathrm{H}, \mathrm{H}_{\mathrm{b}}\right), 7.54\left(\mathrm{t}, J=7.6 \mathrm{~Hz}, 1 \mathrm{H}, \mathrm{H}_{\mathrm{c}}\right), 7.34\left(\mathrm{~d}, J=8.0 \mathrm{~Hz}, 1 \mathrm{H}, \mathrm{H}_{\mathrm{a}}\right), 3.96$ $\left(\mathrm{t}, J=4.8 \mathrm{~Hz}, 2 \mathrm{H}, \mathrm{H}_{\mathrm{e}}\right), 1.29\left(\mathrm{~m}, 2 \mathrm{H}, \mathrm{H}_{\mathrm{f}}\right), 0.97\left(\mathrm{q}, J=6.9 \mathrm{~Hz}, 6 \mathrm{H}, \mathrm{H}_{\mathrm{h}}\right), 0.6\left(\mathrm{~m}, 2 \mathrm{H}, \mathrm{H}_{\mathrm{g}}\right),-1.26(\mathrm{t}, J=6.9 \mathrm{~Hz}$, $\left.9 \mathrm{H}, \mathrm{H}_{\mathrm{i}}\right),-2.94\left(\mathrm{~s}, 2 \mathrm{H}, \mathrm{N} H_{\text {pyrr }}\right) .{ }^{13} \mathrm{C}$ NMR $\left(100 \mathrm{MHz}, \mathrm{CDCl}_{3}, 298 \mathrm{~K}\right) \delta: 162.8,158.5,148.5,145.4,140.0$, $136.4,134.2,131.7,130.8,121.5,119.8,114.5,103.3,65.8,52.7,51.5,22.4,5.2$. Eight quaternary carbon atoms were not detected. ${ }^{19} \mathrm{~F}$ NMR $\left(376 \mathrm{MHz}, \mathrm{CDCl}_{3}, 298 \mathrm{~K}\right) \delta:-137.8,-151.0,-161.0$.

\subsection{Synthesis of $\operatorname{Zn}(2)$.}

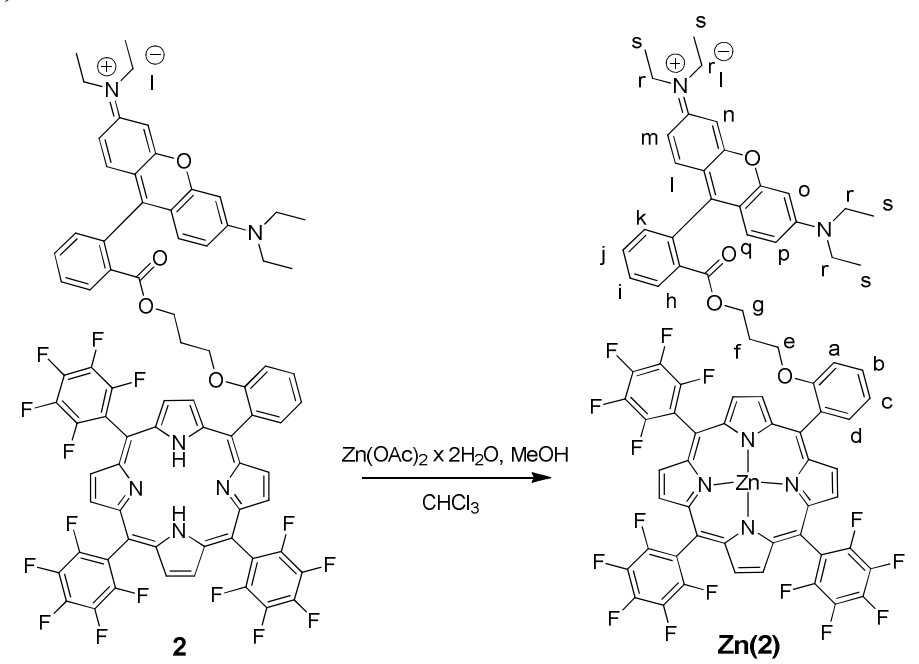

A dry $\mathrm{CH}_{3} \mathrm{OH}(13.50 \mathrm{~mL})$ solution of $\mathrm{Zn}(\mathrm{OAc})_{2} \cdot 2 \mathrm{H}_{2} \mathrm{O}(72 \mathrm{mg}, 0.33 \mathrm{mmol})$ was added to a $\mathrm{CHCl}_{3}(6.50 \mathrm{~mL})$ solution of $2(49 \mathrm{mg}, 0.033 \mathrm{mmol})$ in a $50 \mathrm{~mL}$ dried Schlenk. The mixture was refluxed under stirring for 2 hours, then the solvent was evaporated to dryness under reduced pressure and $15 \mathrm{~mL}$ of $\mathrm{CH}_{2} \mathrm{Cl}_{2}$ was added to the residue. The organic phase was washed with water $(3 \times 15 \mathrm{~mL})$, dried over $\mathrm{NaSO}_{4}$ and the filtrate was evaporated to dryness under reduced pressure yielding $\mathrm{Zn}(2)$ in a quantitative yield. Elemental Analysis: calc. for $\mathrm{C}_{75} \mathrm{H}_{48} \mathrm{~F}_{15} \mathrm{IN}_{6} \mathrm{O}_{4} \mathrm{Zn}$ : C, 62.23; H, 3.34; N, 5.81; found: C, 62.40; H, 3.71; N, 5.62. UV-Vis, $\lambda_{\max }$ $\left(\mathrm{CH}_{2} \mathrm{Cl}_{2}\right) / \mathrm{nm}\left(\log \varepsilon_{\mathrm{M}}\right): 419$ (5.05), 560 (4.99). $\lambda_{\max }(\mathrm{MeOH}) / \mathrm{nm}\left(\log \varepsilon_{\mathrm{M}}\right): 418(5.01), 561(4.83)$. LR-MS (ESI): $\mathrm{m} / \mathrm{z}\left(\mathrm{C}_{75} \mathrm{H}_{48} \mathrm{~F}_{15} \mathrm{~N}_{6} \mathrm{O}_{4} \mathrm{Zn}^{+}[\mathrm{M}]^{+}\right)$calcd. 1445.28; found 1446.67. ${ }^{1} \mathrm{H} \mathrm{NMR}\left(400 \mathrm{MHz}, \mathrm{CDCl}_{3}, 298 \mathrm{~K}\right): \delta$ $8.91\left(\mathrm{~m}, 6 \mathrm{H}, \mathrm{H}_{\text {ppyrr }}\right), 8.75\left(\mathrm{~s}, 2 \mathrm{H}, \mathrm{H}_{\text {ppyrr }}\right), 8.14\left(\mathrm{~d}, J=6.7 \mathrm{~Hz}, 1 \mathrm{H}, \mathrm{H}_{\mathrm{h}}\right), 7.76\left(\mathrm{t}, J=7.6 \mathrm{~Hz}, 1 \mathrm{H}, \mathrm{H}_{\mathrm{j}}\right), 7.44($ br s, $2 \mathrm{H}, \mathrm{H}_{\mathrm{i}}$ and $\left.\mathrm{H}_{\mathrm{a}}\right), 7.26\left(\mathrm{br} \mathrm{s}, \mathrm{H}_{\mathrm{k}}\right.$ and $\mathrm{H}_{\mathrm{c}}$ ), $7.13\left(\mathrm{br} \mathrm{s}, 1 \mathrm{H}, \mathrm{H}_{\mathrm{b}}\right), 6.88\left(\mathrm{~d}, J=7.4 \mathrm{~Hz}, 1 \mathrm{H}, \mathrm{H}_{\mathrm{d}}\right), 6.53\left(\mathrm{~s}, 2 \mathrm{H}, \mathrm{H}_{1}\right.$ and $\mathrm{H}_{\mathrm{q}}$ ), $6.41\left(\mathrm{~m}, 4 \mathrm{H}, \mathrm{H}_{\mathrm{m}}, \mathrm{H}_{\mathrm{n}}, \mathrm{H}_{\mathrm{o}}\right.$ and $\left.\mathrm{H}_{\mathrm{p}}\right), 3.48\left(\mathrm{~s}, 2 \mathrm{H}, \mathrm{H}_{\mathrm{e}}\right), 3.38$ (br s, $\left.8 \mathrm{H}, \mathrm{H}_{\mathrm{r}}\right), 1.28$ (solvent overlap $\mathrm{H}_{\mathrm{g}}$ ), $1.12(\mathrm{~s}$, $\left.12 \mathrm{H}, \mathrm{H}_{\mathrm{s}}\right), 0.62\left(\mathrm{~s}, 2 \mathrm{H}, \mathrm{H}_{\mathrm{f}}\right) .{ }^{13} \mathrm{C} \mathrm{NMR}\left(100 \mathrm{MHz}, \mathrm{CDCl}_{3}, 298 \mathrm{~K}\right): \delta 158.8,157.9,157.3,155.3,151.3,150.1$, $149.8,149.6,148.3,145.0,135.1,133.6,132.7,132.5,132.4,131.6,131.2,130.7,130.4,129.8,129.7,120.8$, 114.0, 113.0, 103.1, 102.3, 96.4, 66.1, 61.8, 46.1, 29.8, 27.2, 12.6. eight quaternary carbon atoms were not detected. ${ }^{19} \mathrm{~F}$ NMR $\left(376 \mathrm{MHz}, \mathrm{CDCl}_{3}, 298 \mathrm{~K}\right): \delta$-136.7, -137.7, 140.3, 141.3, 152.4, -153.1, -162.3 to 163.0. 


\section{3. ${ }^{1} \mathrm{H},{ }^{13} \mathrm{C}$ and ${ }^{19} \mathrm{~F}$ NMR spectra of reported compounds}

Figure 1. ${ }^{1} \mathrm{H} \mathrm{NMR}$ spectrum (400 MHz, $\mathrm{CDCl}_{3}, 298 \mathrm{~K}$ ) of porphyrin 1

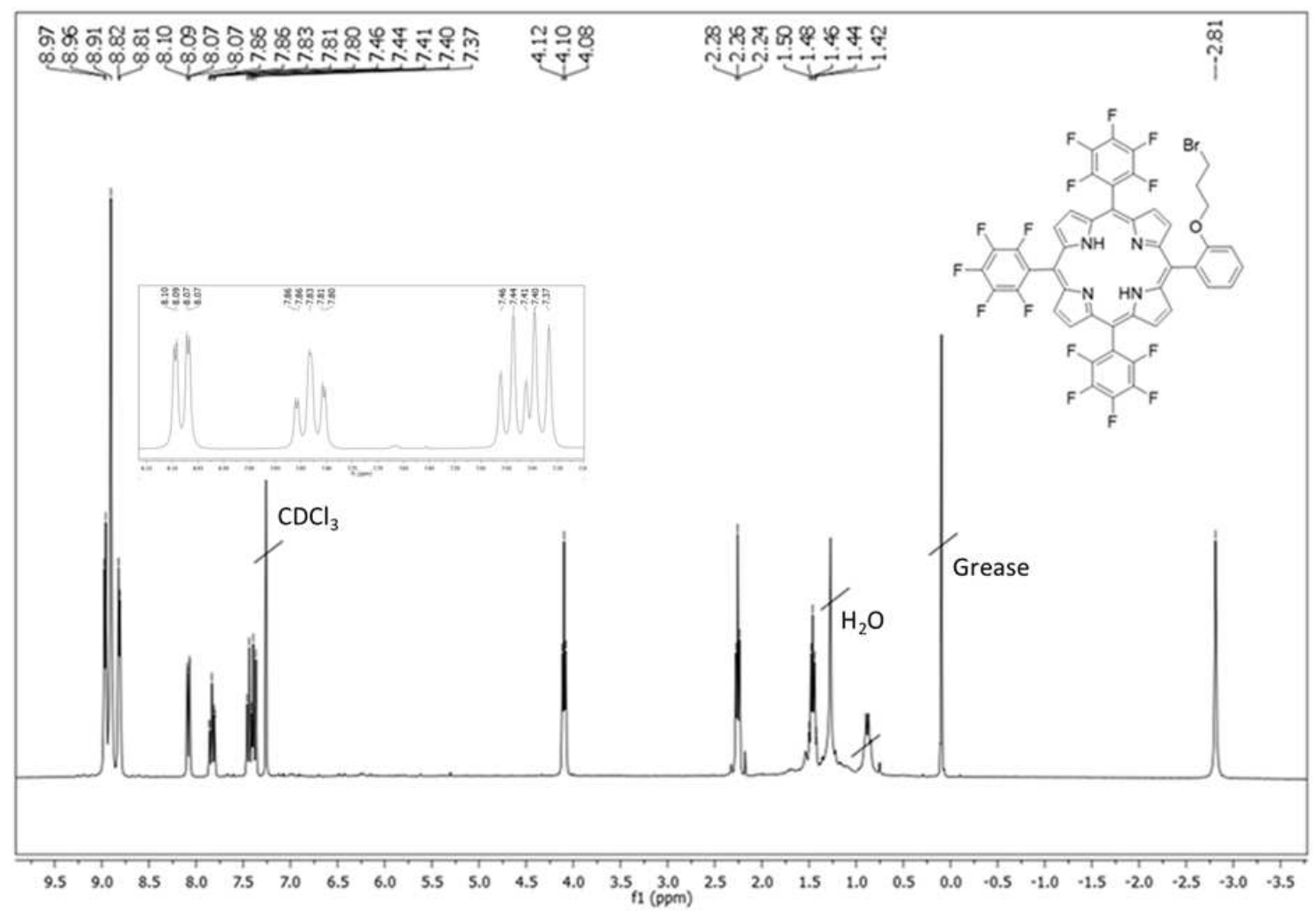

Figure $2 .{ }^{13} \mathrm{C}$ NMR spectrum (100 MHz, $\mathrm{CDCl}_{3}, 298 \mathrm{~K}$ ) of porphyrin 1

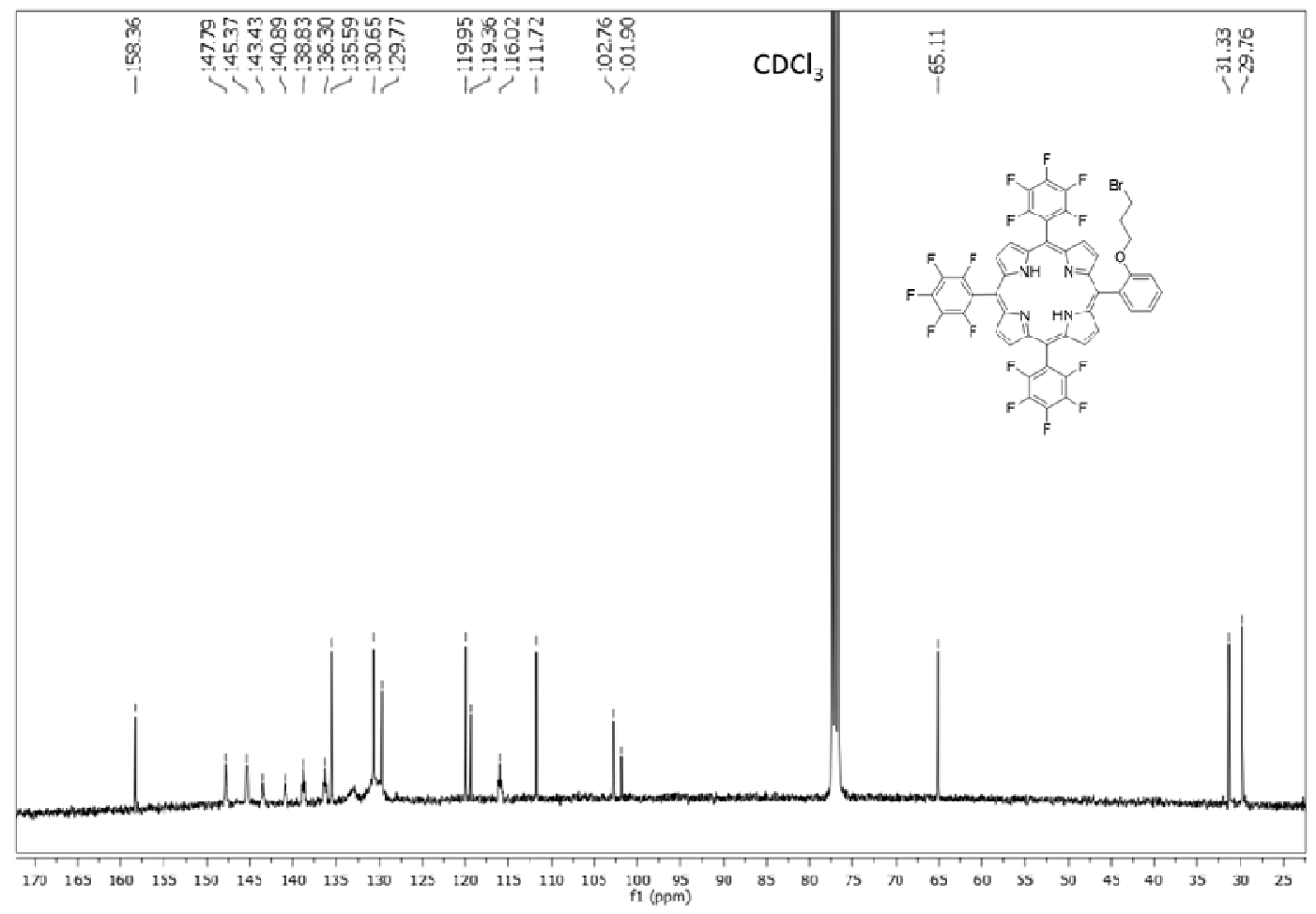


Figure $3 .{ }^{19} \mathrm{~F}$ NMR spectrum (376 MHz, $\mathrm{CDCl}_{3}, 298 \mathrm{~K}$ ) of porphyrin 1

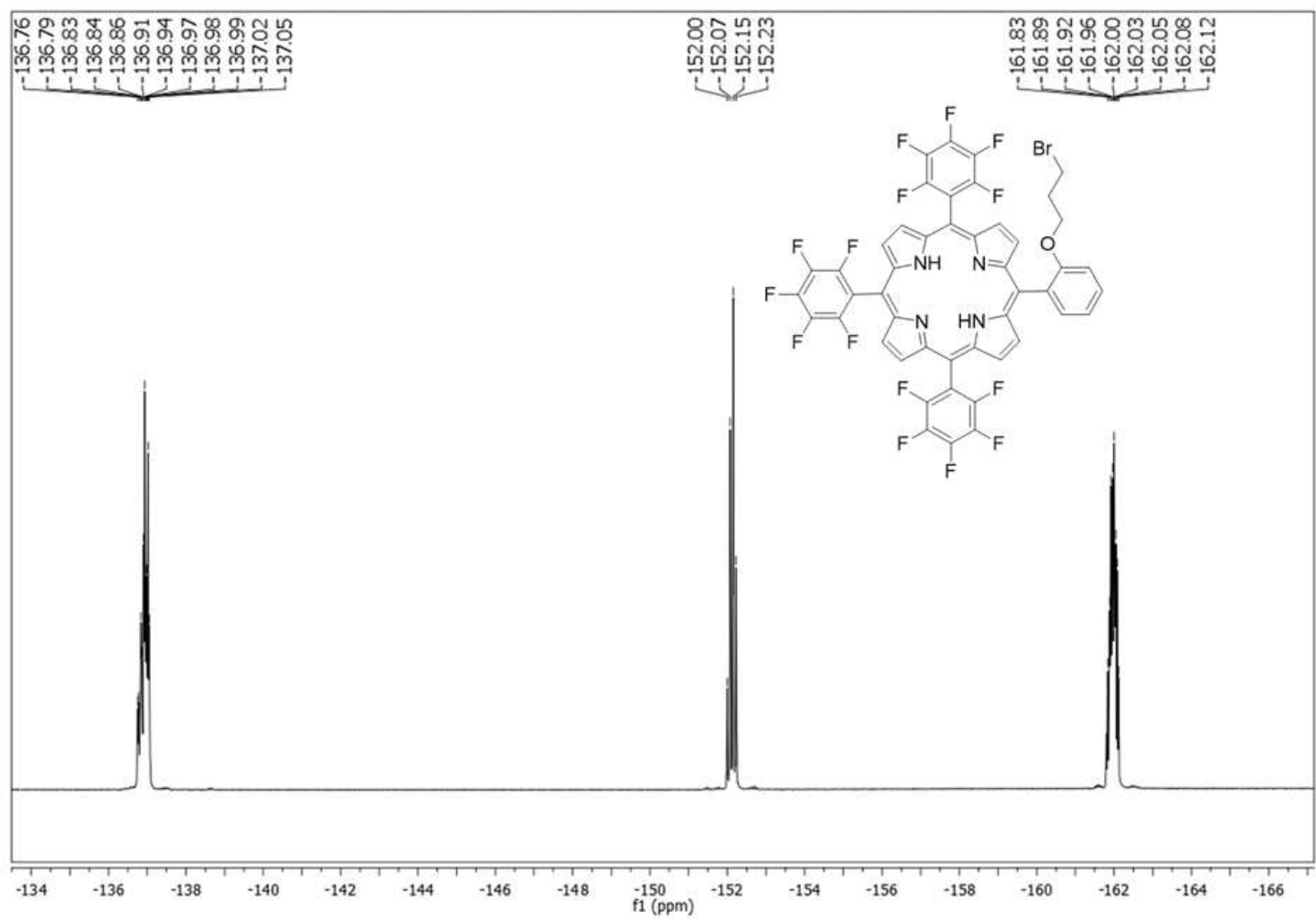

Figure 4. ${ }^{1} \mathrm{H}$ NMR spectrum (400 MHz, $\mathrm{CDCl}_{3}, 298 \mathrm{~K}$ ) of porphyrin 2

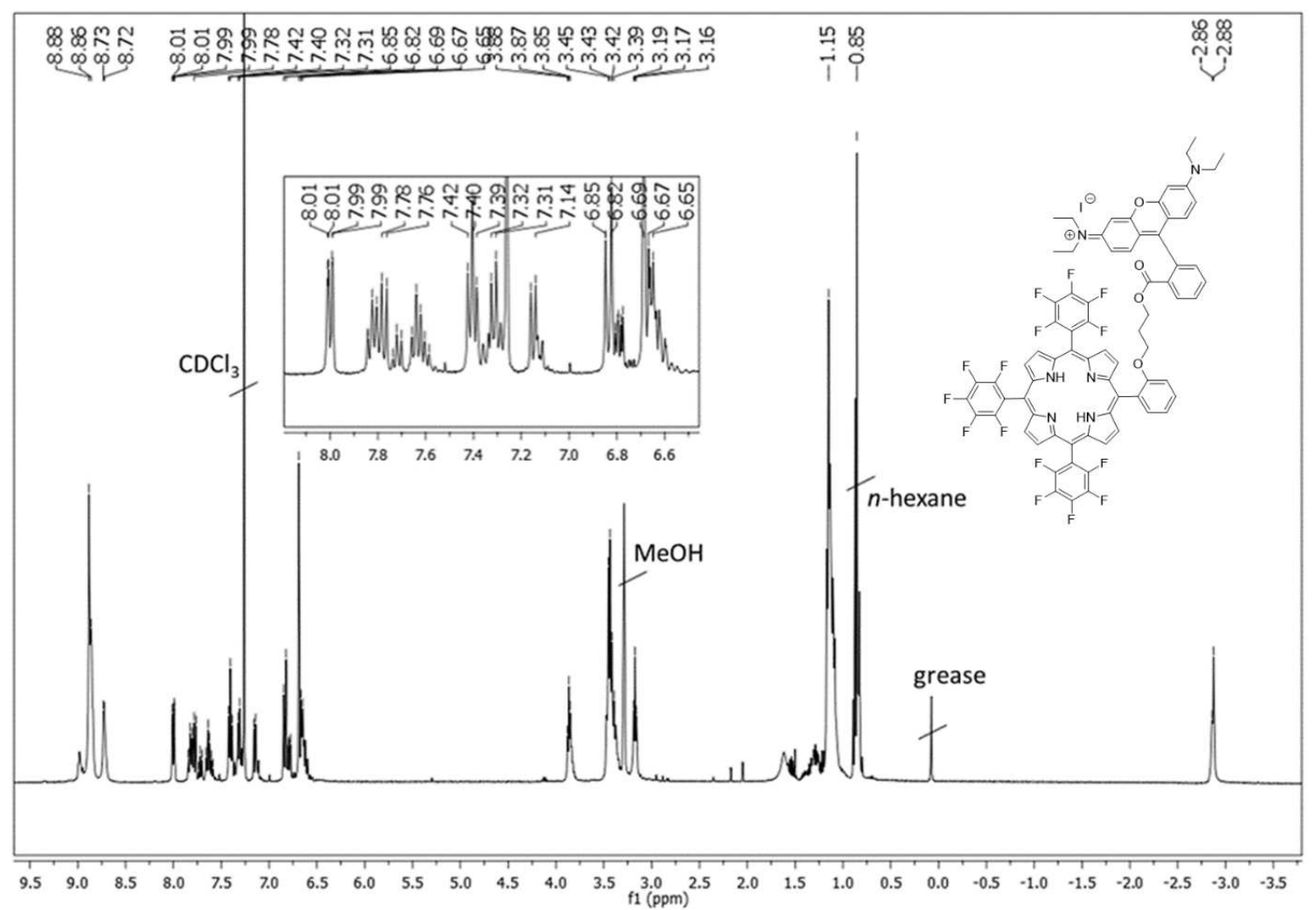


Figure $5 .{ }^{13} \mathrm{C}$ NMR spectrum (100 MHz, $\mathrm{CDCl}_{3}, 298 \mathrm{~K}$ ) of porphyrin 2

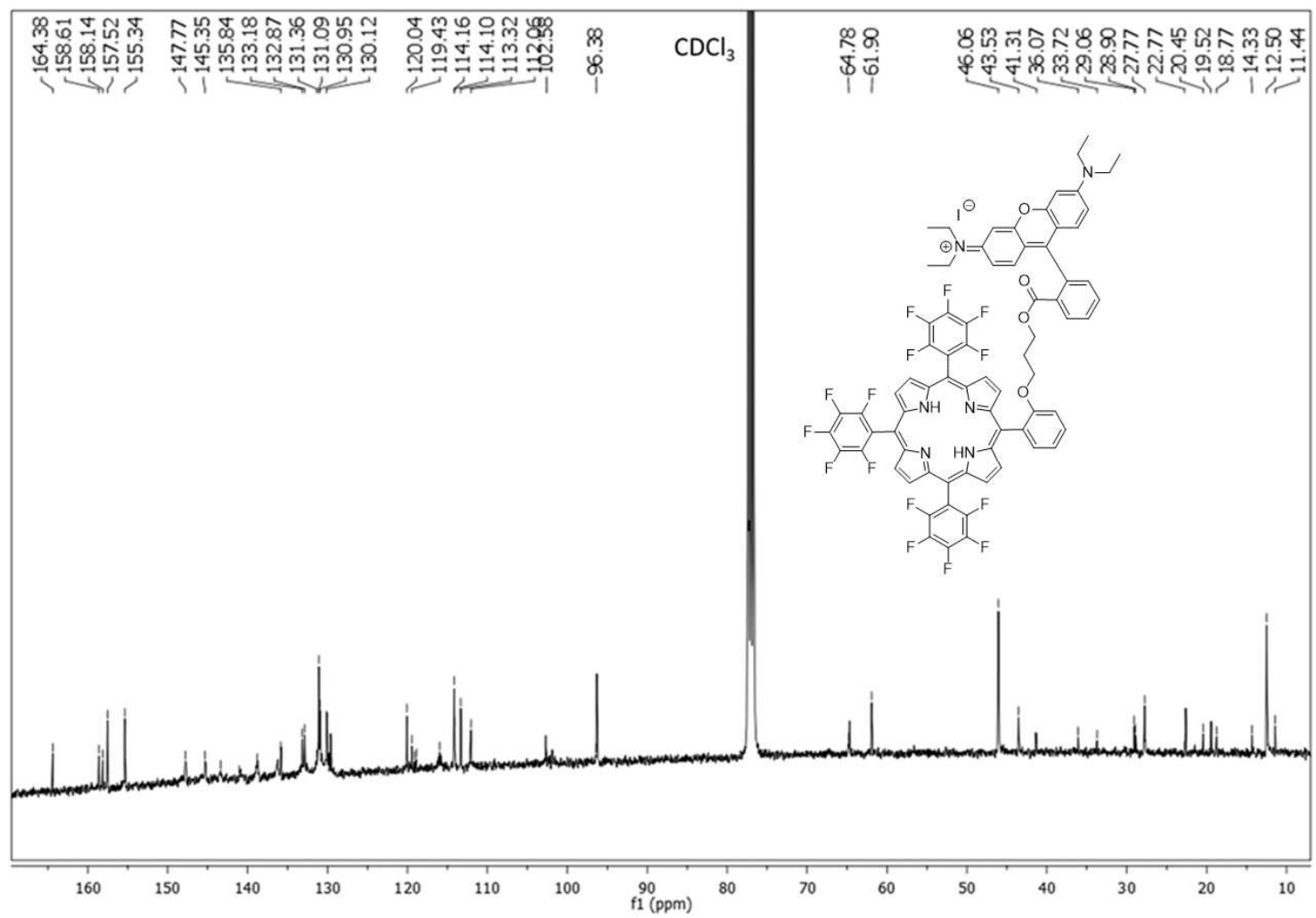

Figure 6. ${ }^{19} \mathrm{~F}$ NMR spectrum (376 MHz, $\mathrm{CDCl}_{3}, 298 \mathrm{~K}$ ) of porphyrin 2

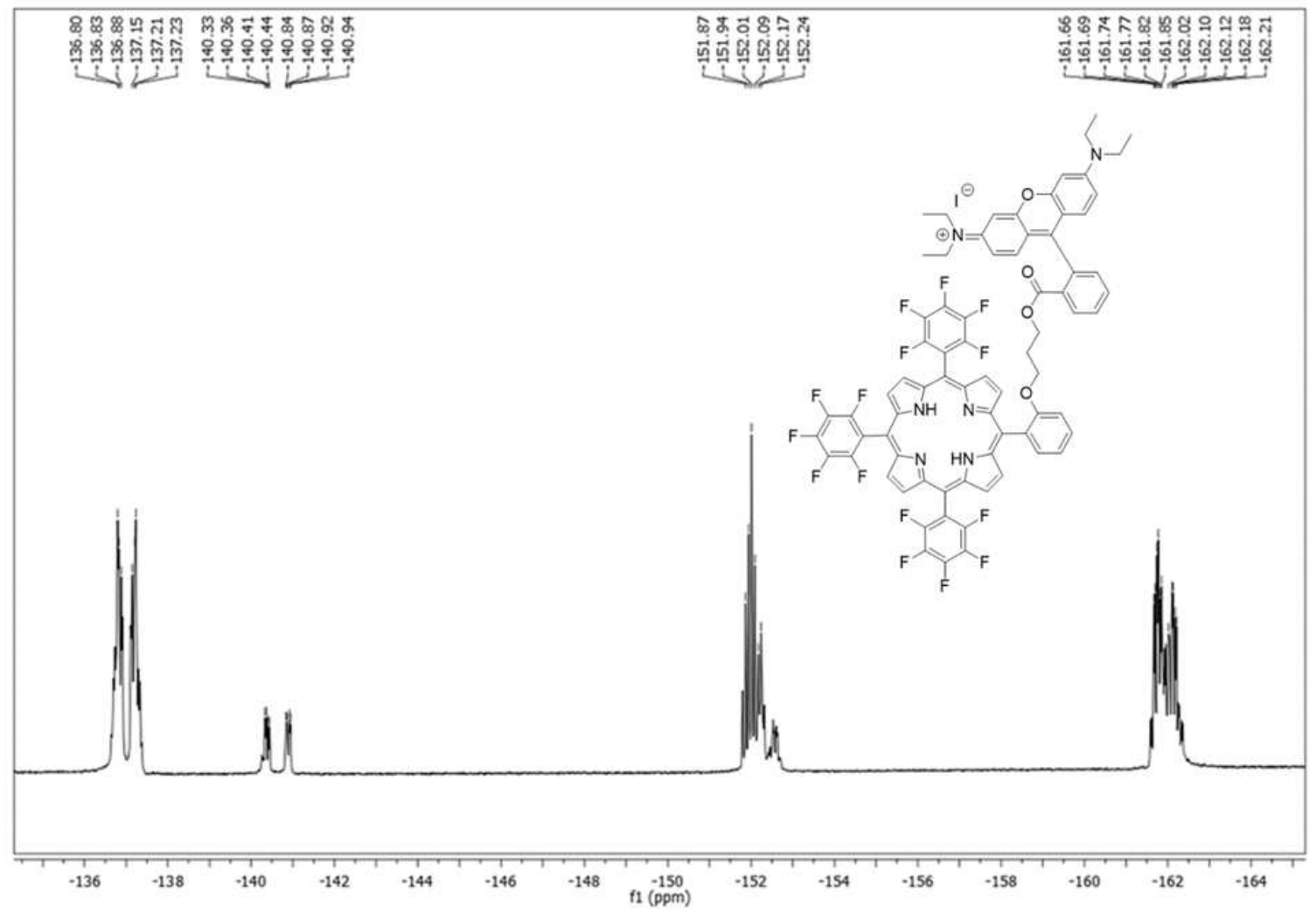


Figure 7. ${ }^{1} \mathrm{H}$ NMR spectrum ( $400 \mathrm{MHz}, \mathrm{CDCl}_{3}, 298 \mathrm{~K}$ ) of porphyrin 3

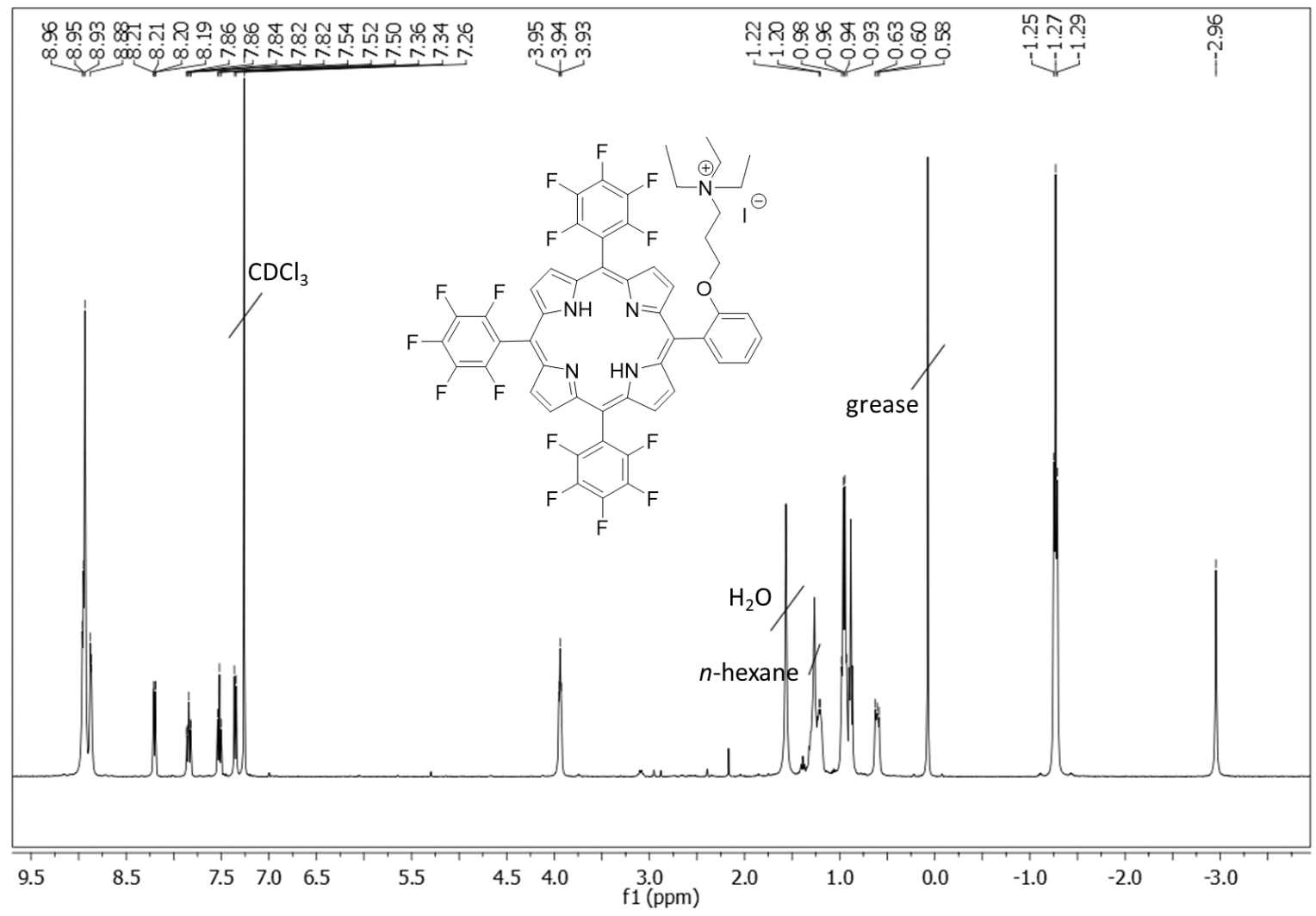

Figure $8 .{ }^{13} \mathrm{C}$ NMR spectrum (100 MHz, $\left.\mathrm{CDCl}_{3}, 298 \mathrm{~K}\right)$ of porphyrin 3

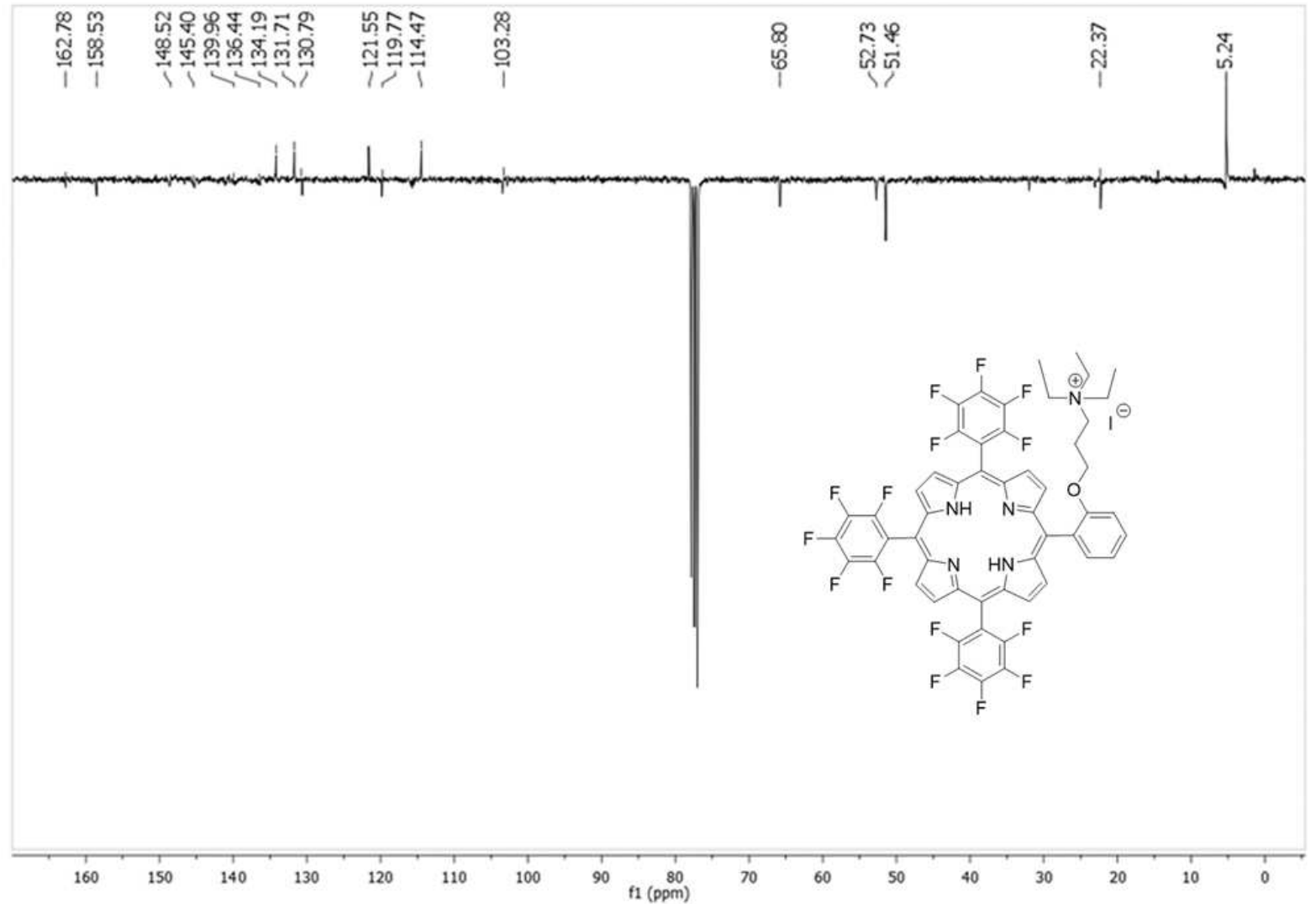


Figure 9. ${ }^{19} \mathrm{~F}$ NMR spectrum (376 MHz, $\left.\mathrm{CDCl}_{3}, 298 \mathrm{~K}\right)$ of porphyrin 3

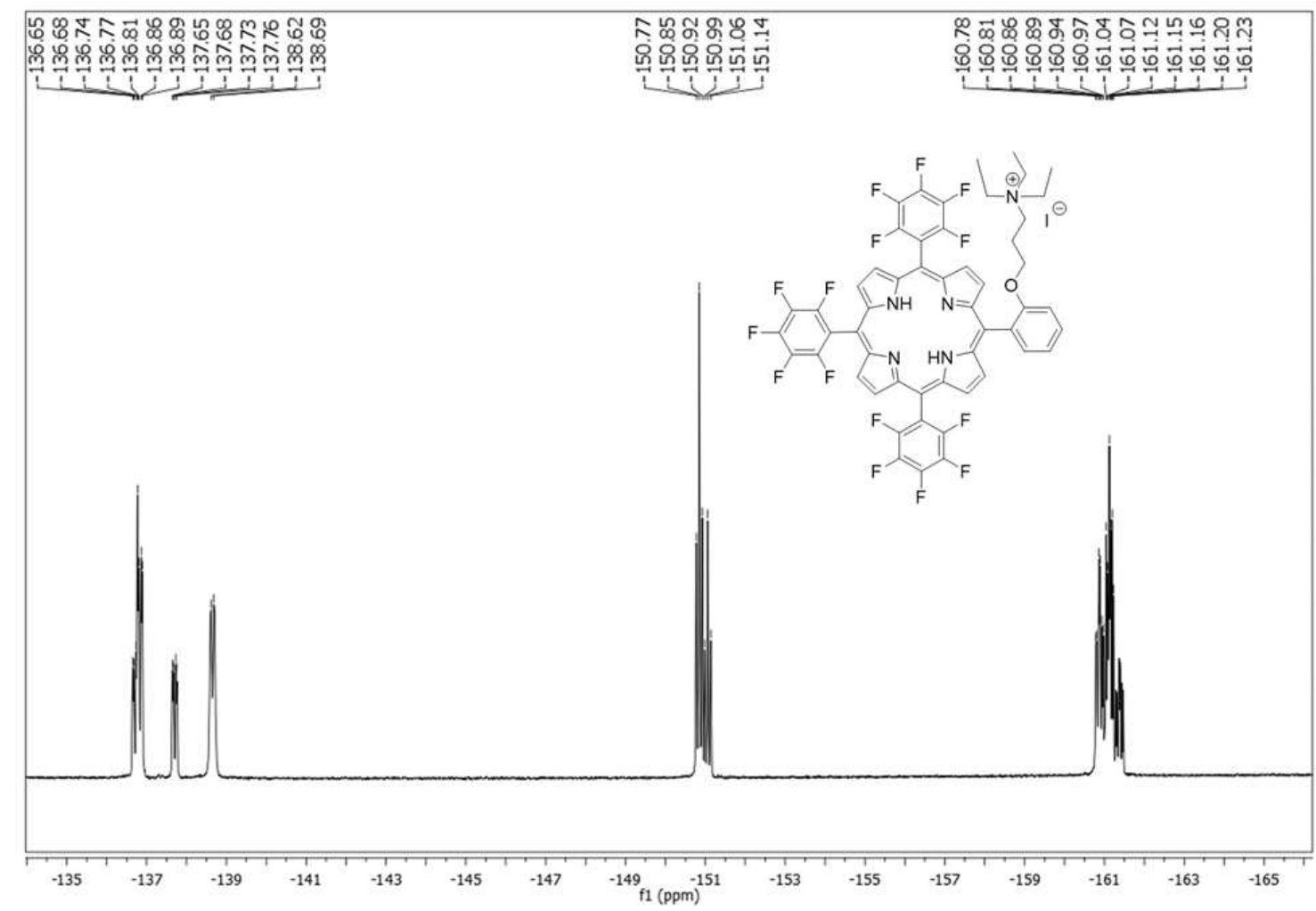

Figure 10. ${ }^{1} \mathrm{H}$ NMR spectrum (400 MHz, $\mathrm{CDCl}_{3}, 298 \mathrm{~K}$ ) of porphyrin $\mathrm{Zn}(2)$

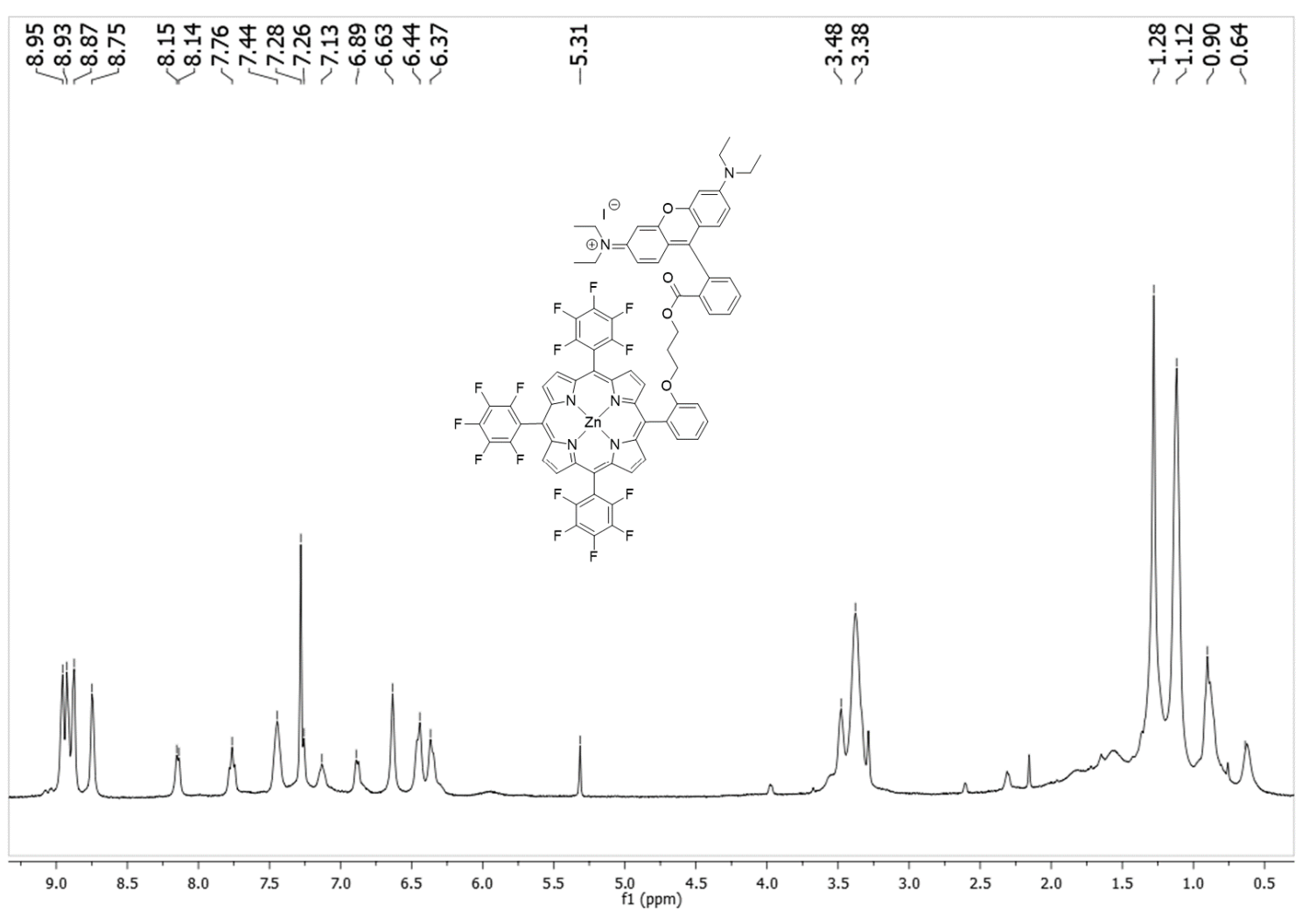


Figure $11 .{ }^{13} \mathrm{C}$ NMR spectrum (100 MHz, $\left.\mathrm{CDCl}_{3}, 298 \mathrm{~K}\right)$ of porphyrin $\mathrm{Zn}(2)$

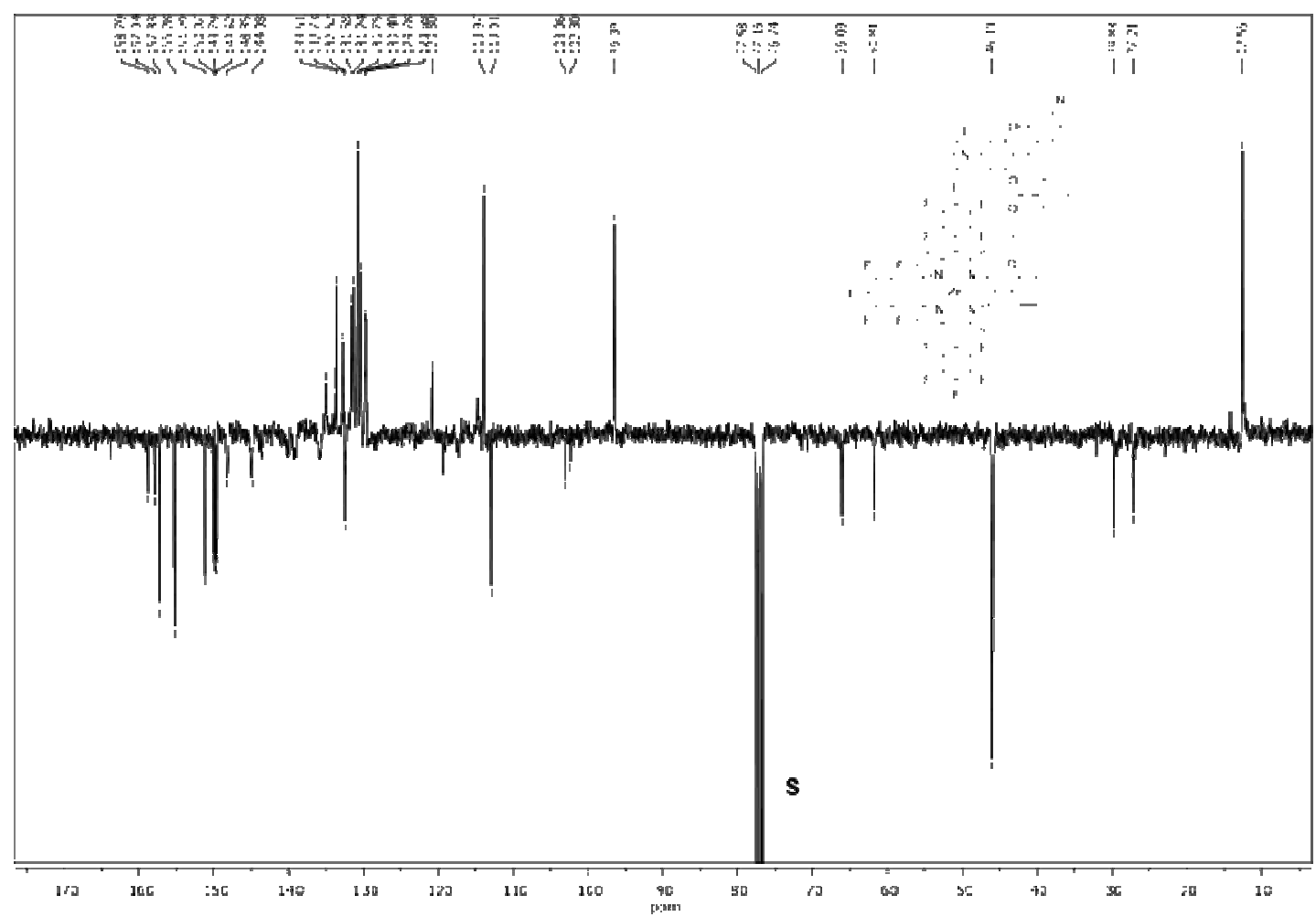

Figure 12. ${ }^{19} \mathrm{~F}$ NMR spectrum (376 MHz, $\mathrm{CDCl}_{3}, 298 \mathrm{~K}$ ) of porphyrin $\mathrm{Zn}(2)$

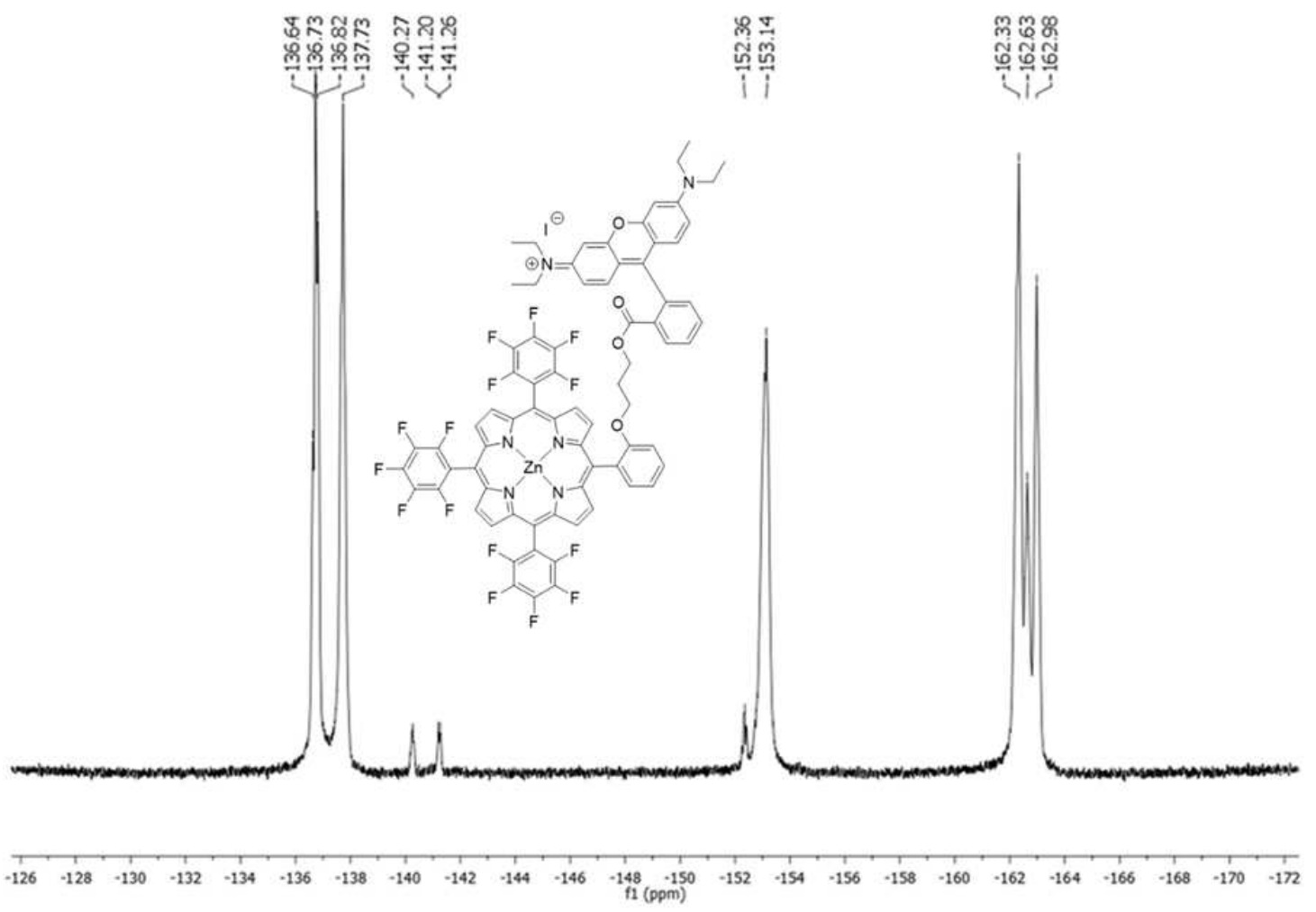




\section{UV-Vis spectra}

Figure 13. UV-vis spectra of $\mathbf{1}, \mathbf{2}, \mathbf{3}, \mathrm{Zn}(\mathbf{2})$ and Rhodamine B in $\mathrm{MeOH}$.
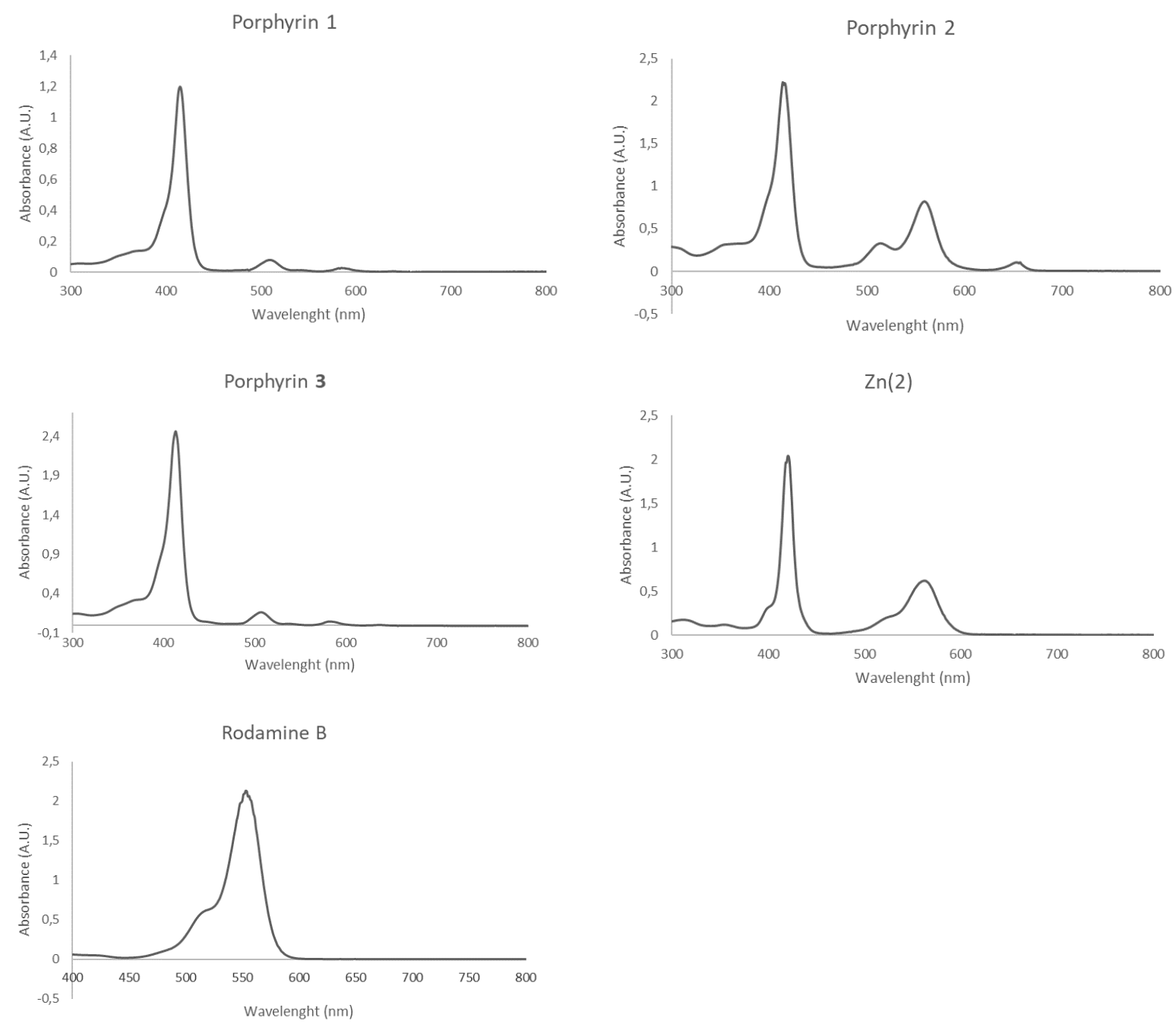


\section{UV-Vis binding tests}

Binding constant measurements were carried out on a Cary 100 Spectrophotometer at $298.0( \pm 0.5)^{\circ} \mathrm{C}$ by adding consecutive aliquots of a (DCF)Na solution (1.00 mM) to $2.0 \mathrm{~mL}$ of $1: 1 \mathrm{MeOH} / \mathrm{H}_{2} \mathrm{O}$ (v:v) receptor (2 or $\mathrm{Zn}(2))$ solution $(10.0 \mu \mathrm{M})$. The corresponding spectra was acquired after every (DCF)Na addition. The (DCF)Na solution was prepared by dissolving the required amount of salt into $10.0 \mathrm{~mL}$ of $10.0 \mu \mathrm{M}$ porphyrin solution, in order to keep the concentration of the receptor to a constant value throughout the titration. The absorbance values at $419 \mathrm{~nm}$ were plotted against (DCF) Na concentration and acquired data fitted to Equation $1^{8}$ for a 1:1 molecular complex formation by using a non-linear regression fit program (KaleidaGraph ${ }^{\circledR} 4.1$ Synergy Software). Experiments were performed in duplicate and obtained results showed a very good reproducibility. The same procedure was followed in the presence of sodium phenylacetate, sodium salicylate, L tyrosine sodium salt, sodium sarcosinate, L-alanine sodium salt as well as for Fluorescence and Resonance Light Scattering (RLS) studies.

\section{Equation 1}

$\frac{A_{0}-A}{A-A_{\infty}}=\frac{[S\rfloor_{t}+[L\rfloor_{a}+1 / K_{\text {hinding }}-\sqrt{\left([S]_{t}+[L\rfloor_{a}+1 / K_{\text {hinding }}\right)^{2}-4[S]_{t}[L\rfloor_{a}}}{2[S]_{t}}$

Figure 14. UV-vis binding test with porphyrin 2

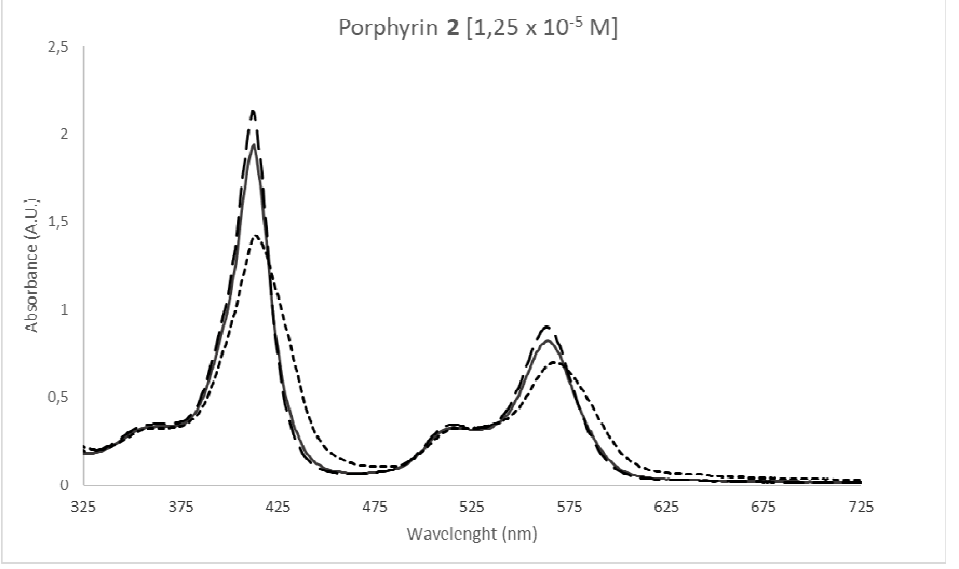

Figure 15. UV-vis binding test with porphyrin $\mathrm{Zn}(\mathbf{2})$

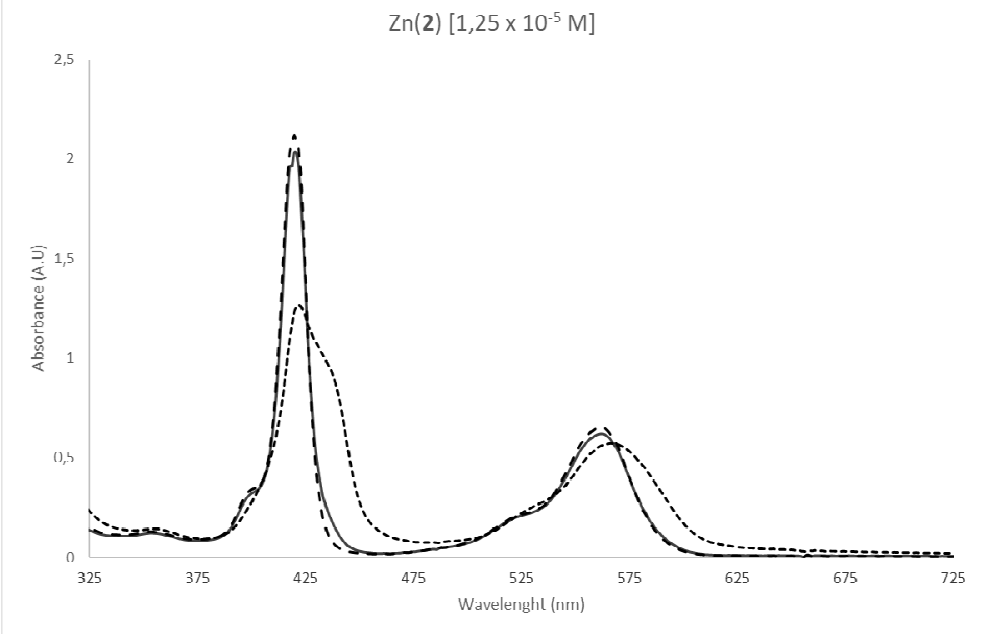


Figure 16. UV-Vis spectral pattern variation of $2\left(10 \mu \mathrm{M} ; \mathrm{MeOH} / \mathrm{H}_{2} \mathrm{O}\right.$ 1:1 v:v) upon addition of (DCF)Na and equimolar amount of sodium salicylate $(\mathrm{SalONa})$.

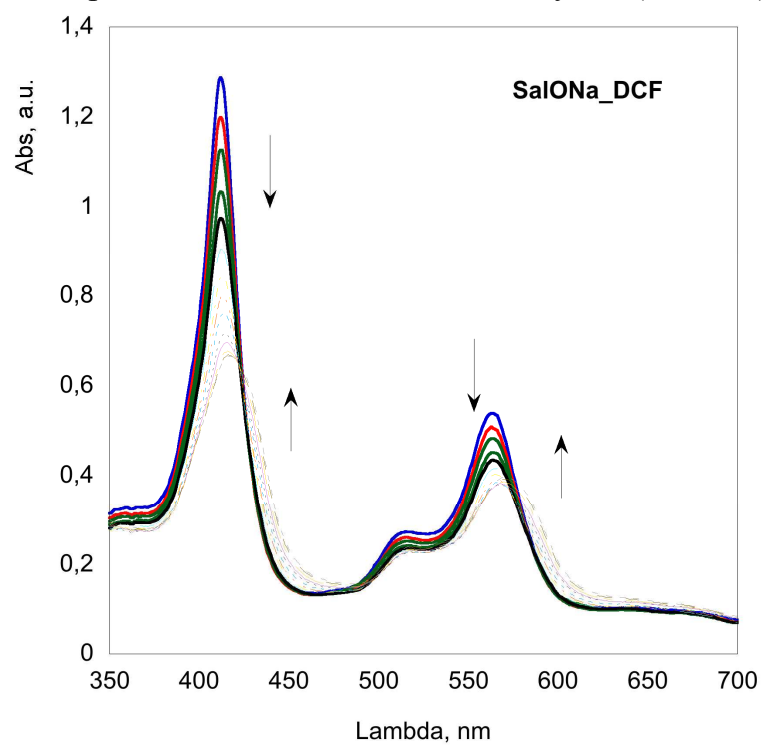

Figure 17. Non-linear regression fit, and calculated binding constant value (Langmuir type equation, see above) for the 2@DCF formation in the presence of equimolar amount of sodium salicylate.

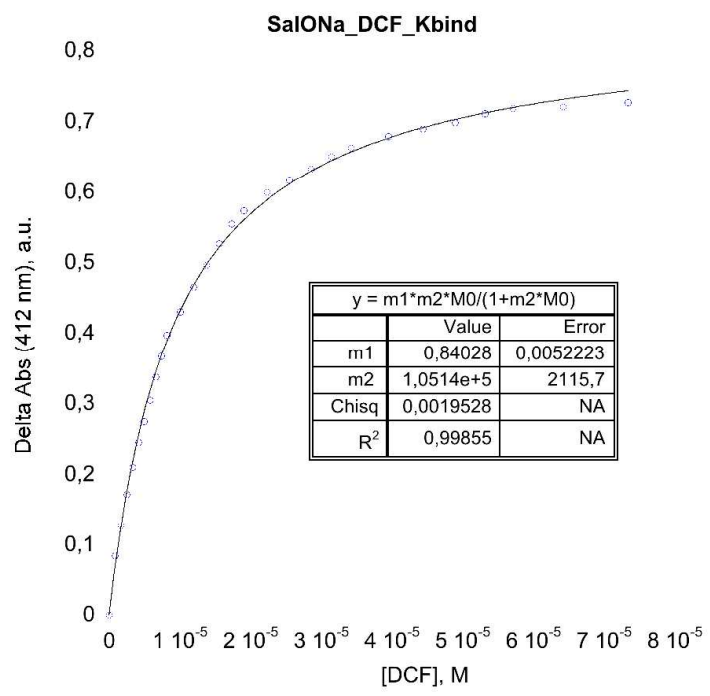




\section{Fluorescence experiments}

Figure 18. Fluorescence emission spectrum of 2 at $\lambda_{\mathrm{exc}}=412 \mathrm{~nm}$.

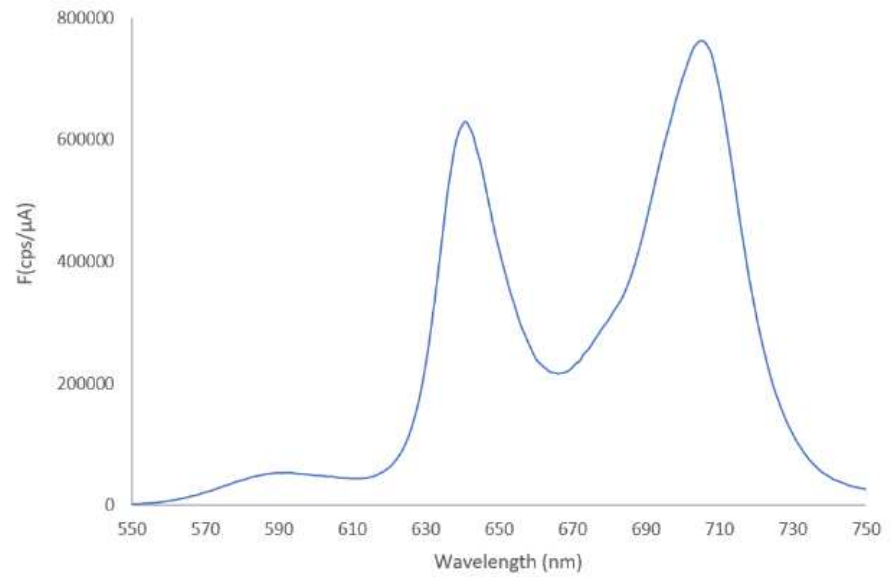

Figure 19. Fluorescence excitation spectrum of 2 at $\lambda_{\mathrm{em}}=590 \mathrm{~nm}$ (Rhodamine emission)

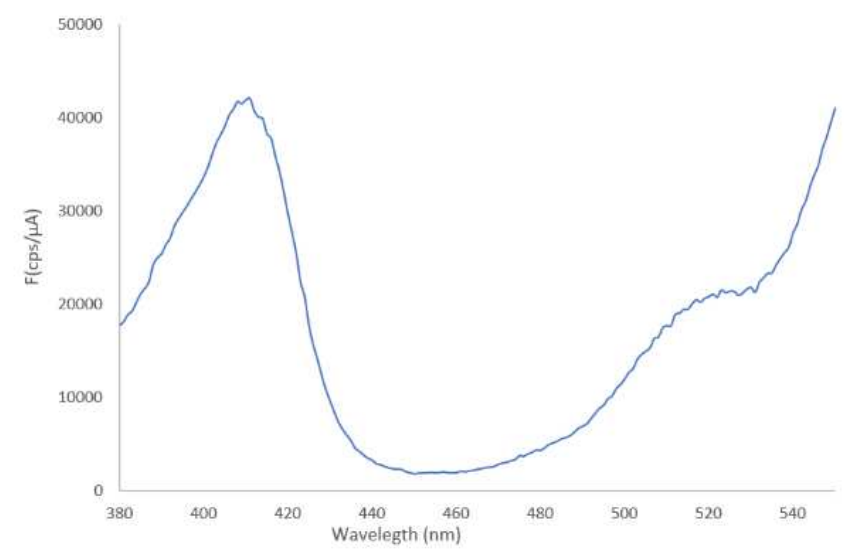

Figure 20. Fluorescence excitation spectrum of 2 at $\lambda_{\mathrm{em}}=705 \mathrm{~nm}$ (Porphyrin emission)

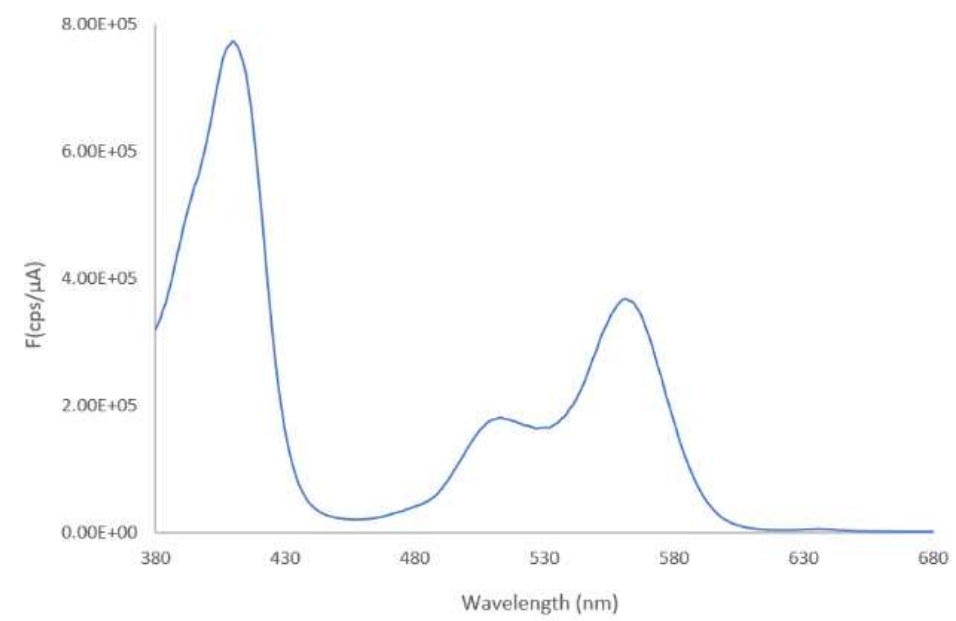


Figure 21. Fluorescence emission spectra of 2 for increasing concentrations of DCF. A: $\lambda_{\mathrm{ex}}=412 \mathrm{~nm}$ (Porphyrin absorption); B: $\lambda_{\mathrm{ex}}=555 \mathrm{~nm}$ (Rhodamine preferential absorption).
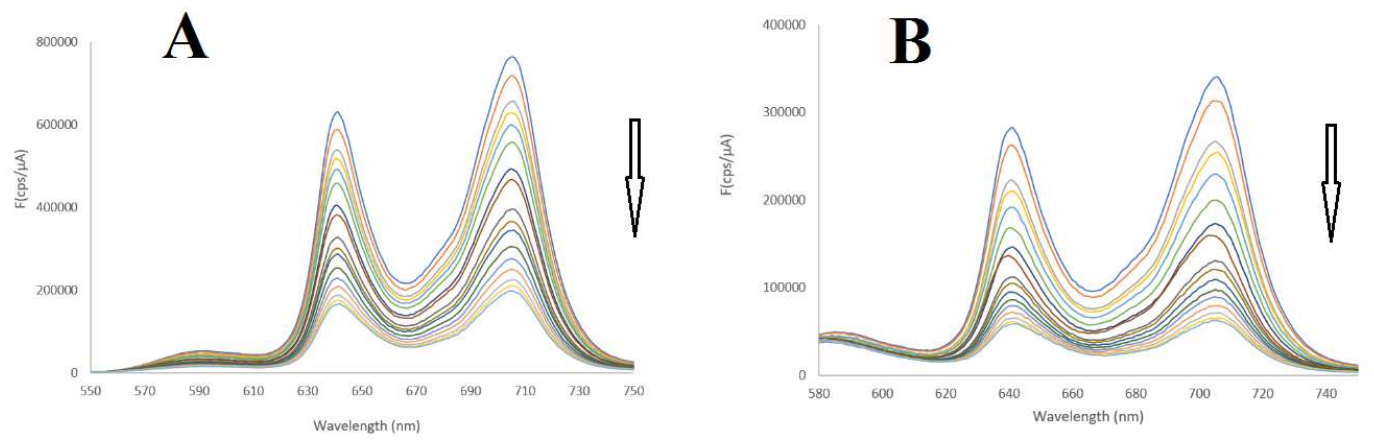

Figure 22. 2/DCF binding isotherm from fluorescence intensities of $\mathbf{2}$ for different (DCF)Na concentrations. The analogous of Equation 1 was applied.

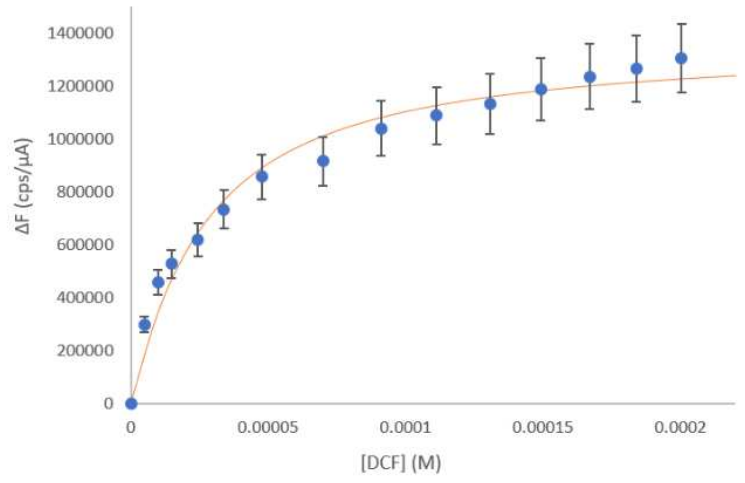

Figure 23. Fluorescence time decays of 2 for different $2 / \mathrm{DCF}$ concentration ratios $\left(\lambda_{\mathrm{ex}}=342 \mathrm{~nm} ; \lambda_{\mathrm{em}}=645\right.$ $\mathrm{nm})$.

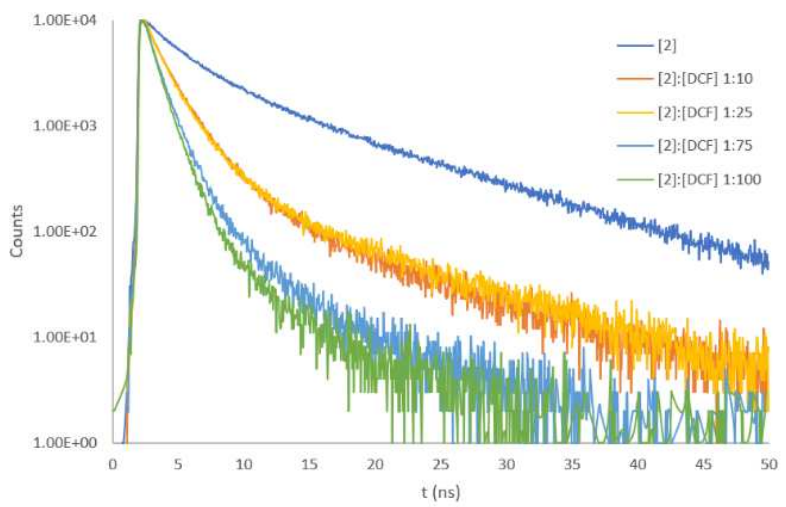

Table 1. Time decay parameters of $\mathbf{2}$ (porphyrin emission) for different $\mathbf{2} / \mathrm{DCF}$ concentration ratios $\left(\lambda_{\mathrm{ex}}=342\right.$ $\left.\mathrm{nm} ; \lambda_{\mathrm{em}}=645 \mathrm{~nm}\right)$.

\begin{tabular}{lcccccccc}
\hline \multicolumn{1}{c}{$[2]:[\mathrm{DCF}]$} & $\tau_{1}(\mathrm{~ns})$ & $\alpha_{1}$ & $\tau_{2}(\mathrm{~ns})$ & $\alpha_{2}$ & $\tau_{3}(\mathrm{~ns})$ & $\alpha_{3}$ & $\langle\tau\rangle(\mathrm{ns})$ & $\chi^{2}$ \\
\hline $1: 0$ & 11.6 & 0.38 & 3.45 & 0.46 & 1.0 & 0.17 & 6.3 & 1.303 \\
$1: 10$ & 10.2 & 0.02 & 2.2 & 0.52 & 0.95 & 0.46 & 1.8 & 1.154 \\
$1: 25$ & 10.0 & 0.03 & 2.1 & 0.48 & 0.88 & 0.49 & 1.7 & 1.031 \\
$1: 75$ & 6.1 & 0.01 & 1.5 & 0.41 & 0.72 & 0.58 & 1.1 & 1.052 \\
$1: 100$ & 5.0 & 0.01 & 1.2 & 0.50 & 0.64 & 0.49 & 1.0 & 0.913 \\
\hline
\end{tabular}




\section{X-ray single-crystal structure determination.}

Crystal data for $\mathbf{3}$ are reported in Table 2. All hydrogen atoms of porphyrin molecule, except the $\mathrm{H}$ atoms of methyl groups, were located from the difference Fourier map and refined freely with isotropic displacement parameters. Methyl hydrogens and $\mathrm{H}$-atoms of the guest molecule ( $n$-hexane) were placed in geometrically calculated positions and included in the refinement using a riding model in conjunction with a $U_{\text {iso }}(\mathrm{H})=1.2$ $U_{\text {eq }}\left(\mathrm{CH}_{2}, \mathrm{CH}\right)$ or $1.5 U_{\text {eq }}\left(\mathrm{CH}_{3}\right)$ constraint. All non-hydrogen atoms were refined with full occupancy and anisotropic displacement parameters except for hexane carbon atoms. ISOR instruction was used for F2 atom as otherwise it went non-positive definite. The solvate hexane molecule was find to be disordered and refined isotropically over two positions using a suitable model $(49.7,50.1 \%)$. Geometry constraints (SADI) were used to keep reasonable bond distances.

Table 2. Crystal data and structure refinement for $\mathbf{3}$.

$\begin{array}{ll}\text { Chemical formula } & {\left[\mathrm{C}_{53} \mathrm{H}_{35} \mathrm{~F}_{15} \mathrm{~N}_{5} \mathrm{O}\right] \mathrm{I} \cdot 0.5\left(\mathrm{C}_{6} \mathrm{H}_{14}\right)} \\ \text { Empirical formula } & \mathrm{C}_{56} \mathrm{H}_{42} \mathrm{~F}_{15} \mathrm{I} \mathrm{N}_{5} \mathrm{O} \\ \text { Formula weight }\left(\mathrm{g} \cdot \mathrm{mol}^{-1}\right) & 1212.85 \\ \text { Temperature }(\mathrm{K}) & 150(2) \\ \text { Wavelength }(\AA) & 0.71073 \\ \text { Crystal system } & \text { Triclinic } \\ \text { Space group } & P-1 \\ \mathrm{a} / \AA & 11.465(3) \\ \mathrm{b} / \AA & 15.353(4) \\ \mathrm{c} / \AA & 16.170(5) \\ \alpha(\mathrm{deg}) & 103.382(3) \\ \beta(\text { deg}) & 90.662(3) \\ \gamma(\text { deg}) & 106.684(3) \\ \mathrm{V} / \AA^{3} & 14687(2) \\ Z & 12 \\ D_{\text {calc. }}\left(\mathrm{g} \cdot \mathrm{cm}{ }^{-3}\right) & 1.524 \\ \mu\left(\mathrm{mm}{ }^{-1}\right) & 0.704 \\ \theta \text { range for data collection }(\mathrm{deg}) & 1.3 \text { to } 25.0 \\ \text { Index ranges } & -13<=\mathrm{h}<=13,-18<=\mathrm{k}<=18,-19<=1<=19 \\ \text { Reflections collected } & 23475 \\ \text { Independent reflections } & 9334[\mathrm{R}(\mathrm{int})=0.0383] \\ \text { Completeness to theta } & 99.9 \% \\ \text { Data/restraints/parameters } & 9334 / 21 / 775 \\ F(000) & 1218 \\ \text { Goodness-of-fit on } \mathrm{F}^{2} & 1.039 \\ \text { Final } R \text { indices }[\mathrm{I}>2 \text { sigma(I) } & R_{1}=0.0580, \mathrm{w} R_{2}=0.1461 \\ R \text { indices (all data) } & R_{1}=0.0855, \mathrm{w} R_{2}=0.1719 \\ \Delta \rho \text { max,min }\left(\mathrm{e} \cdot \AA^{-3}\right) & 2.432 \text { and }-0.775 \\ & \end{array}$


Table 3. Selected bond lengths $[\AA]$ and angles $\left[^{\circ}\right]$ for 3.

\begin{tabular}{|c|c|c|c|}
\hline $\mathrm{N}(1)-\mathrm{C}(1)$ & $1.365(6)$ & $\mathrm{N}(1)-\mathrm{C}(4)-\mathrm{C}(5)$ & $125.7(5)$ \\
\hline $\mathrm{N}(1)-\mathrm{C}(4)$ & $1.365(7)$ & $\mathrm{N}(1)-\mathrm{C}(4)-\mathrm{C}(3)$ & $109.6(5)$ \\
\hline $\mathrm{N}(2)-\mathrm{C}(6)$ & $1.362(7)$ & $\mathrm{C}(5)-\mathrm{C}(4)-\mathrm{C}(3)$ & $124.6(5)$ \\
\hline $\mathrm{N}(2)-\mathrm{C}(9)$ & $1.363(7)$ & $\mathrm{C}(4)-\mathrm{C}(5)-\mathrm{C}(6)$ & $126.8(5)$ \\
\hline $\mathrm{N}(3)-\mathrm{C}(14)$ & $1.364(6)$ & $C(4)-C(5)-C(21)$ & $117.4(5)$ \\
\hline $\mathrm{N}(3)-\mathrm{C}(11)$ & $1.370(6)$ & $C(6)-C(5)-C(21)$ & $115.6(5)$ \\
\hline $\mathrm{N}(4)-\mathrm{C}(16)$ & $1.364(7)$ & $\mathrm{N}(2)-\mathrm{C}(6)-\mathrm{C}(5)$ & $125.5(5)$ \\
\hline $\mathrm{N}(4)-\mathrm{C}(19)$ & $1.372(6)$ & $\mathrm{N}(2)-\mathrm{C}(6)-\mathrm{C}(7)$ & $107.5(5)$ \\
\hline$C(1)-C(20)$ & $1.401(7)$ & $\mathrm{C}(5)-\mathrm{C}(6)-\mathrm{C}(7)$ & $126.9(5)$ \\
\hline $\mathrm{C}(1)-\mathrm{C}(2)$ & $1.443(8)$ & $\mathrm{C}(8)-\mathrm{C}(7)-\mathrm{C}(6)$ & $108.0(5)$ \\
\hline $\mathrm{C}(2)-\mathrm{C}(3)$ & $1.330(8)$ & $\mathrm{C}(7)-\mathrm{C}(8)-\mathrm{C}(9)$ & $107.8(5)$ \\
\hline $\mathrm{C}(3)-\mathrm{C}(4)$ & $1.445(8)$ & $\mathrm{N}(2)-\mathrm{C}(9)-\mathrm{C}(10)$ & $126.6(4)$ \\
\hline$C(4)-C(5)$ & $1.383(8)$ & $\mathrm{N}(2)-\mathrm{C}(9)-\mathrm{C}(8)$ & $107.2(5)$ \\
\hline$C(5)-C(6)$ & $1.396(8)$ & $\mathrm{C}(10)-\mathrm{C}(9)-\mathrm{C}(8)$ & $126.2(5)$ \\
\hline$C(5)-C(21)$ & $1.504(7)$ & $\mathrm{C}(9)-\mathrm{C}(10)-\mathrm{C}(11)$ & $124.7(5)$ \\
\hline$C(6)-C(7)$ & $1.421(8)$ & $\mathrm{C}(9)-\mathrm{C}(10)-\mathrm{C}(27)$ & $117.5(4)$ \\
\hline $\mathrm{C}(7)-\mathrm{C}(8)$ & $1.349(8)$ & $\mathrm{C}(11)-\mathrm{C}(10)-\mathrm{C}(27)$ & $117.5(5)$ \\
\hline $\mathrm{C}(8)-\mathrm{C}(9)$ & $1.428(7)$ & $\mathrm{N}(3)-\mathrm{C}(11)-\mathrm{C}(10)$ & $126.0(5)$ \\
\hline$C(9)-C(10)$ & $1.396(7)$ & $\mathrm{N}(3)-\mathrm{C}(11)-\mathrm{C}(12)$ & $109.8(4)$ \\
\hline$C(10)-C(11)$ & $1.398(7)$ & $\mathrm{C}(10)-\mathrm{C}(11)-\mathrm{C}(12)$ & $124.1(5)$ \\
\hline$C(10)-C(27)$ & $1.489(7)$ & $\mathrm{C}(13)-\mathrm{C}(12)-\mathrm{C}(11)$ & $107.3(5)$ \\
\hline $\mathrm{C}(11)-\mathrm{C}(12)$ & $1.442(8)$ & $\mathrm{C}(12)-\mathrm{C}(13)-\mathrm{C}(14)$ & $106.7(5)$ \\
\hline $\mathrm{C}(12)-\mathrm{C}(13)$ & $1.340(8)$ & $\mathrm{N}(3)-\mathrm{C}(14)-\mathrm{C}(15)$ & $125.2(5)$ \\
\hline $\mathrm{C}(13)-\mathrm{C}(14)$ & $1.446(7)$ & $\mathrm{N}(3)-\mathrm{C}(14)-\mathrm{C}(13)$ & $110.1(4)$ \\
\hline$C(14)-C(15)$ & $1.398(7)$ & $\mathrm{C}(15)-\mathrm{C}(14)-\mathrm{C}(13)$ & $124.6(5)$ \\
\hline $\mathrm{C}(15)-\mathrm{C}(16)$ & $1.392(7)$ & $\mathrm{C}(16)-\mathrm{C}(15)-\mathrm{C}(14)$ & $126.9(5)$ \\
\hline $\mathrm{C}(15)-\mathrm{C}(33)$ & $1.499(7)$ & $\mathrm{C}(16)-\mathrm{C}(15)-\mathrm{C}(33)$ & $116.5(4)$ \\
\hline $\mathrm{C}(16)-\mathrm{C}(17)$ & $1.430(7)$ & $\mathrm{C}(14)-\mathrm{C}(15)-\mathrm{C}(33)$ & $116.6(5)$ \\
\hline $\mathrm{C}(17)-\mathrm{C}(18)$ & $1.347(8)$ & $\mathrm{N}(4)-\mathrm{C}(16)-\mathrm{C}(15)$ & $125.3(5)$ \\
\hline $\mathrm{C}(18)-\mathrm{C}(19)$ & $1.433(7)$ & $\mathrm{N}(4)-\mathrm{C}(16)-\mathrm{C}(17)$ & $108.0(4)$ \\
\hline $\mathrm{C}(19)-\mathrm{C}(20)$ & $1.391(7)$ & $\mathrm{C}(15)-\mathrm{C}(16)-\mathrm{C}(17)$ & $126.7(5)$ \\
\hline $\mathrm{C}(20)-\mathrm{C}(39)$ & $1.497(7)$ & $\mathrm{C}(18)-\mathrm{C}(17)-\mathrm{C}(16)$ & $107.6(5)$ \\
\hline $\mathrm{C}(32)-\mathrm{O}(1)$ & $1.377(7)$ & C(17)-C(18)-C(19) & $108.2(5)$ \\
\hline $\mathrm{N}(1 \mathrm{~S})-\mathrm{C}(3 \mathrm{~B})$ & $1.505(8)$ & $\mathrm{N}(4)-\mathrm{C}(19)-\mathrm{C}(20)$ & $125.8(4)$ \\
\hline $\mathrm{N}(1 \mathrm{~S})-\mathrm{C}(4 \mathrm{~B})$ & $1.507(8)$ & $\mathrm{N}(4)-\mathrm{C}(19)-\mathrm{C}(18)$ & $107.3(4)$ \\
\hline $\mathrm{N}(1 \mathrm{~S})-\mathrm{C}(8 \mathrm{~B})$ & $1.508(7)$ & $\mathrm{C}(20)-\mathrm{C}(19)-\mathrm{C}(18)$ & $126.9(5)$ \\
\hline $\mathrm{N}(1 \mathrm{~S})-\mathrm{C}(6 \mathrm{~B})$ & $1.522(7)$ & $\mathrm{C}(19)-\mathrm{C}(20)-\mathrm{C}(1)$ & $126.1(5)$ \\
\hline $\mathrm{O}(1)-\mathrm{C}(1 \mathrm{~B})$ & $1.425(9)$ & C(19)-C(20)-C(39) & $116.8(4)$ \\
\hline$C(1 B)-C(2 B)$ & $1.521(9)$ & $\mathrm{C}(1)-\mathrm{C}(20)-\mathrm{C}(39)$ & $117.1(5)$ \\
\hline $\mathrm{C}(2 \mathrm{~B})-\mathrm{C}(3 \mathrm{~B})$ & $1.522(9)$ & $\mathrm{O}(1)-\mathrm{C}(32)-\mathrm{C}(31)$ & $119.7(5)$ \\
\hline$C(4 B)-C(5 B)$ & $1.521(9)$ & $\mathrm{O}(1)-\mathrm{C}(32)-\mathrm{C}(27)$ & $119.6(5)$ \\
\hline$C(6 B)-C(7 B)$ & $1.505(10)$ & $\mathrm{C}(3 \mathrm{~B})-\mathrm{N}(1 \mathrm{~S})-\mathrm{C}(4 \mathrm{~B})$ & $111.5(5)$ \\
\hline \multirow[t]{3}{*}{$\mathrm{C}(8 \mathrm{~B})-\mathrm{C}(9 \mathrm{~B})$} & $1.525(9)$ & $\mathrm{C}(3 \mathrm{~B})-\mathrm{N}(1 \mathrm{~S})-\mathrm{C}(8 \mathrm{~B})$ & $106.3(4)$ \\
\hline & & $\mathrm{C}(4 \mathrm{~B})-\mathrm{N}(1 \mathrm{~S})-\mathrm{C}(8 \mathrm{~B})$ & $111.6(5)$ \\
\hline & & $\mathrm{C}(3 \mathrm{~B})-\mathrm{N}(1 \mathrm{~S})-\mathrm{C}(6 \mathrm{~B})$ & $110.3(5)$ \\
\hline $\mathrm{C}(1)-\mathrm{N}(1)-\mathrm{C}(4)$ & $105.8(4)$ & $\mathrm{C}(4 \mathrm{~B})-\mathrm{N}(1 \mathrm{~S})-\mathrm{C}(6 \mathrm{~B})$ & $106.3(4)$ \\
\hline $\mathrm{C}(6)-\mathrm{N}(2)-\mathrm{C}(9)$ & $109.4(4)$ & $\mathrm{C}(8 \mathrm{~B})-\mathrm{N}(1 \mathrm{~S})-\mathrm{C}(6 \mathrm{~B})$ & $110.8(5)$ \\
\hline $\mathrm{C}(14)-\mathrm{N}(3)-\mathrm{C}(11)$ & $106.0(4)$ & $\mathrm{C}(32)-\mathrm{O}(1)-\mathrm{C}(1 \mathrm{~B})$ & $116.0(5)$ \\
\hline $\mathrm{C}(16)-\mathrm{N}(4)-\mathrm{C}(19)$ & $108.9(4)$ & $\mathrm{O}(1)-\mathrm{C}(1 \mathrm{~B})-\mathrm{C}(2 \mathrm{~B})$ & $106.9(6)$ \\
\hline $\mathrm{N}(1)-\mathrm{C}(1)-\mathrm{C}(20)$ & $125.2(5)$ & $\mathrm{C}(1 \mathrm{~B})-\mathrm{C}(2 \mathrm{~B})-\mathrm{C}(3 \mathrm{~B})$ & $109.0(5)$ \\
\hline $\mathrm{N}(1)-\mathrm{C}(1)-\mathrm{C}(2)$ & $110.5(4)$ & $\mathrm{N}(1 \mathrm{~S})-\mathrm{C}(3 \mathrm{~B})-\mathrm{C}(2 \mathrm{~B})$ & $115.3(5)$ \\
\hline$C(20)-C(1)-C(2)$ & $124.3(5)$ & $\mathrm{N}(1 \mathrm{~S})-\mathrm{C}(4 \mathrm{~B})-\mathrm{C}(5 \mathrm{~B})$ & $114.5(5)$ \\
\hline$C(3)-C(2)-C(1)$ & $106.5(5)$ & $\mathrm{C}(7 \mathrm{~B})-\mathrm{C}(6 \mathrm{~B})-\mathrm{N}(1 \mathrm{~S})$ & $114.2(5)$ \\
\hline$C(2)-C(3)-C(4)$ & $107.6(5)$ & $\mathrm{N}(1 \mathrm{~S})-\mathrm{C}(8 \mathrm{~B})-\mathrm{C}(9 \mathrm{~B})$ & $115.3(5)$ \\
\hline
\end{tabular}


Table 4. Hydrogen-bond geometry for $\mathbf{3}\left(\AA,{ }^{\circ}\right)$

\begin{tabular}{lllll}
\hline Donor- $\mathrm{H} \cdots$ Acceptor & $D-\mathrm{H}$ & $\mathrm{H} \cdots \mathrm{A}$ & $D \cdots \mathrm{A}$ & $D-\mathrm{H} \cdots \mathrm{A}$ \\
\hline $\mathrm{N} 2-\mathrm{H} 2 \mathrm{~N} \cdots \mathrm{N} 1$ & $0.70(9)$ & $2.48(9)$ & $2.913(7)$ & $123(9)$ \\
$\mathrm{N} 2-\mathrm{H} 2 \mathrm{~N} \cdots \mathrm{N} 3$ & $0.70(9)$ & $2.43(9)$ & $2.897(7)$ & $126(8)$ \\
$\mathrm{N} 4-\mathrm{H} 4 \mathrm{~N} \cdots \mathrm{N} 1$ & $0.75(9)$ & $2.49(9)$ & $2.895(7)$ & $116(8)$ \\
$\mathrm{N} 4-\mathrm{H} 4 \mathrm{~N} \cdots \mathrm{N} 3$ & $0.75(9)$ & $2.36(8)$ & $2.905(6)$ & $131(8)$ \\
\hline
\end{tabular}

\section{Conformational analysis.}

The porphyrin 3 adopts a "close" conformation in which the amino-alkyl side arm is folded toward the core of the macrocycle, with the terminal methyl groups of the pendant triethylammonium moiety pointing toward the pyrrole rings (I, II, IV). The lateral displacement of the side arm from the porphyrin centroid is generated by a gauche/anti/anti/anti(N)/anti conformation sequence along the main chain.

The porphyrin core is quasi-planar with an average deviation of the macrocycle atoms from their least squares plane $(\Delta 24$, Table 5$)$ of only $0.065 \AA$. The larger deviations are associated with the meso-carbon $\left(C_{m}\right)$ $\mathrm{C} 10(0.106(5) \AA)$ bonded to the para substituted aryl group and with the $\mathrm{C}_{\beta}$ of the neighbouring pyrrole rings $(-0.089(6)-0.172(6) \AA)$. Likewise, the $\mathrm{C}_{\alpha}-\mathrm{C}_{\mathrm{m}}-\mathrm{C}_{\alpha}$ angle between the $\alpha$-pyrrolic and meso carbons varies only slightly (Table 5) with the smallest value and the bigger deviation at meso-C10 position again $\left(124.7(5)^{\circ}\right)$.

The overall slightly distortion results essentially in a saddle shaped conformation characterized by an alternating displacement of the pyrrole rings N(I), (III) and (IV) below and above the mean plane (Figure 24). ${ }^{9}$ A closer look of the out-of-plane distortion pattern suggests a small contribute from wave conformation involving the pyrrolic unit $\mathrm{N}$ (II) (toward which the side arm of porphyrin is bent). ${ }^{10}$ The minor deformation from planarity of porphyrin macrocycle and the different role of the pyrrolic units is also reflected by the values of the dihedral angle between the pyrrole rings and the $\mathrm{N}_{4}$-core mean plane (Table 5).

All the conformational data seem indicate that the small distortions observed for the macrocycle are essentially due to the presence and orientation of the "long" alkyl chain on the para position of a meso-aryl group. Despite the asymmetric substituent arrangement, the inner cavity shape composed by the four $\mathrm{N}$ atoms is square-like as indicated by the core elongation factor $(\Xi=0.013 \AA)$ and by the distances between the neighbouring $\mathrm{N}-\mathrm{N}$ atoms $(2.895(7)-2.913(7) \AA)$. The $\mathrm{N}-\mathrm{H}$ groups are involved in bifurcated $\mathrm{N}-\mathrm{H} \cdots(\mathrm{N}, \mathrm{N})$ intramolecular hydrogen bonds (Table 4) and result only slightly tilted out of the plane of macrocycle. 
Table 5. Selected conformational parameters for $\mathbf{3}$ (deviations and distances in $\AA$, angles in ${ }^{\circ}$ )

\begin{tabular}{llll}
\hline Core size $\otimes^{a}$ & 2.053 & $\mathrm{C}_{\alpha}-\mathrm{C}_{15}-\mathrm{C}_{\alpha}$ & $126.9(5)$ \\
Core elongation $\Xi^{b}$ & 0.013 & $\mathrm{C}_{\alpha}-\mathrm{C}_{20}-\mathrm{C}_{\alpha}$ & $126.1(5)$ \\
$\Delta 24^{c}$ & 0.065 & cis $\mathrm{C}_{\alpha}-\mathrm{N} \cdots \mathrm{N}-\mathrm{C}_{\alpha}{ }^{g}$ & 4.2 \\
$\mathrm{~N} 1 \cdots \mathrm{N} 2$ & $2.913(7)$ & trans $\mathrm{C}_{\alpha}-\mathrm{N} \cdots \mathrm{N}-\mathrm{C}_{\alpha}$ & 177.1 \\
$\mathrm{~N} 2 \cdots \mathrm{N} 3$ & $2.897(7)$ & $\varphi_{\mathrm{pyr}} \mathrm{N} 1^{h}$ & 1.89 \\
$\mathrm{~N} 3 \cdots \mathrm{N} 4$ & $2.905(6)$ & $\varphi_{\mathrm{pyr}} \mathrm{N} 2$ & 7.24 \\
$\mathrm{~N} 4 \cdots \mathrm{N} 1$ & $2.895(7)$ & $\varphi_{\mathrm{pyr}} \mathrm{N} 3$ & 3.18 \\
$\delta \mathrm{C}_{\mathrm{m}}{ }^{d}$ & 0.074 & $\varphi_{\mathrm{pyr}} \mathrm{N} 4$ & 0.93 \\
$\delta \mathrm{C}_{\alpha}{ }^{e}$ & 0.051 & $\varphi_{\mathrm{ar}} \mathrm{C} 5^{i}$ & 87.05 \\
$\delta \mathrm{C}_{\beta}{ }^{f}$ & 0.102 & $\varphi_{\mathrm{ar}} \mathrm{C} 10$ & 87.87 \\
$\mathrm{C}_{\alpha}-\mathrm{C}_{5}-\mathrm{C}_{\alpha}$ & $126.8(5)$ & $\varphi_{\mathrm{ar}} \mathrm{C} 15$ & 82.98 \\
$\mathrm{C}_{\alpha}-\mathrm{C}_{10}-\mathrm{C}_{\alpha}$ & $124.7(5)$ & $\varphi_{\mathrm{ar}} \mathrm{C} 20$ & 81.15 \\
\hline
\end{tabular}

$\mathrm{C}_{\mathrm{m}}$ (meso carbon) $=5,10,15,20 ; \mathrm{C}_{\beta}(\beta$-pyrrole position $)=2,3,7,8,12,13,17,18 ; \mathrm{C}_{\alpha}(\alpha$-pyrrole position $)=1,4,6,9,11,14,16,19 .{ }^{a}$ The core size is defined as the geometrical centre of the four nitrogen atoms. ${ }^{b}$ The core elongation parameter is defined as the difference between the vector lengths $(|\mathrm{N} 1-\mathrm{N} 2|+|\mathrm{N} 3-\mathrm{N} 4|) / 2-(|\mathrm{N} 2-\mathrm{N} 3|+|\mathrm{N} 1-\mathrm{N} 4|) / 2 .{ }^{c}$ Deviation of the 24 macrocycle atoms from their least squares plane. ${ }^{d}$ Average deviation of the $\mathrm{C}_{\mathrm{m}}$ carbon atoms from the $4 \mathrm{~N}$ plane. ${ }^{e}$ Average deviation of the $\mathrm{C}_{\alpha}$ atoms from the $4 \mathrm{~N}$ plane. ${ }^{f}$ Average deviation of the $\mathrm{C}_{\beta}$ atoms from the $4 \mathrm{~N}$ plane. ${ }^{g} \mathrm{Cis}$ $\mathrm{C}_{\alpha}-\mathrm{N}-\mathrm{N}-\mathrm{C}_{\alpha}$ dihedral angles. ${ }^{11}{ }^{h}$ Pyrrole tilt angle with the $4 \mathrm{~N}$ plane. ${ }^{i}$ Phenyl tilt angle against the $4 \mathrm{~N}$ plane.

Figure 24. Linear display of the skeletal deviations (in units of $0.001 \AA$ ) of the macrocycle atoms from the mean porphyrin plane (of 24 atom). ${ }^{10}$ The $x$ axis is not to scale.

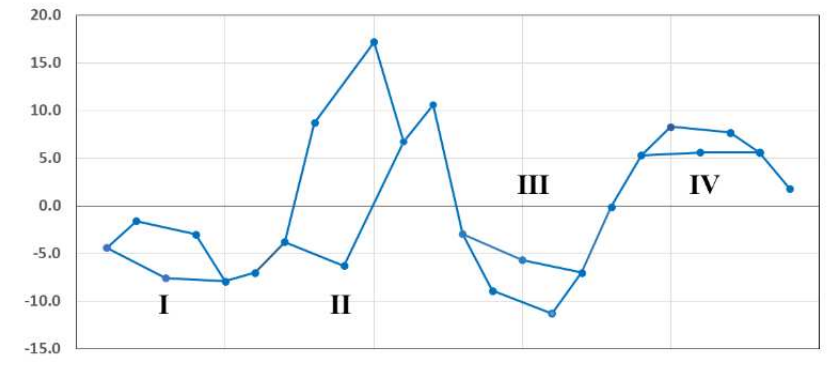

\section{References}

${ }^{1}$ T. A. D. Pinto, R. Hrdina, G. Kirsch, A. M. F. Oliveira-Campos, L. M. Rodrigues and A. P. Esteves, ARKIVOC 2012, 6, 185-193

${ }^{2}$ H. G. Brittain, Cryst. Growth Des., 2010, 10, 1990-2003

${ }^{3}$ Bruker, SAINT+, 2007, Bruker AXS Inc., Madison, Wisconsin, USA.

${ }^{4}$ Bruker, APEX II, 2009, Bruker AXS Inc., Madison, Wisconsin, USA.

${ }^{5}$ M. C. Burla, R. Caliandro, M. Camalli, B. Carrozzini, G. L. Cascarano, L. De Caro, C. Giacovazzo, G. Polidori and R. Spagna, J. Appl. Crystallogr., 2005, 38, 381-388.

${ }^{6}$ G. M. Sheldrick, Acta Crystallogr. Sect. C Struct. Chem., 2015, 71, 3-8.

${ }^{7}$ L. J. Farrugia, J. Appl. Crystallogr., 2012, 45, 849-854.

${ }^{8} \mathrm{~K}$. A. Connors: Binding constants - the measurement of molecular complex stability, John Wiley \& Sons, New York 1987, 91, 1398-1398.

${ }^{9}$ M. Gruden, S. Grubišić, A. G. Coutsolelos and S. R. Niketić, J. Mol. Struct., 2001, 595, 209-224.

${ }^{10}$ a) W. Jentzen, X.-Z. Song and J. A. Shelnutt, J. Phys. Chem. B, 1997, 101, 1684-1699; b) W. Jentzen, J.-G. Ma and J. A. Shelnutt, Biophys. J., 1998, 74, 753-763.

${ }^{11}$ A. Fidalgo-Marijuan, G. Barandika, B. Bazán, M.-K. Urtiaga and M. I. Arriortua, CrystEngComm, $2013,15,4181$. 
Electronic Supplementary Information

\section{Sensing of Diclofenac by a Porphyrin-based Artificial Receptor}

Daniela Intrieri, ${ }^{\mathrm{a}}$ Caterina Damiano, ${ }^{\mathrm{a}}$ Silvia Rizzato, ${ }^{\mathrm{a}}$ Roberto Paolesse, ${ }^{\mathrm{b}}$ Mariano Venanzi, ${ }^{\mathrm{b}}$ Donato Monti, ${ }^{* \mathrm{~b}}$ Marco Savioli ${ }^{\mathrm{b}}$, Manuela Stefanelli ${ }^{\mathrm{b}}$ and Emma Gallo. ${ }^{\mathrm{a}}$

${ }^{a}$ Department of Chemistry, University of Milan, Via C. Golgi 19, 20133 Milan (Italy). E-mail: emma.gallo@unimi.it.

${ }^{b}$ Department of Chemical Science and Technologies, University of Roma Tor Vergata, Via della Ricerca Scientifica,00133 Rome (Italy).E-mail:dmonti@uniroma2.it

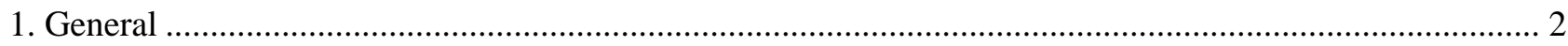

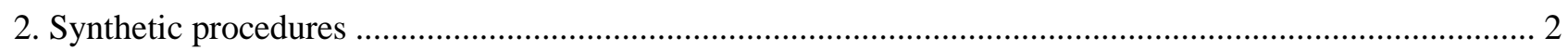

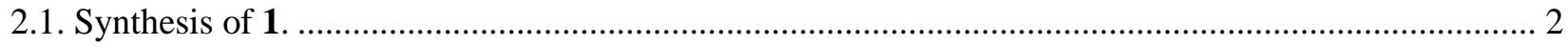

2.2. Synthesis of 2.

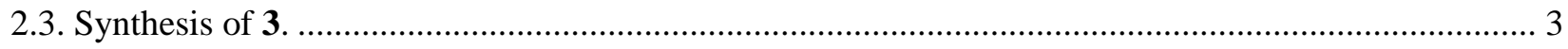

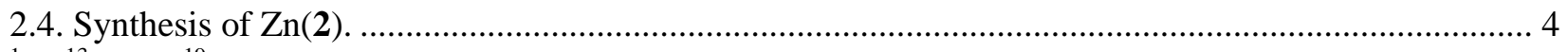

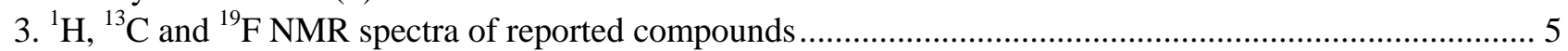

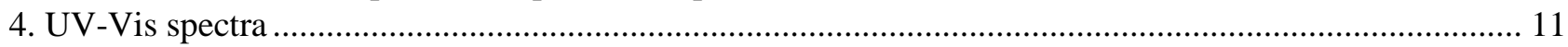

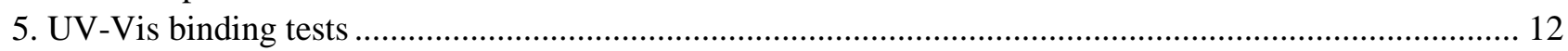

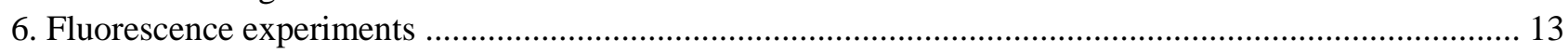

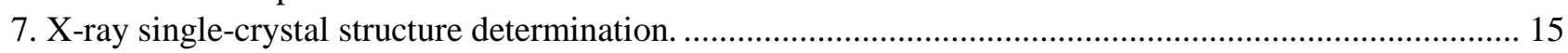

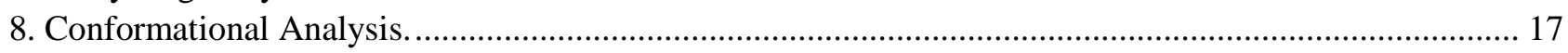

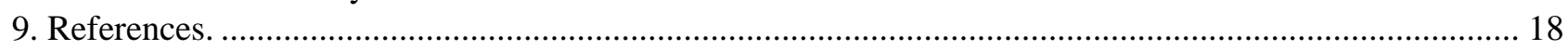




\section{General}

Unless otherwise specified, all the reactions were carried out under a nitrogen atmosphere by employing standard Schlenk techniques and magnetic stirring. Dichloromethane, chloroform, $N, N$-dimethylformamide and pyrrole were distilled over $\mathrm{CaH}_{2}$ and stored under nitrogen. Acetone was distilled over $\mathrm{K}_{2} \mathrm{CO}_{3}$ and stored under nitrogen. All the other starting materials and $\mathrm{Zn}(\mathrm{TPP})$ were commercial products which were used as received. 2-(3-Bromopropoxy)benzaldehyde ${ }^{1}$ and sodium phenylacetate ${ }^{2}$ were synthesized by methods reported in the literature or by using minor modifications of them. Solvents used for spectroscopy investigations were of Spectroscopic Grade of the highest degree of purity available and used as received. NMR spectra were recorded at room temperature on a Bruker Avance 400-DRX spectrometers, operating at $400 \mathrm{MHz}$ for ${ }^{1} \mathrm{H}$, at $100 \mathrm{MHz}$ for ${ }^{13} \mathrm{C}$ and at $376 \mathrm{MHz}$ for ${ }^{19} \mathrm{~F}$. Chemical shifts (ppm) are reported relative to TMS. The ${ }^{1} \mathrm{H}$ NMR signals of the compounds described in the following have been attributed by COSY and NOESY techniques. Assignments of the resonance in ${ }^{13} \mathrm{C}$ NMR were made using the APT pulse sequence and HSQC and HMBC techniques. UV/Vis spectra were recorded on an Agilent 8453E instrument. Elemental analyses and mass spectra were recorded in the analytical laboratories of Milan University. All spectroscopic studies were carried out at $298.0( \pm 0.5)$ K (Julabo F25 Thermostat). Steady-state Fluorescence and Resonance Light Scattering Spectroscopy studies were carried out on Fluoromax 4 (Horiba Instruments). Time-resolved Fluorescence Spectroscopy studies were carried out on LifeSpoec-ps (Edimburg Instruments), equipped with a Hamamatsu Photonics K.K. laser. X-ray data collection was performed at 150 $\mathrm{K}$ using graphite-monochromated Mo K $\alpha$ radiation $(\lambda=0.71073 \AA)$ on a Bruker ApexII CCD area-detector diffractometer equipped with an Oxford Cryosystems $\mathrm{N}_{2}$ gas blower. A $\omega$-scan was performed within the Bragg limits of $1.3<\theta<25.0^{\circ}$. Determination of the integrated intensities and unit cell refinements were performed using SAINT $^{3}$ and all absorption corrections were applied by using SADABS. ${ }^{4}$ The structures were solved by direct methods (SIR2014) ${ }^{5}$ and refined by full-matrix least squares on $\mathrm{F}^{2}$ (SHELX 2014) ${ }^{6}$ with the WINGX interface. ${ }^{7}$

\section{Synthetic procedures}

\subsection{Synthesis of 1 .}

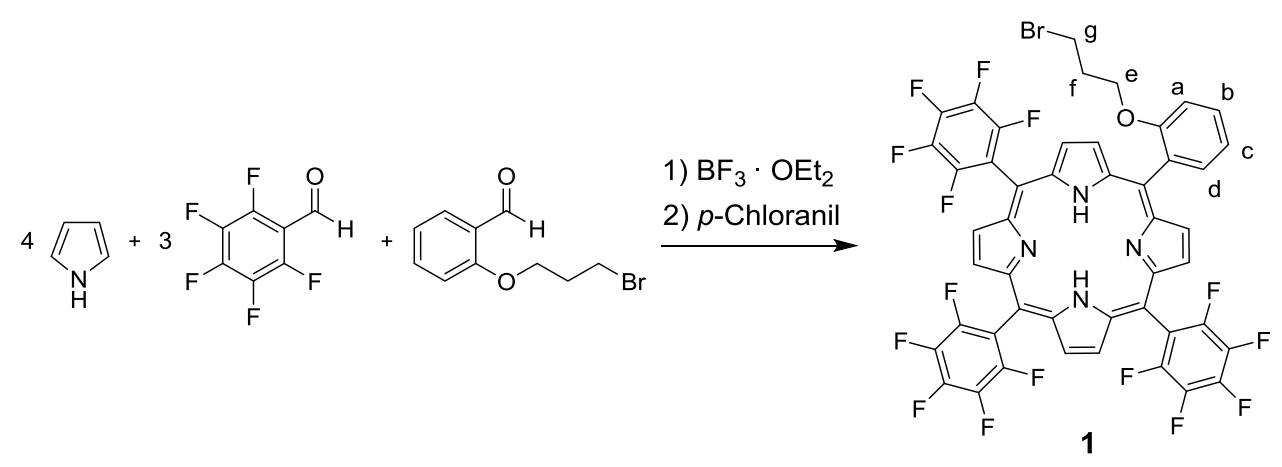

Freshly distilled pyrrole $(173 \mu \mathrm{L}, 2.50 \mathrm{mmol})$, pentafluorobenzaldehyde $(231 \mu \mathrm{L}, 1.87 \mathrm{mmol})$ and 2-(3bromopropoxy)benzaldehyde $(110 \mu \mathrm{L}, 0.62 \mathrm{mmol})$ were dissolved in dry dichloromethane $(250 \mathrm{~mL})$ in a 500 $\mathrm{mL}$ two necks round-bottom flask. The reaction mixture was shielded from ambient light and $\mathrm{BF}_{3} \cdot \mathrm{OEt}_{2}(31$ $\mu \mathrm{L}, 0.25 \mathrm{mmol}$ ) was added dropwise by a syringe. The obtained pale orange solution was stirred in the dark for 3 hours at room temperature and then tetrachloro-1,4-benzoquinone ( $p$-chloranil) $(0.615 \mathrm{~g}, 2.50 \mathrm{mmol})$ was added. The resulting solution was refluxed in air for 6 hours, the solvent evaporated to dryness under reduced pressure and the resulting black solid purified by flash column chromatography on silica gel ( $60 \mu \mathrm{m}$, eluent $n$-hexane/dichloromethane $=90: 10)$ yielding $1(20 \%$ yield $)$ as a purple solid. Elemental Analysis calc. for $\mathrm{C}_{47} \mathrm{H}_{20} \mathrm{BrF}_{15} \mathrm{~N}_{4} \mathrm{O}$ : C, 55.26; H, 1.97; N, 5.48; found: C, 55.66; H, 2.11; N, 5.31. UV-Vis, $\lambda_{\max }(\mathrm{MeOH}) / \mathrm{nm}$ $\left(\log \varepsilon_{\mathrm{M}}\right): 410$ (5.39), 506 (4.47), 536 (3.61), 582 (3.98), 643 (3.15). LR-MS (ESI): m/z $\left(\mathrm{C}_{47} \mathrm{H}_{20} \mathrm{BrF}_{15} \mathrm{~N}_{4} \mathrm{O}\right.$ $\left.[\mathrm{M}+\mathrm{H}]^{+}\right)$calcd. 1020.06; found 1021.2. ${ }^{1} \mathrm{H} \mathrm{NMR}\left(400 \mathrm{MHz}, \mathrm{CDCl}_{3}, 298 \mathrm{~K}\right) \delta: 8.97(\mathrm{~d}, J=4.7 \mathrm{~Hz}, 2 \mathrm{H}$, $\left.\mathrm{H}_{\text {ppyrr }}\right), 8.91\left(\mathrm{~s}, 4 \mathrm{H}, \mathrm{H}_{\text {ppyrr }}\right), 8.84\left(\mathrm{~d}, J=4.7 \mathrm{~Hz}, 2 \mathrm{H}, \mathrm{H}_{\text {ppyrr }}\right), 8.08\left(\mathrm{dd}, J_{o}=7.4 \mathrm{~Hz}, J_{m}=1.5 \mathrm{~Hz}, 1 \mathrm{H}, \mathrm{H}_{\mathrm{d}}\right), 7.83$ $\left(\mathrm{t}, J=8.1 \mathrm{~Hz}, 1 \mathrm{H}, \mathrm{H}_{\mathrm{b}}\right), 7.44\left(\mathrm{t}, J=7.6 \mathrm{~Hz}, 1 \mathrm{H}, \mathrm{H}_{\mathrm{c}}\right), 7.38\left(\mathrm{~d}, J=8.1 \mathrm{~Hz}, 1 \mathrm{H}, \mathrm{H}_{\mathrm{a}}\right), 4.10\left(\mathrm{t}, J=5.4 \mathrm{~Hz}, 2 \mathrm{H}, \mathrm{H}_{\mathrm{e}}\right)$, 
$2.26\left(\mathrm{t}, J=6.0 \mathrm{~Hz}, 2 \mathrm{H}, \mathrm{H}_{\mathrm{g}}\right), 1.46\left(\mathrm{~m}, 2 \mathrm{H}, \mathrm{H}_{\mathrm{f}}\right),-2.81\left(\mathrm{~s}, 2 \mathrm{H}, \mathrm{NH} H_{p y r}\right) .{ }^{13} \mathrm{C} \mathrm{NMR}\left(100 \mathrm{MHz}, \mathrm{CDCl}_{3}, 298 \mathrm{~K}\right) \delta$ : 158.3, 147.8, 145.4, 143.4, 140.9, 138.8, 136.3, 135.6, 130.6, 129.8 (2 signals overlapped), 119.9, 119.4, $116.0,117.7,102.8,101.9,65.1,31.3,29.8$. Six quaternary carbon atoms were not detected. ${ }^{19} \mathrm{~F}$ NMR $(376$ $\left.\mathrm{MHz}, \mathrm{CDCl}_{3}, 298 \mathrm{~K}\right) \delta:-136.9,-152.1,-162.0$.

\subsection{Synthesis of 2 .}
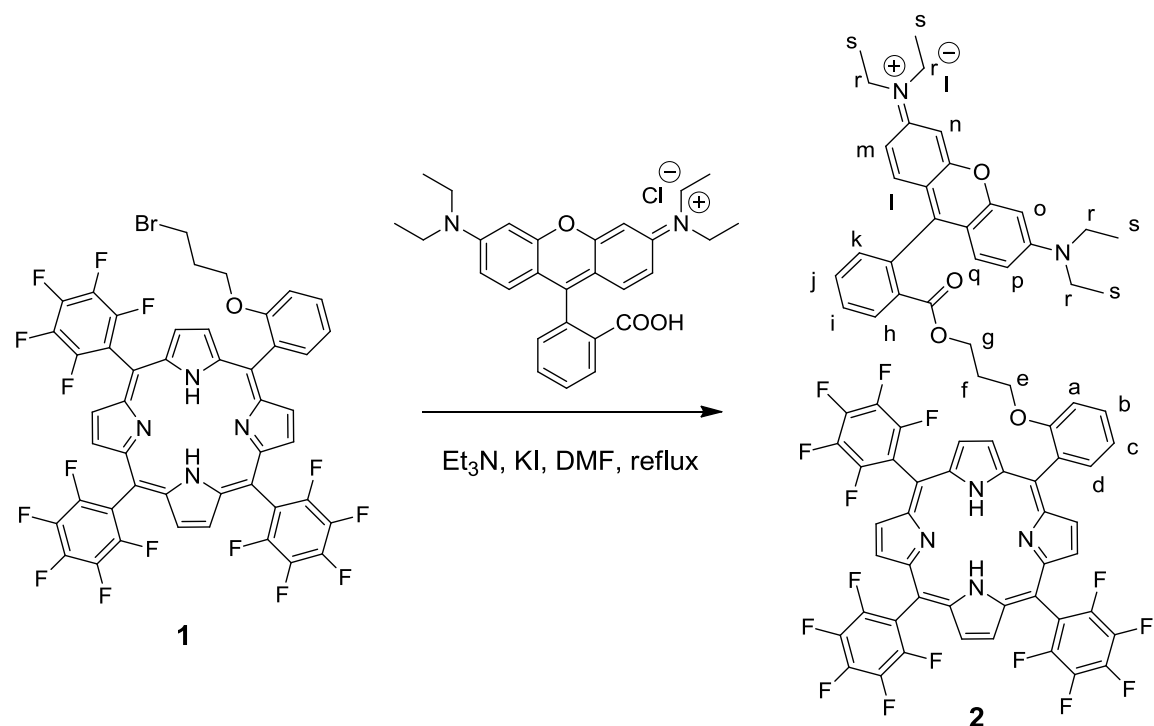

Rhodamine B $(0.200 \mathrm{~g}, 0.418 \mathrm{mmol})$, triethylamine $(58.0 \mu \mathrm{L}, 0.418 \mathrm{mmol})$ and $\mathrm{KI}(0.069 \mathrm{~g}, 0.418 \mathrm{mmol})$ were added to a dry DMF $(25 \mathrm{~mL})$ solution of $1(0.171 \mathrm{~g}, 0.167 \mathrm{mmol})$. The dark purple mixture was refluxed under stirring for 10 hours, then the solvent was evaporated to dryness under reduced pressure and the residue purified by flash column chromatography on silica gel $(60 \mu \mathrm{m}$, eluent: gradient, from dichloromethane to $3 \%$ methanol in dichloromethane) yielding 2 (45\% yield) as a dark purple solid (which resulted pink in solution). Elemental Analysis calc. for $\mathrm{C}_{75} \mathrm{H}_{50} \mathrm{~F}_{15} \mathrm{IN}_{6} \mathrm{O}_{4}: \mathrm{C}, 59.61 ; \mathrm{H}, 3.34 ; \mathrm{N}, 5.56$; found: $\mathrm{C}$, 59.93; H, 3.13; N, 5.83. UV-Vis, $\lambda_{\max }(\mathrm{MeOH}) / \mathrm{nm}\left(\log \varepsilon_{\mathrm{M}}\right): 412$ (5.67), 512 (4.82), 559 (5.24), 638 (3.18). LR-MS (ESI): $\mathrm{m} / \mathrm{z}\left(\mathrm{C}_{75} \mathrm{H}_{50} \mathrm{~F}_{15} \mathrm{~N}_{6} \mathrm{O}_{4}{ }^{+}[\mathrm{M}]^{+}\right)$calcd. 1383.36; found 1383.6. ${ }^{1} \mathrm{H}$ NMR (400 MHz, $\left.\mathrm{CDCl}_{3}, 298 \mathrm{~K}\right)$ $\delta: 8.87\left(\mathrm{~m}, 6 \mathrm{H}, \mathrm{H}_{\text {ppyrr }}\right), 8.73\left(\mathrm{~d}, J=4.3 \mathrm{~Hz}, 2 \mathrm{H}, \mathrm{H}_{\text {ppyrr }}\right), 8.00\left(\mathrm{dd}, J_{o}=7.4 \mathrm{~Hz}, J_{m}=1.6 \mathrm{~Hz}, 1 \mathrm{H}, \mathrm{H}_{\mathrm{d}}\right), 7.78(\mathrm{~m}$, $2 \mathrm{H}, \mathrm{H}_{\mathrm{b}}$ and $\left.\mathrm{H}_{\mathrm{h}}\right), 7.63\left(\mathrm{~m}, 1 \mathrm{H}, \mathrm{H}_{\mathrm{j}}\right), 7.40\left(\mathrm{t}, J=7.4 \mathrm{~Hz}, 2 \mathrm{H}, \mathrm{H}_{\mathrm{c}}\right.$ and $\left.\mathrm{H}_{\mathrm{i}}\right), 7.31\left(\mathrm{~m}, 1 \mathrm{H}, \mathrm{H}_{\mathrm{a}}\right), 7.14\left(\mathrm{~m}, 1 \mathrm{H}, \mathrm{H}_{\mathrm{k}}\right)$, $6.83\left(\mathrm{~d}, J=9.9 \mathrm{~Hz}, 2 \mathrm{H}, \mathrm{H}_{\mathrm{l}}\right.$ and $\left.\mathrm{H}_{\mathrm{q}}\right), 6.64\left(\mathrm{~m}, 4 \mathrm{H}, \mathrm{H}_{\mathrm{m}}, \mathrm{H}_{\mathrm{n}}, \mathrm{H}_{\mathrm{o}}\right.$ and $\left.\mathrm{H}_{\mathrm{p}}\right), 3.87\left(\mathrm{t}, J=5.5 \mathrm{~Hz}, 2 \mathrm{H}, \mathrm{H}_{\mathrm{e}}\right), 3.43(\mathrm{~m}$, $\left.8 \mathrm{H}, \mathrm{H}_{\mathrm{r}}\right), 3.17\left(\mathrm{t}, J=6.1 \mathrm{~Hz}, 2 \mathrm{H}, \mathrm{H}_{\mathrm{g}}\right), 1.13\left(\mathrm{~m}, 14 \mathrm{H}, \mathrm{H}_{\mathrm{f}}\right.$ and $\left.\mathrm{H}_{\mathrm{s}}\right),-2.84\left(\mathrm{~s}, 2 \mathrm{H}, \mathrm{N} H_{p y r r}\right) .{ }^{13} \mathrm{C} \mathrm{NMR}(100 \mathrm{MHz}$, $\left.\mathrm{CDCl}_{3}, 298 \mathrm{~K}\right) \delta: 164.4,158.6,158.1,157.5,155.3,147.8,145.3,143.4,140.9,138.8,135.8,133.2,132.9$, 131.3, 131.1, 131.0, 130.9, 130.1, 129.62, 129.56, 120.0, 119.4, 116.3, 114.2, 113.3, 112.0, 102.7, 96.3, 64.6, 61.9, 46.1, 43.5, 41.3, 36.0, 29.1 27.8, 12.5. ${ }^{19} \mathrm{~F}$ NMR (376 MHz, $\left.\mathrm{CDCl}_{3}, 298 \mathrm{~K}\right) \delta:-137.0,-140.38,-140.89$, $-152.0,-161.8$.

\subsection{Synthesis of 3 .}
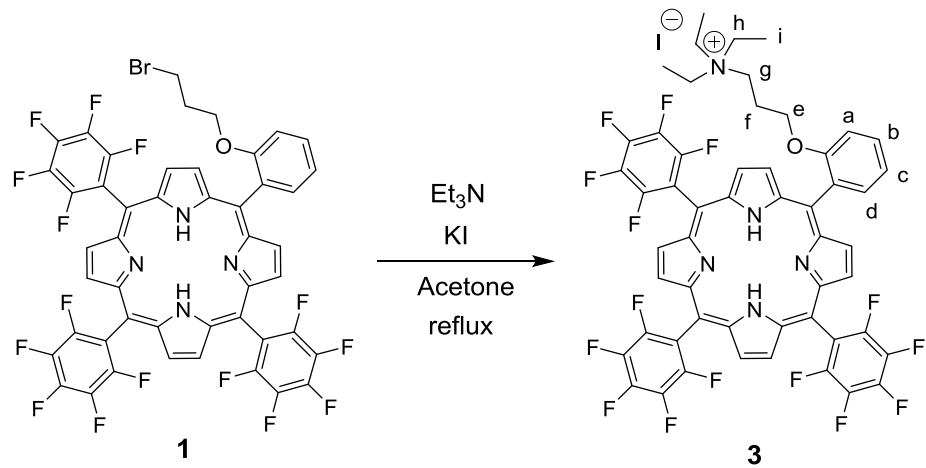

Triethylamine $(0.172 \mathrm{~mL}, 1.24 \mathrm{mmol})$ and $\mathrm{KI}(0.207 \mathrm{~g}, 1.24 \mathrm{mmol})$ were added to a dry acetone $(15 \mathrm{~mL})$ solution of $1(0.127 \mathrm{~g}, 0.124 \mathrm{mmol})$. The dark brown mixture was refluxed under stirring for 6 hours, then 
the solvent evaporated to dryness under reduced pressure and the resulting solid purified by flash column chromatography on silica gel $(60 \mu \mathrm{m}$, eluent: gradient, from dichloromethane to $3 \%$ methanol in dichloromethane) yielding 3 (55\% yield) as a dark red solid. Elemental Analysis calc. for $\mathrm{C}_{53} \mathrm{H}_{35} \mathrm{~F}_{15} \mathrm{IN}_{5} \mathrm{O}$ : $\mathrm{C}$, 54.42; H, 3.02; N, 5.99; found: C, 54.23; H, 3.11; N, 6.06. UV-Vis, $\lambda_{\max }\left(\mathrm{CH}_{2} \mathrm{Cl}_{2}\right) / \mathrm{nm}\left(\log \varepsilon_{\mathrm{M}}\right): 413(5.49)$, 507 (4.28), 536 (3.47), 583 (3.80), 636 (3.14). $\lambda_{\max }(\mathrm{MeOH}) / \mathrm{nm}\left(\log \varepsilon_{\mathrm{M}}\right): 409$ (5.49), 505 (4.27), 535 (3.39), 580 (3.76), 635 (2.96). LR-MS (ESI): $\mathrm{m} / \mathrm{z}\left(\mathrm{C}_{53} \mathrm{H}_{35} \mathrm{~F}_{15} \mathrm{~N}_{5} \mathrm{O}^{+}[\mathrm{M}]^{+}\right)$calcd. 1042.26; found 1042.4. ${ }^{1} \mathrm{H}$ NMR $\left(400 \mathrm{MHz}, \mathrm{CDCl}_{3}, 298 \mathrm{~K}\right) \delta: 8.96\left(\mathrm{~m},, 6 \mathrm{H}, \mathrm{H}_{\beta \mathrm{pyrr}}\right), 8.89\left(\mathrm{~d}, J=4.5 \mathrm{~Hz}, 2 \mathrm{H}, \mathrm{H}_{\beta \mathrm{pyrr}}\right), 8.22\left(\mathrm{dd}, J_{o}=7.6 \mathrm{~Hz}, J_{m}=\right.$ $\left.1.5 \mathrm{~Hz}, 1 \mathrm{H}, \mathrm{H}_{\mathrm{d}}\right), 7.86\left(\mathrm{t}, J=8.0 \mathrm{~Hz}, 1 \mathrm{H}, \mathrm{H}_{\mathrm{b}}\right), 7.54\left(\mathrm{t}, J=7.6 \mathrm{~Hz}, 1 \mathrm{H}, \mathrm{H}_{\mathrm{c}}\right), 7.34\left(\mathrm{~d}, J=8.0 \mathrm{~Hz}, 1 \mathrm{H}, \mathrm{H}_{\mathrm{a}}\right), 3.96$ $\left(\mathrm{t}, J=4.8 \mathrm{~Hz}, 2 \mathrm{H}, \mathrm{H}_{\mathrm{e}}\right), 1.29\left(\mathrm{~m}, 2 \mathrm{H}, \mathrm{H}_{\mathrm{f}}\right), 0.97\left(\mathrm{q}, J=6.9 \mathrm{~Hz}, 6 \mathrm{H}, \mathrm{H}_{\mathrm{h}}\right), 0.6\left(\mathrm{~m}, 2 \mathrm{H}, \mathrm{H}_{\mathrm{g}}\right),-1.26(\mathrm{t}, J=6.9 \mathrm{~Hz}$, $\left.9 \mathrm{H}, \mathrm{H}_{\mathrm{i}}\right),-2.94\left(\mathrm{~s}, 2 \mathrm{H}, \mathrm{N} H_{\text {pyrr }}\right) .{ }^{13} \mathrm{C} \mathrm{NMR}\left(100 \mathrm{MHz}, \mathrm{CDCl}_{3}, 298 \mathrm{~K}\right) \delta: 162.8,158.5,148.5,145.4,140.0$, $136.4,134.2,131.7,130.8,121.5,119.8,114.5,103.3,65.8,52.7,51.5,22.4,5.2$. Eight quaternary carbon atoms were not detected. ${ }^{19} \mathrm{~F}$ NMR $\left(376 \mathrm{MHz}, \mathrm{CDCl}_{3}, 298 \mathrm{~K}\right) \delta:-137.8,-151.0,-161.0$.

\subsection{Synthesis of $\mathrm{Zn}(2)$.}
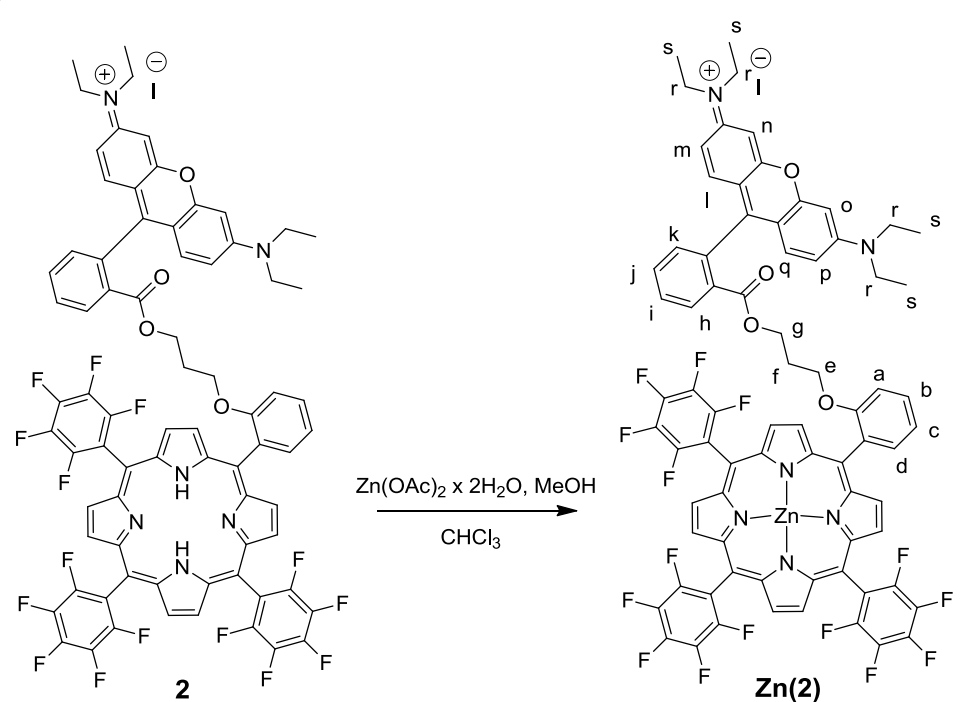

A dry $\mathrm{CH}_{3} \mathrm{OH}(13.50 \mathrm{~mL})$ solution of $\mathrm{Zn}(\mathrm{OAc})_{2} \cdot 2 \mathrm{H}_{2} \mathrm{O}(72 \mathrm{mg}, 0.33 \mathrm{mmol})$ was added to a $\mathrm{CHCl}_{3}(6.50 \mathrm{~mL})$ solution of 2 ( $49 \mathrm{mg}, 0.033 \mathrm{mmol})$ in a $50 \mathrm{~mL}$ dried Schlenk. The mixture was refluxed under stirring for 2 hours, then the solvent was evaporated to dryness under reduced pressure and $15 \mathrm{~mL} \mathrm{of} \mathrm{CH}_{2} \mathrm{Cl}_{2}$ was added to the residue. The organic phase was washed with water $(3 \times 15 \mathrm{~mL})$, dried over $\mathrm{NaSO}_{4}$ and the filtrate was evaporated to dryness under reduced pressure yielding $\mathrm{Zn}(2)$ in a quantitative yield. Elemental Analysis: calc. for $\mathrm{C}_{75} \mathrm{H}_{48} \mathrm{~F}_{15} \mathrm{IN}_{6} \mathrm{O}_{4} \mathrm{Zn}: \mathrm{C}, 62.23 ; \mathrm{H}, 3.34 ; \mathrm{N}, 5.81$; found: $\mathrm{C}, 62.40 ; \mathrm{H}, 3.71 ; \mathrm{N}, 5.62$. UV-Vis, $\lambda_{\max }$ $\left(\mathrm{CH}_{2} \mathrm{Cl}_{2}\right) / \mathrm{nm}\left(\log \varepsilon_{\mathrm{M}}\right): 419$ (5.05), 560 (4.99). $\lambda_{\max }(\mathrm{MeOH}) / \mathrm{nm}\left(\log \varepsilon_{\mathrm{M}}\right): 418(5.01), 561(4.83)$. LR-MS (ESI): $\mathrm{m} / \mathrm{z}\left(\mathrm{C}_{75} \mathrm{H}_{48} \mathrm{~F}_{15} \mathrm{~N}_{6} \mathrm{O}_{4} \mathrm{Zn}^{+}[\mathrm{M}]^{+}\right)$calcd. 1445.28; found 1446.67. ${ }^{1} \mathrm{H}$ NMR $\left(400 \mathrm{MHz}, \mathrm{CDCl}_{3}, 298 \mathrm{~K}\right): \delta$ $8.91\left(\mathrm{~m}, 6 \mathrm{H}, \mathrm{H}_{\beta \text { pyrr }}\right), 8.75\left(\mathrm{~s}, 2 \mathrm{H}, \mathrm{H}_{\beta \mathrm{pyrr}}\right), 8.14\left(\mathrm{~d}, J=6.7 \mathrm{~Hz}, 1 \mathrm{H}, \mathrm{H}_{\mathrm{h}}\right), 7.76\left(\mathrm{t}, J=7.6 \mathrm{~Hz}, 1 \mathrm{H}, \mathrm{H}_{\mathrm{j}}\right), 7.44(\mathrm{br}$ s, $2 \mathrm{H}, \mathrm{H}_{\mathrm{i}}$ and $\mathrm{H}_{\mathrm{a}}$ ), 7.26 (br s, $\mathrm{H}_{\mathrm{k}}$ and $\mathrm{H}_{\mathrm{c}}$ ), $7.13\left(\right.$ br s, $\left.1 \mathrm{H}, \mathrm{H}_{\mathrm{b}}\right), 6.88\left(\mathrm{~d}, J=7.4 \mathrm{~Hz}, 1 \mathrm{H}, \mathrm{H}_{\mathrm{d}}\right), 6.53\left(\mathrm{~s}, 2 \mathrm{H}, \mathrm{H}_{1}\right.$ and $\left.\mathrm{H}_{\mathrm{q}}\right), 6.41\left(\mathrm{~m}, 4 \mathrm{H}, \mathrm{H}_{\mathrm{m}}, \mathrm{H}_{\mathrm{n}}, \mathrm{H}_{\mathrm{o}}\right.$ and $\left.\mathrm{H}_{\mathrm{p}}\right), 3.48\left(\mathrm{~s}, 2 \mathrm{H}, \mathrm{H}_{\mathrm{e}}\right), 3.38\left(\mathrm{br} \mathrm{s}, 8 \mathrm{H}, \mathrm{H}_{\mathrm{r}}\right), 1.28$ (solvent overlap $\mathrm{H}_{\mathrm{g}}$ ), $1.12(\mathrm{~s}$, $\left.12 \mathrm{H}, \mathrm{H}_{\mathrm{s}}\right), 0.62\left(\mathrm{~s}, 2 \mathrm{H}, \mathrm{H}_{\mathrm{f}}\right) .{ }^{13} \mathrm{C} \mathrm{NMR}\left(100 \mathrm{MHz}, \mathrm{CDCl}_{3}, 298 \mathrm{~K}\right): \delta 158.8,157.9,157.3,155.3,151.3,150.1$, 149.8, 149.6, 148.3, 145.0, 135.1, 133.6, 132.7, 132.5, 132.4, 131.6, 131.2, 130.7, 130.4, 129.8, 129.7, 120.8, 114.0, 113.0, 103.1, 102.3, 96.4, 66.1, 61.8, 46.1, 29.8, 27.2, 12.6. eight quaternary carbon atoms were not detected. ${ }^{19} \mathrm{~F}$ NMR $\left(376 \mathrm{MHz}, \mathrm{CDCl}_{3}, 298 \mathrm{~K}\right): \delta-136.7,-137.7,140.3,141.3,152.4,-153.1,-162.3$ to 163.0. 


\section{3. ${ }^{1} \mathrm{H},{ }^{13} \mathrm{C}$ and ${ }^{19} \mathrm{~F}$ NMR spectra of reported compounds}

Figure 1. ${ }^{1} \mathrm{H}$ NMR spectrum (400 MHz, $\mathrm{CDCl}_{3}, 298 \mathrm{~K}$ ) of porphyrin 1

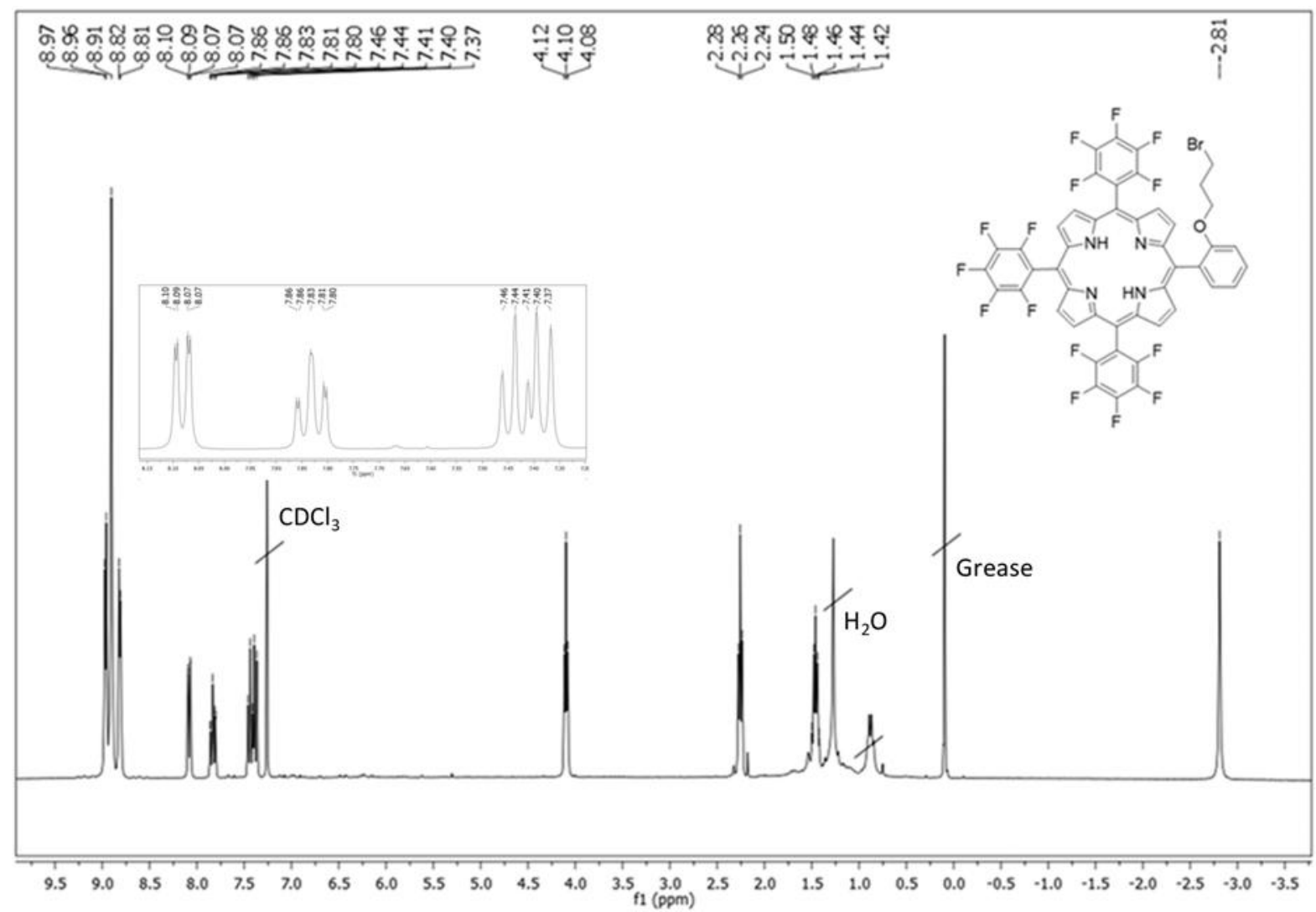

Figure 2. ${ }^{13} \mathrm{C}$ NMR spectrum $\left(100 \mathrm{MHz}, \mathrm{CDCl}_{3}, 298 \mathrm{~K}\right)$ of porphyrin $\mathbf{1}$

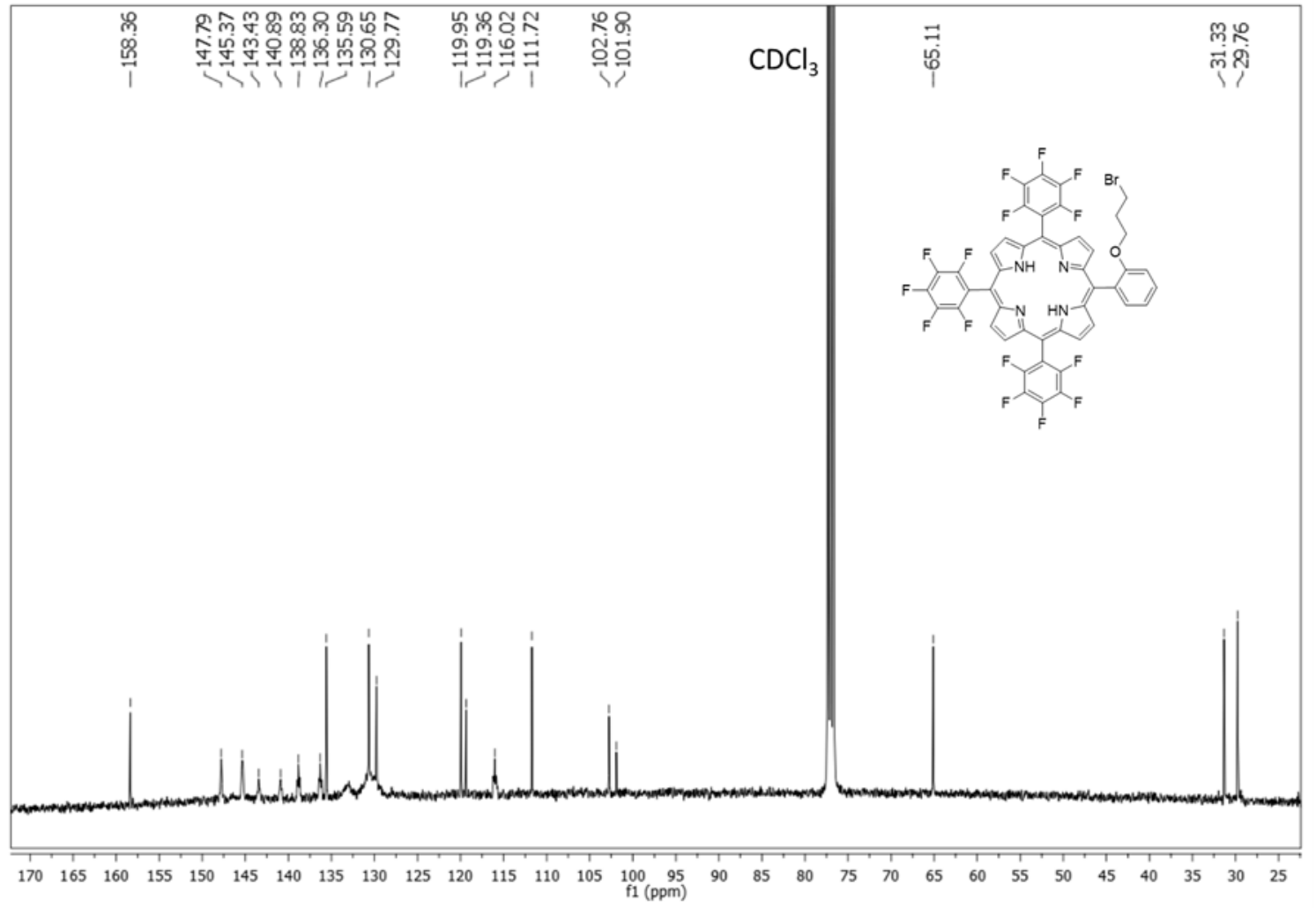


Figure 3. ${ }^{19} \mathrm{~F}$ NMR spectrum (376 MHz, $\mathrm{CDCl}_{3}, 298 \mathrm{~K}$ ) of porphyrin 1

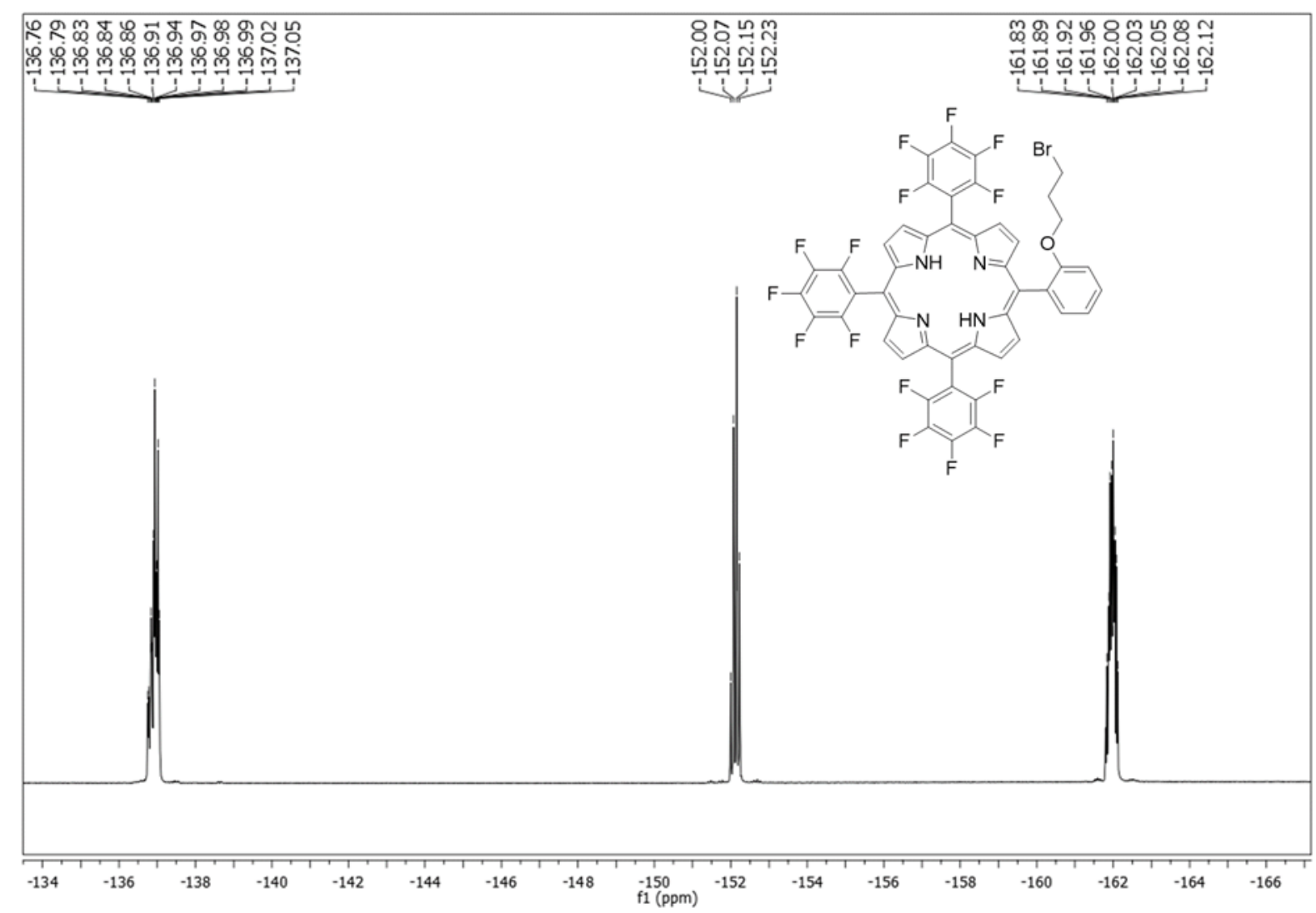

Figure 4. ${ }^{1} \mathrm{H}$ NMR spectrum $\left(400 \mathrm{MHz}, \mathrm{CDCl}_{3}, 298 \mathrm{~K}\right.$ ) of porphyrin 2

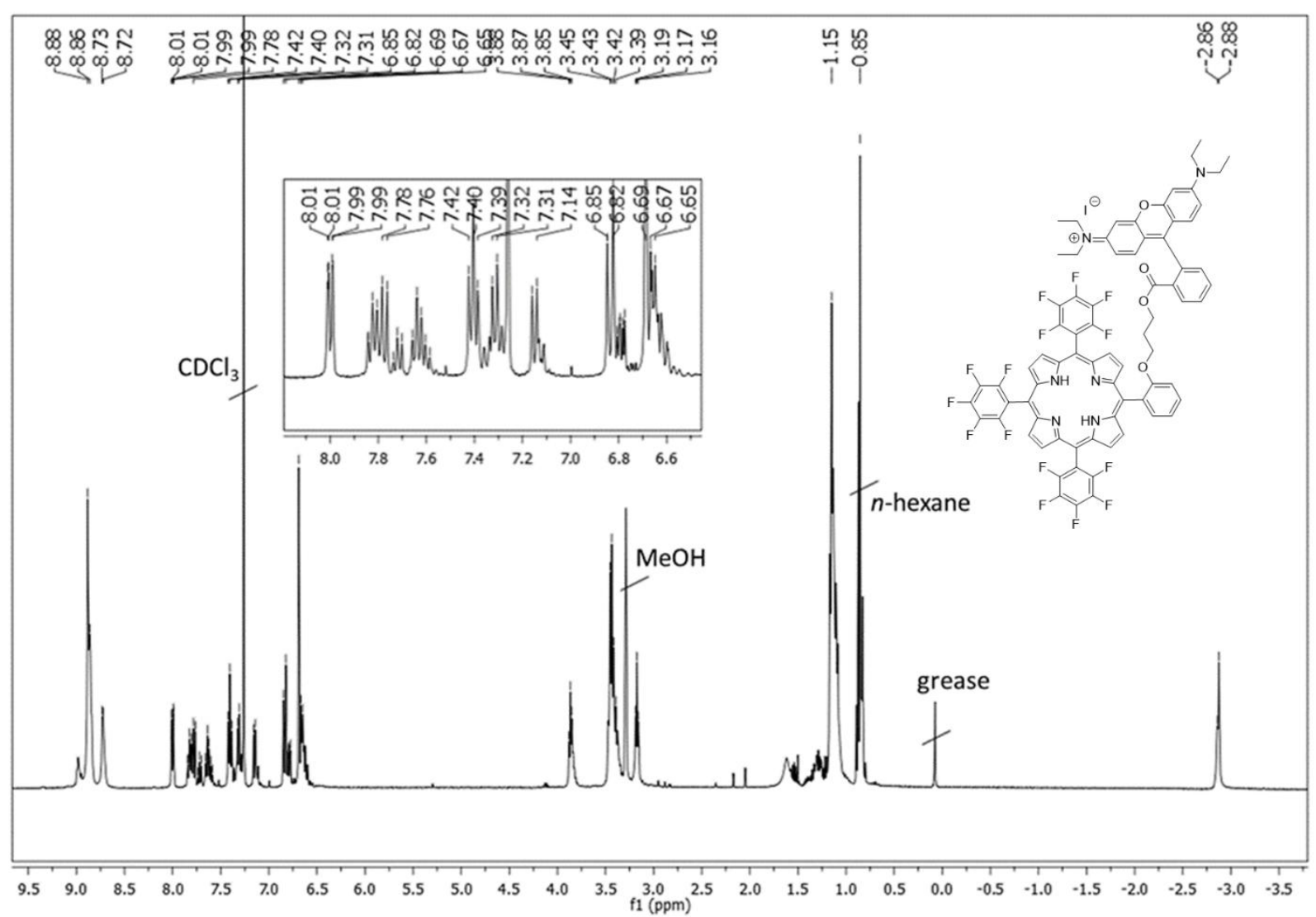


Figure 5. ${ }^{13} \mathrm{C}$ NMR spectrum (100 MHz, $\left.\mathrm{CDCl}_{3}, 298 \mathrm{~K}\right)$ of porphyrin 2

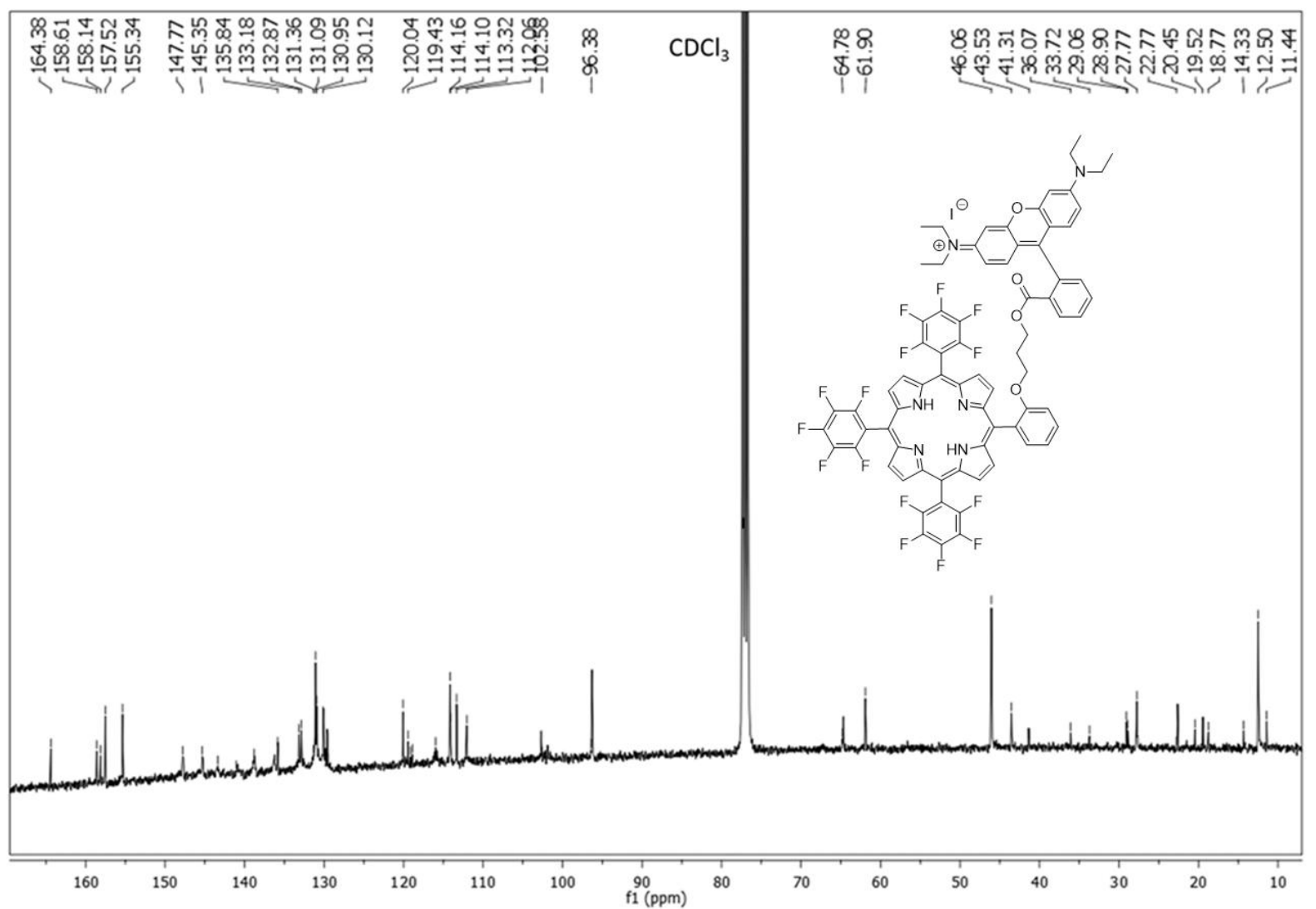

Figure 6. ${ }^{19} \mathrm{~F}$ NMR spectrum $\left(376 \mathrm{MHz}, \mathrm{CDCl}_{3}, 298 \mathrm{~K}\right)$ of porphyrin 2

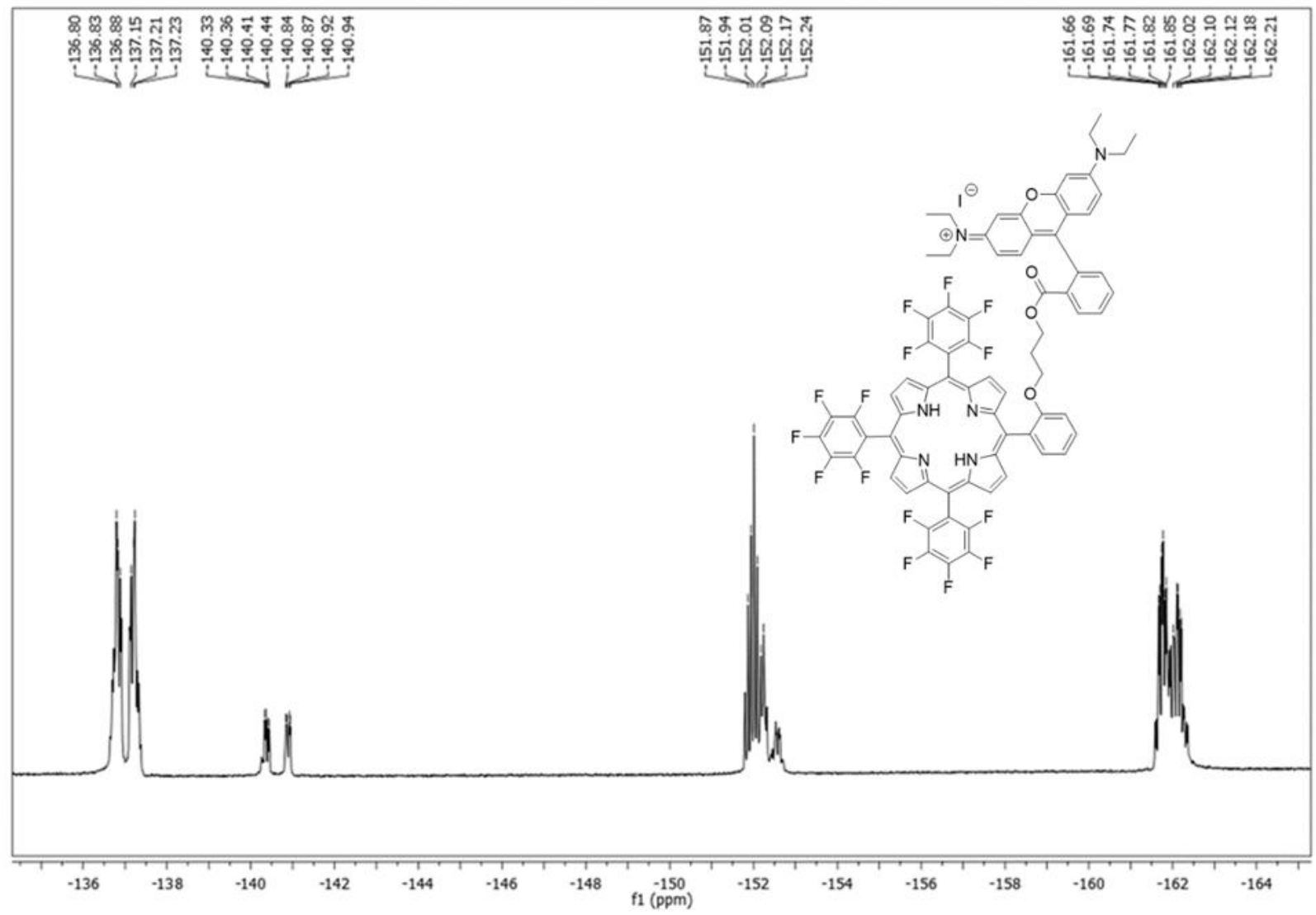


Figure 7. ${ }^{1} \mathrm{H}$ NMR spectrum (400 MHz, $\mathrm{CDCl}_{3}, 298 \mathrm{~K}$ ) of porphyrin 3

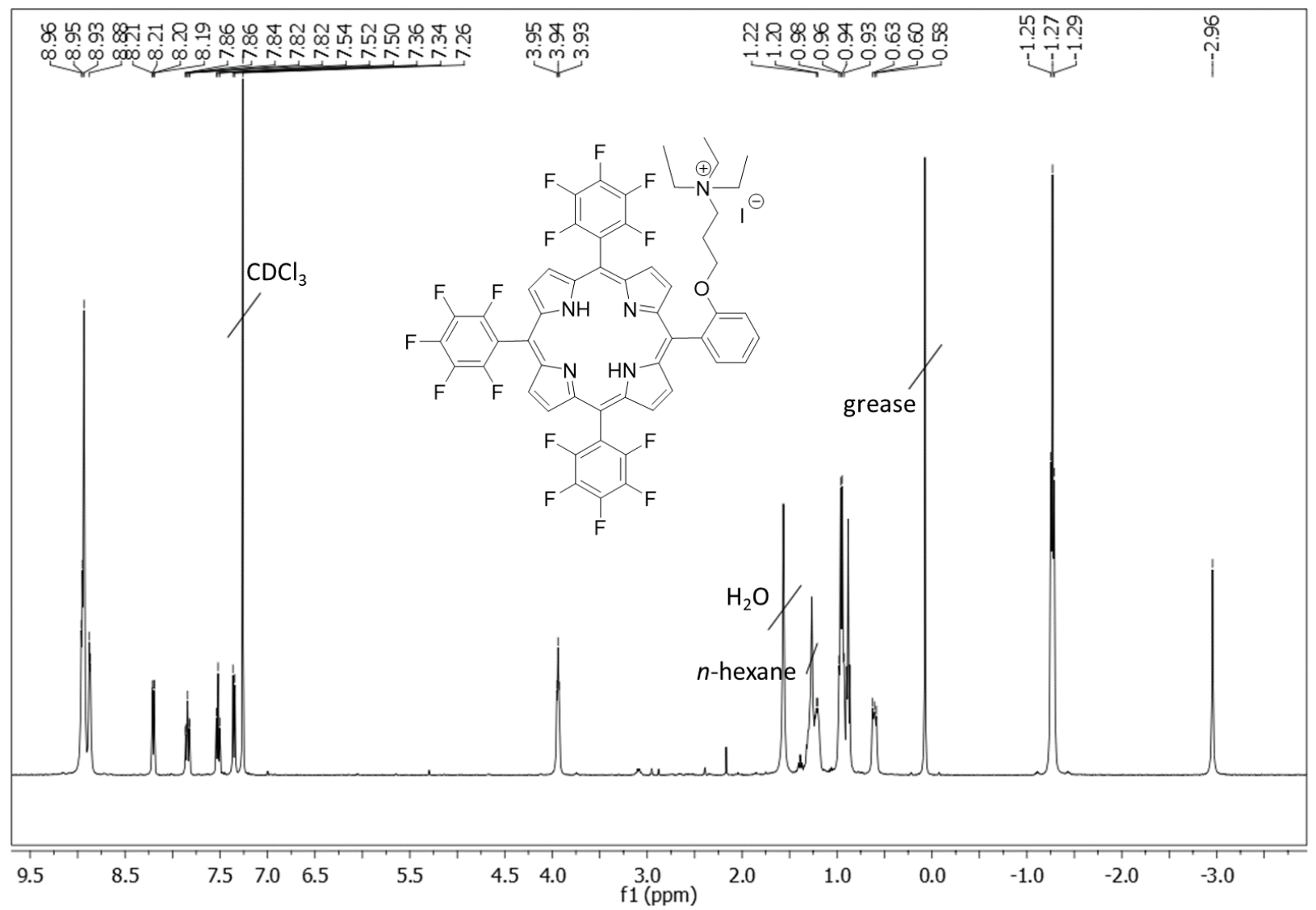

Figure $8 .{ }^{13} \mathrm{C}$ NMR spectrum $\left(100 \mathrm{MHz}, \mathrm{CDCl}_{3}, 298 \mathrm{~K}\right)$ of porphyrin 3

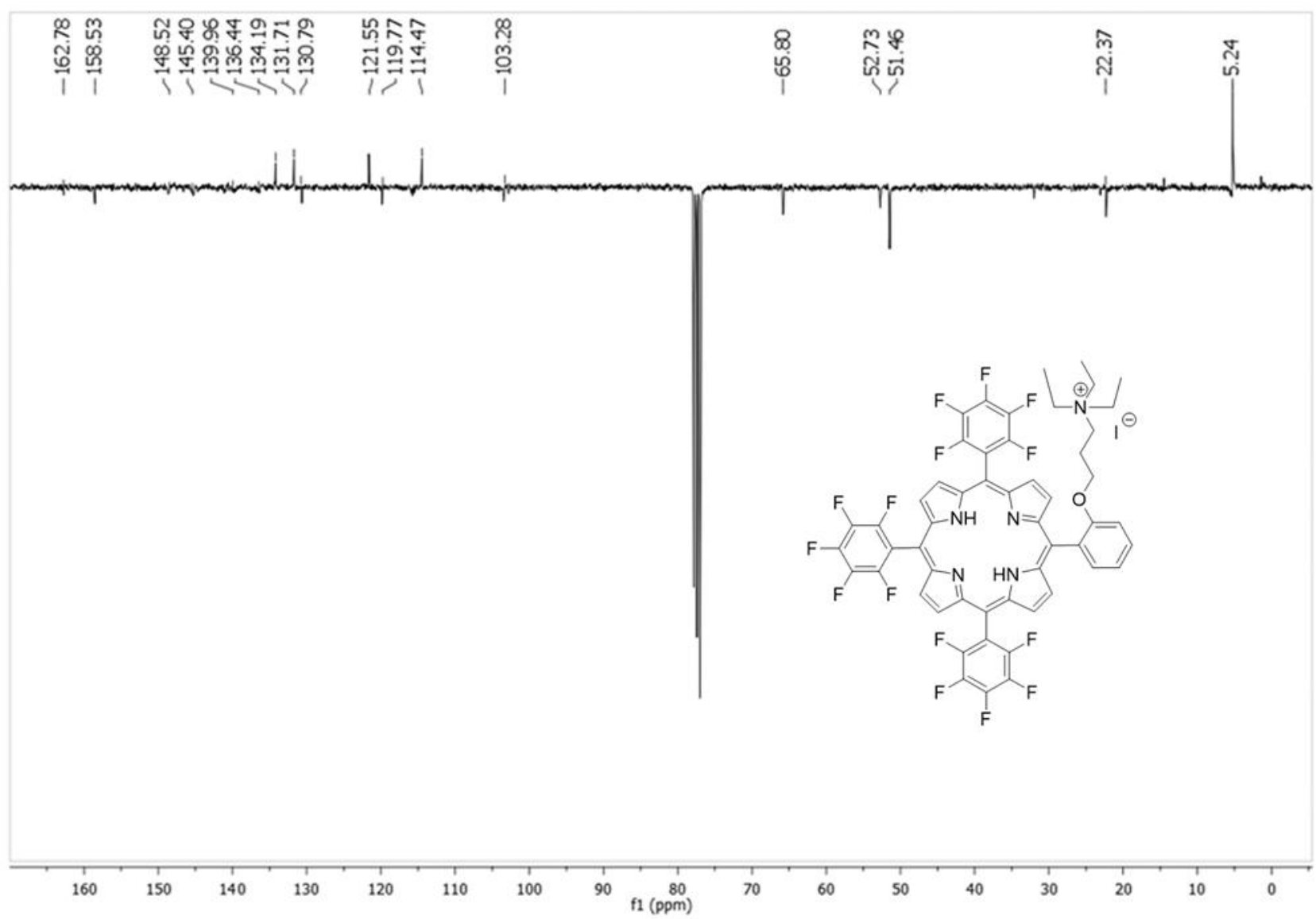


Figure 9. ${ }^{19} \mathrm{~F}$ NMR spectrum (376 MHz, $\mathrm{CDCl}_{3}, 298 \mathrm{~K}$ ) of porphyrin 3

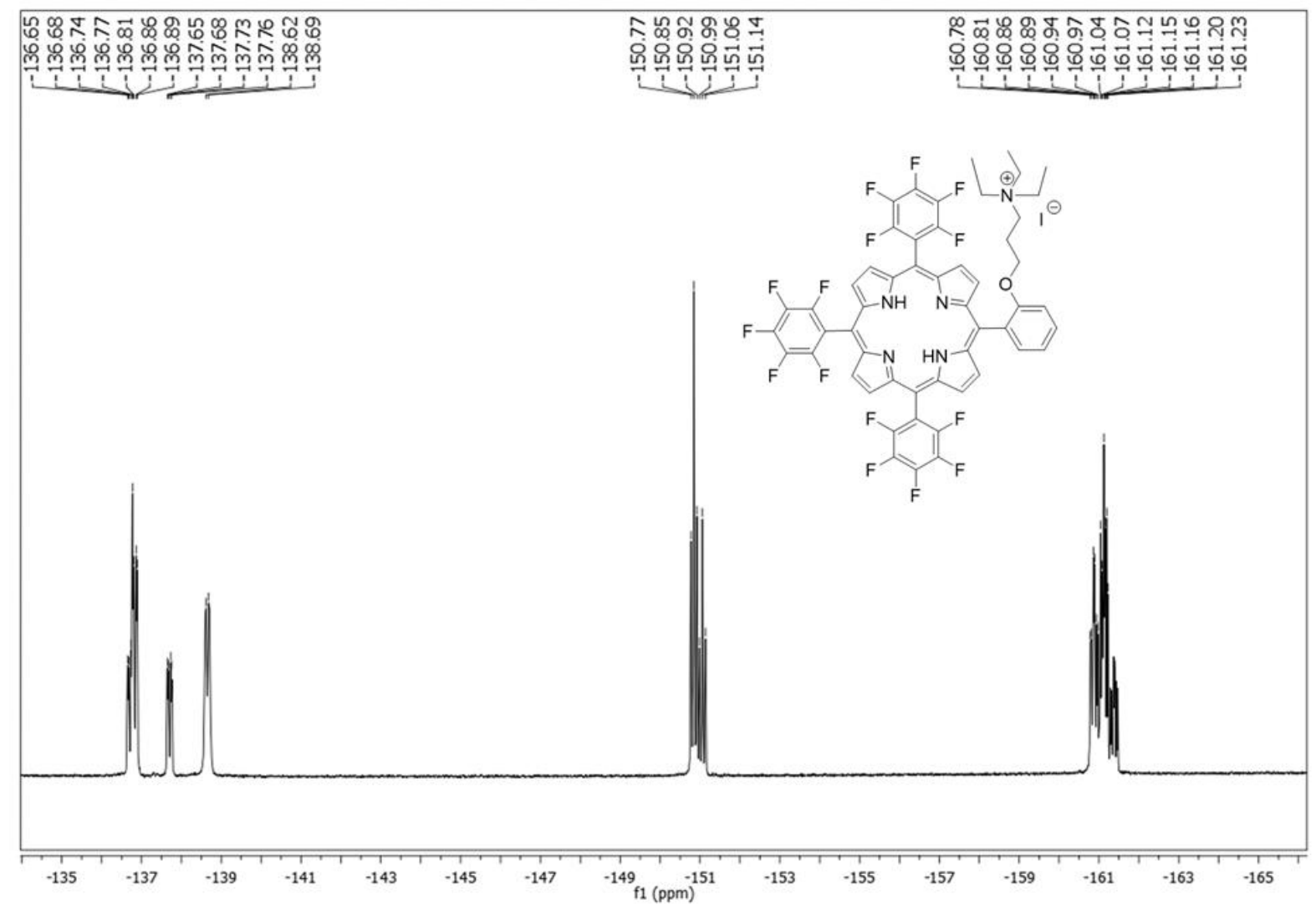

Figure 10. ${ }^{1} \mathrm{H}$ NMR spectrum (400 MHz, $\mathrm{CDCl}_{3}, 298 \mathrm{~K}$ ) of porphyrin $\mathrm{Zn}(\mathbf{2})$

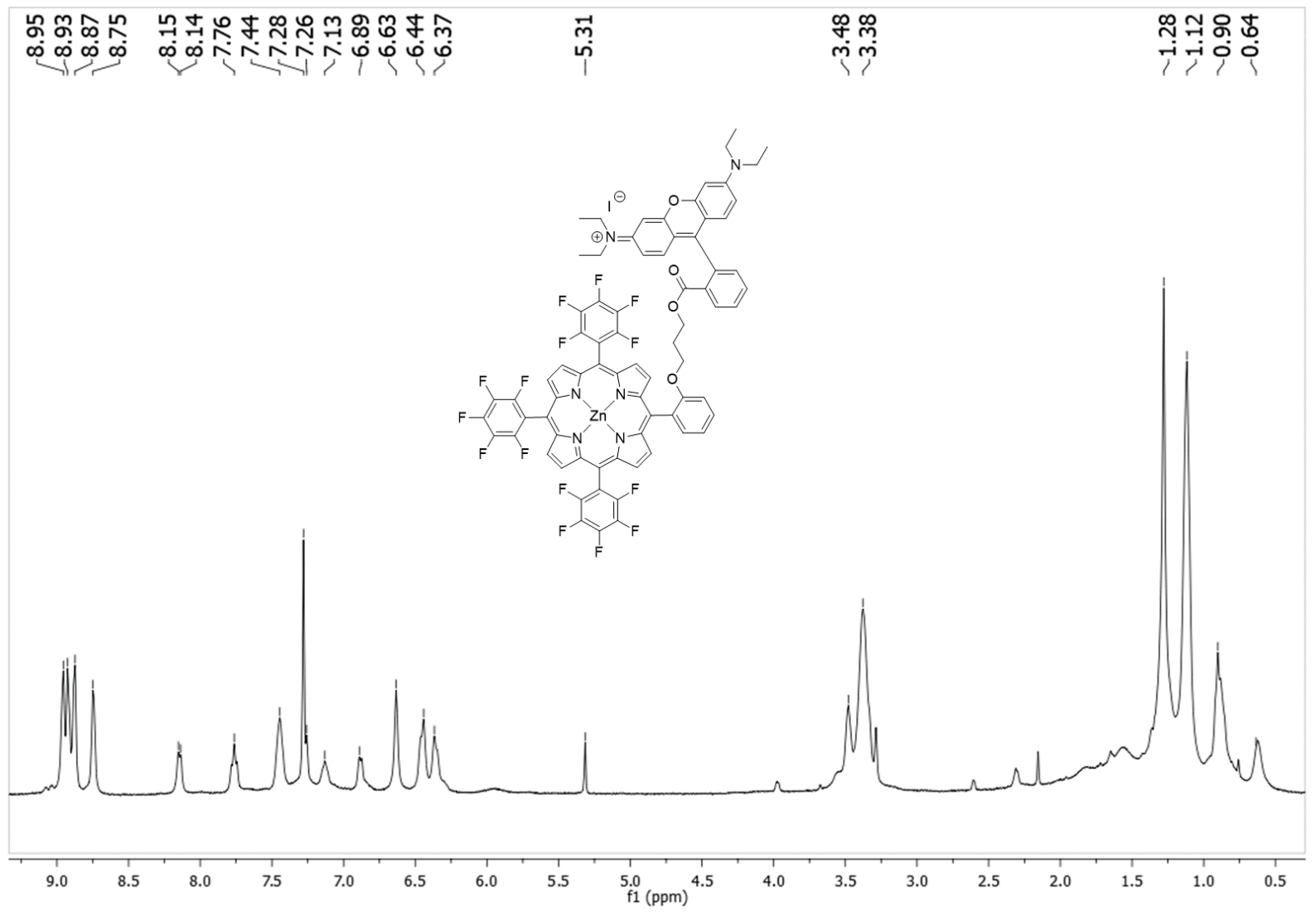


Figure 11. ${ }^{13} \mathrm{C}$ NMR spectrum $\left(100 \mathrm{MHz}, \mathrm{CDCl}_{3}, 298 \mathrm{~K}\right)$ of porphyrin $\mathrm{Zn}(\mathbf{2})$

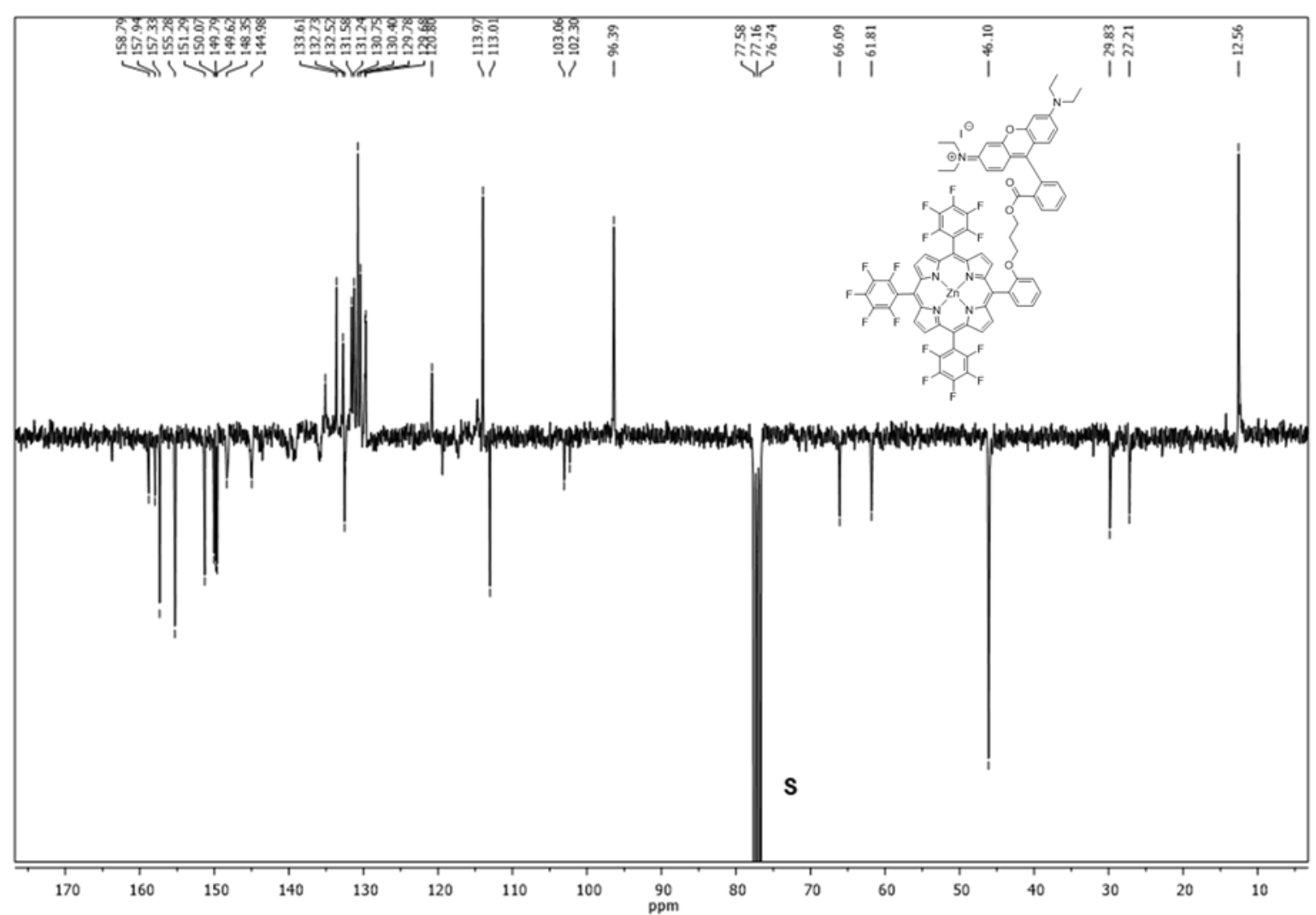

Figure 12. ${ }^{19} \mathrm{~F}$ NMR spectrum $\left(376 \mathrm{MHz}, \mathrm{CDCl}_{3}, 298 \mathrm{~K}\right.$ ) of porphyrin $\mathrm{Zn}(\mathbf{2})$

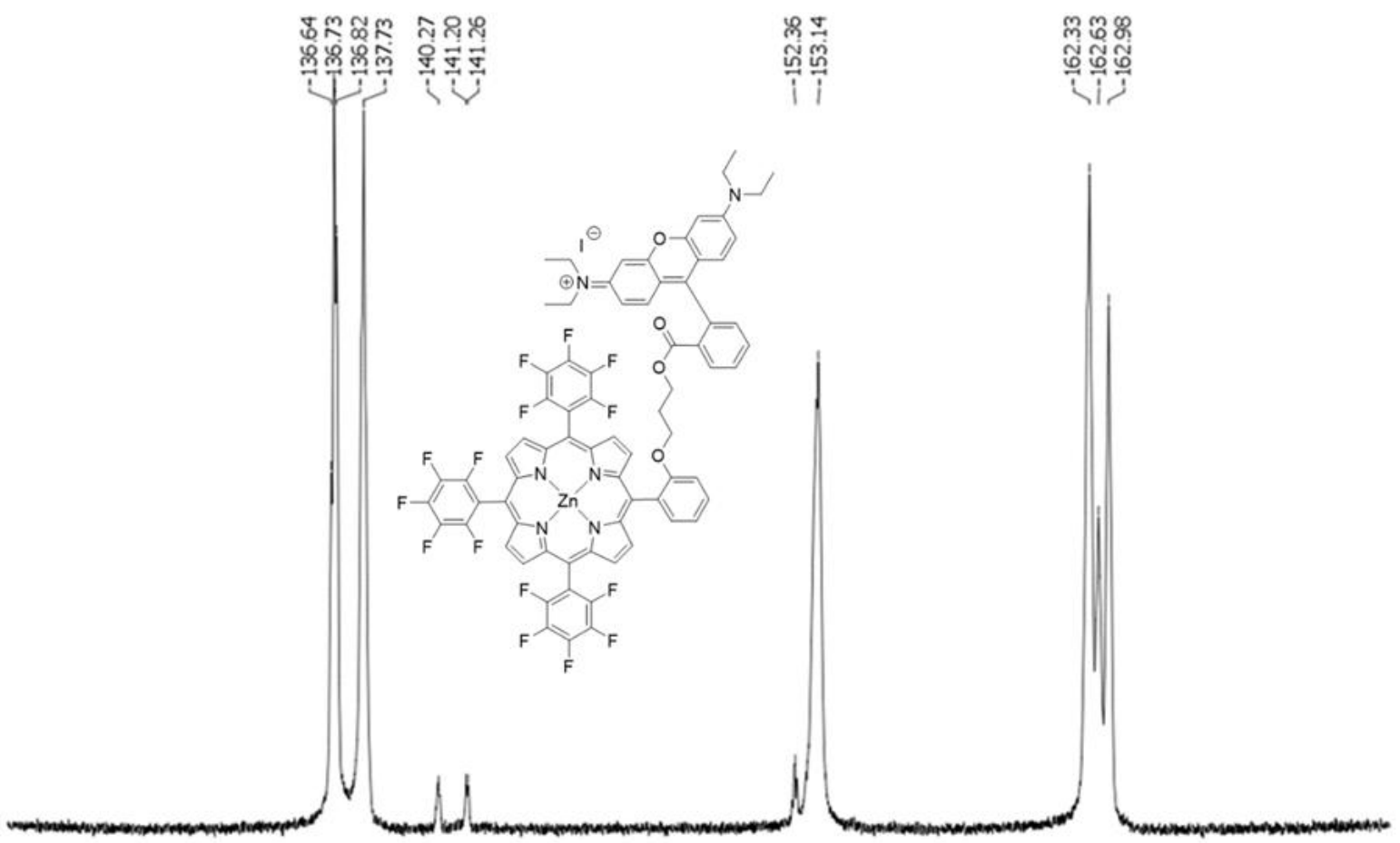

$\begin{array}{lllllllllllllllllllllllllllllllllll}-126 & -128 & -130 & -132 & -134 & -136 & -138 & -140 & -142 & -144 & -146 & -148 & -150 & -152 & -154 & -156 & -158 & -160 & -162 & -164 & -166 & -168 & -170 & -172\end{array}$ 


\section{UV-Vis spectra}

Figure 13. UV-vis spectra of $\mathbf{1}, \mathbf{2}, \mathbf{3}, \mathrm{Zn}(\mathbf{2})$ and Rhodamine $\mathrm{B}$ in $\mathrm{MeOH}$.
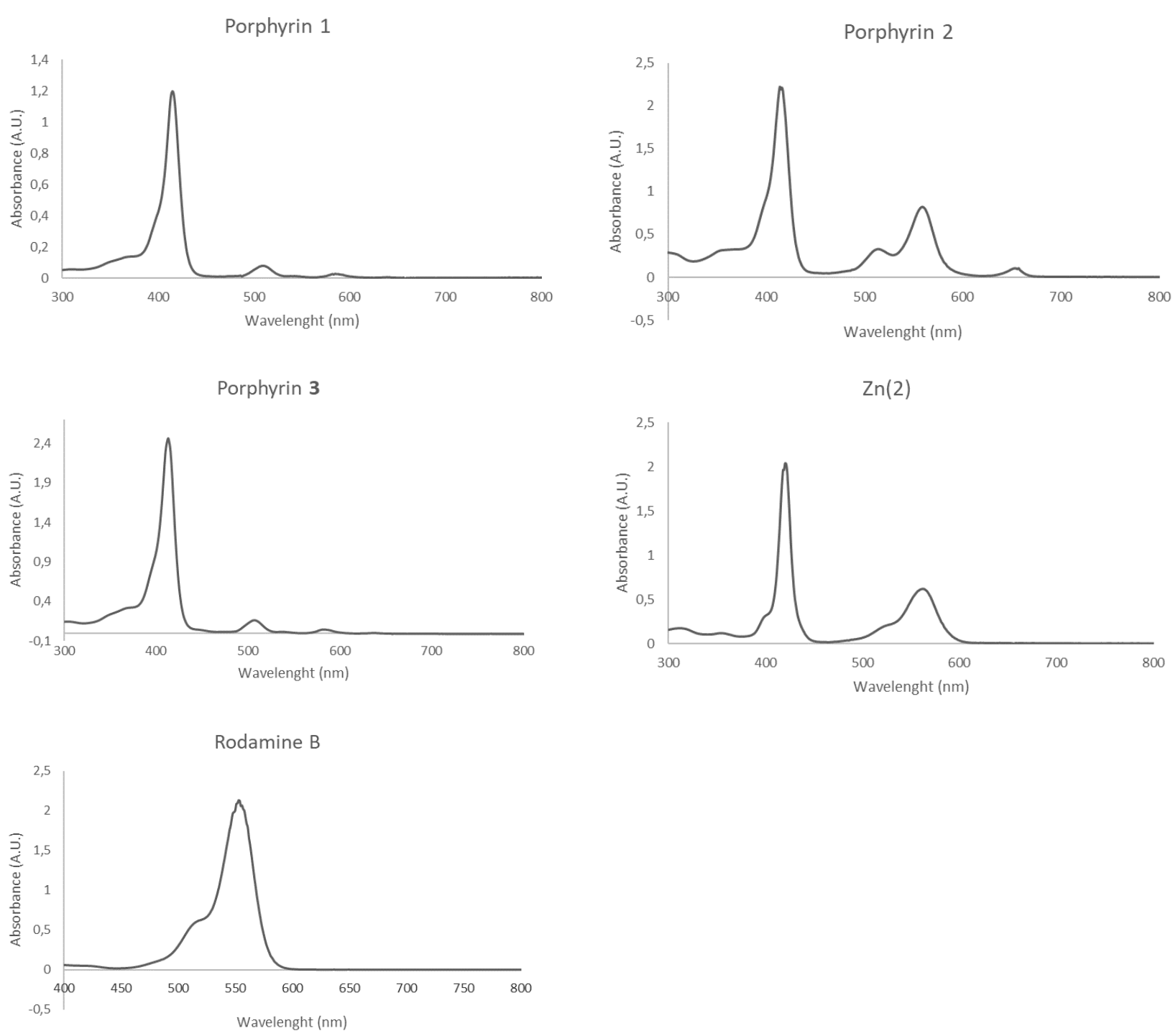


\section{UV-Vis binding tests}

Binding constant measurements were carried out on a Cary 100 Spectrophotometer at $298.0( \pm 0.5){ }^{\circ} \mathrm{C}$ by adding consecutive aliquots of a (DCF)Na solution (1.00 mM) to $2.0 \mathrm{~mL}$ of $1: 1 \mathrm{MeOH} / \mathrm{H}_{2} \mathrm{O}$ (v:v) receptor (2 or $\mathrm{Zn}(2))$ solution $(10.0 \mu \mathrm{M})$. The corresponding spectra was acquired after every (DCF)Na addition. The (DCF)Na solution was prepared by dissolving the required amount of salt into $10.0 \mathrm{~mL}$ of $10.0 \mu \mathrm{M}$ porphyrin solution, in order to keep the concentration of the receptor to a constant value throughout the titration. The absorbance values at $419 \mathrm{~nm}$ were plotted against (DCF)Na concentration and acquired data fitted to Equation $1^{8}$ for a 1:1 molecular complex formation by using a non-linear regression fit program (KaleidaGraph ( ${ }^{8} 4.1$ Synergy Software). Experiments were performed in duplicate and obtained results showed a very good reproducibility. The same procedure was followed in the presence of sodium phenylacetate, sodium salicylate, L tyrosine sodium salt, sodium sarcosinate, L-alanine sodium salt as well as for Fluorescence and Resonance Light Scattering (RLS) studies.

\section{Equation 1}

$\frac{A_{0}-A}{A-A_{\infty}}=\frac{[S]_{t}+[L]_{a}+1 / K_{\text {binding }}-\sqrt{\left([S]_{t}+[L]_{a}+1 / K_{\text {binding }}\right)^{2}-4[S]_{t}[L]_{a}}}{2[S]_{t}}$

Figure 14. UV-vis binding test with porphyrin 2

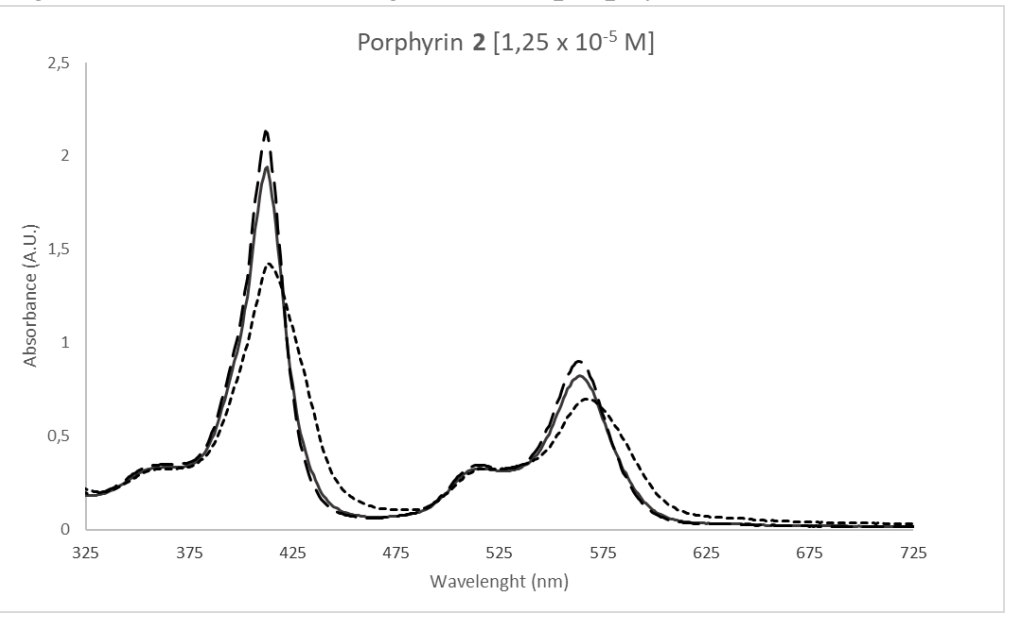

Figure 15. UV-vis binding test with porphyrin $\mathrm{Zn}(\mathbf{2})$

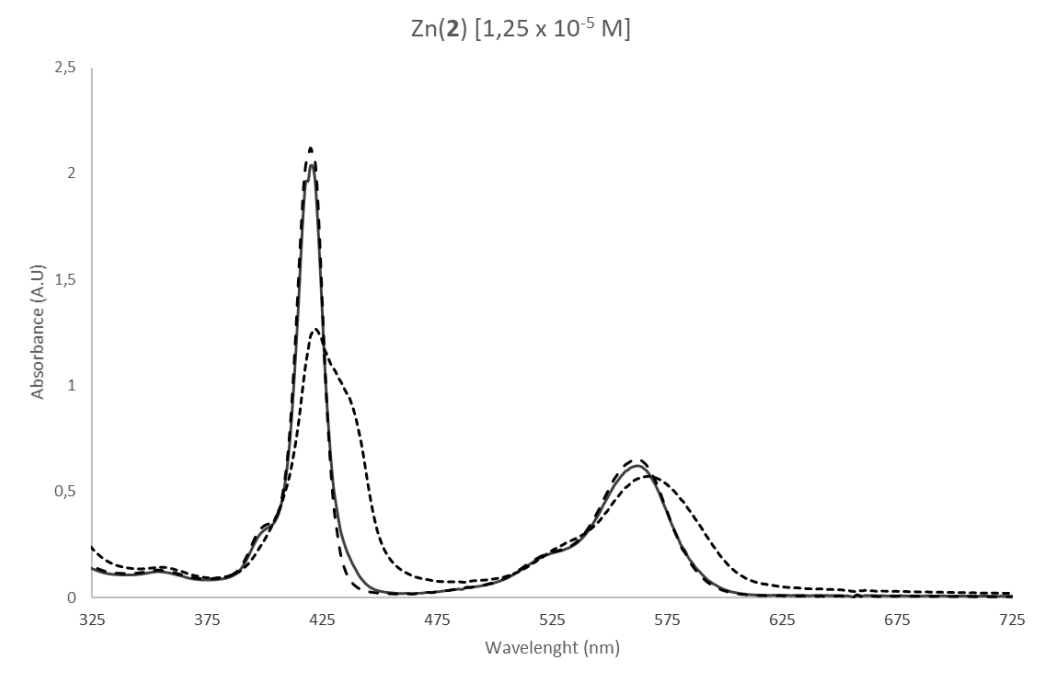


Figure 16. UV-Vis spectral pattern variation of $2\left(10 \mu \mathrm{M} ; \mathrm{MeOH} / \mathrm{H}_{2} \mathrm{O} 1: 1 \mathrm{v}: \mathrm{v}\right)$ upon addition of (DCF)Na and equimolar amount of sodium salicylate ( $\mathrm{SalONa})$.

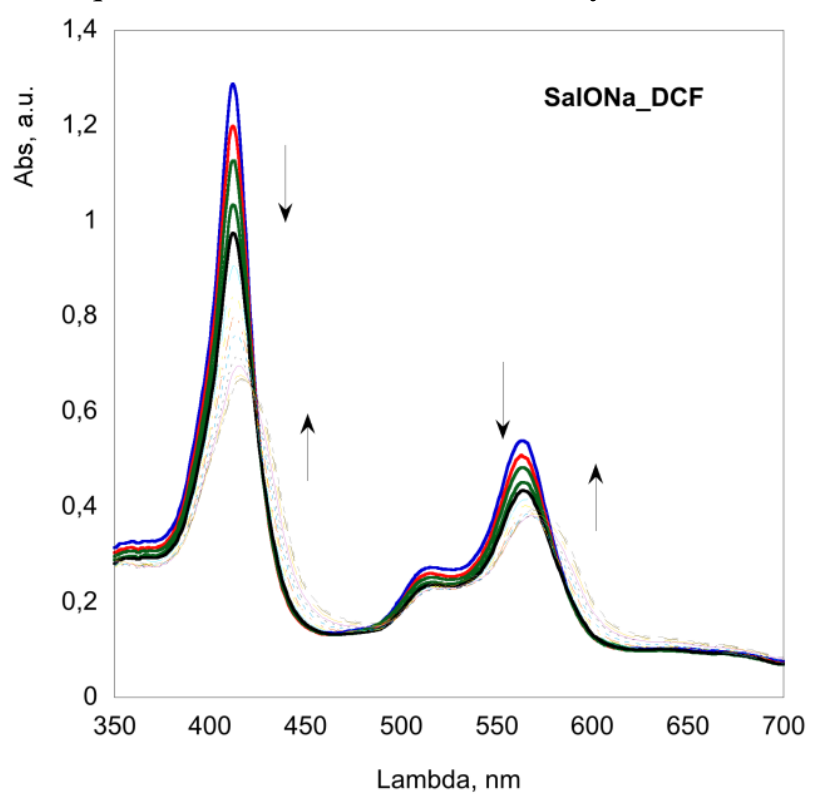

Figure 17. Non-linear regression fit, and calculated binding constant value (Langmuir type equation, see above) for the 2@DCF formation in the presence of equimolar amount of sodium salicylate.

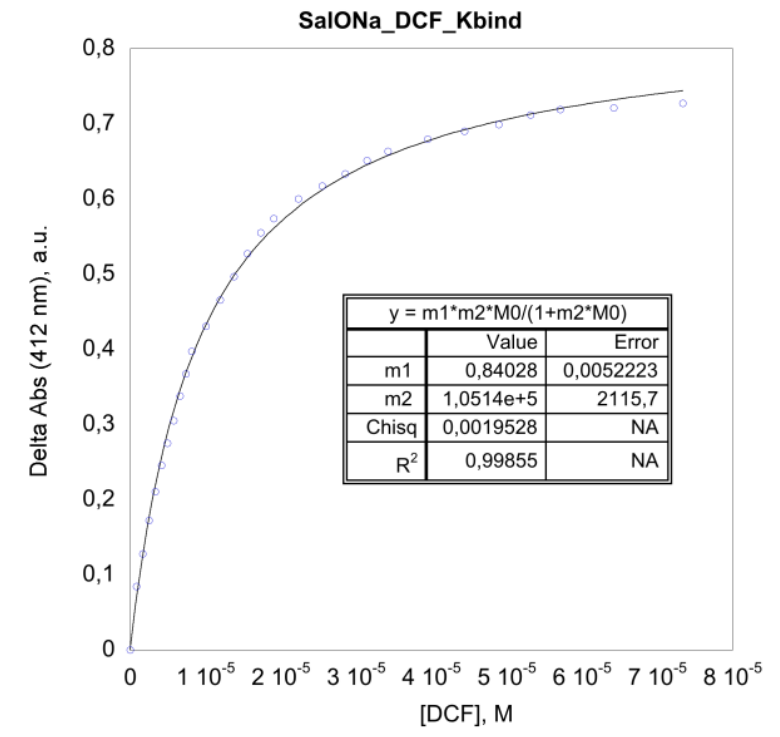




\section{Fluorescence experiments}

Figure 18. Fluorescence emission spectrum of 2 at $\lambda_{\mathrm{exc}}=412 \mathrm{~nm}$.

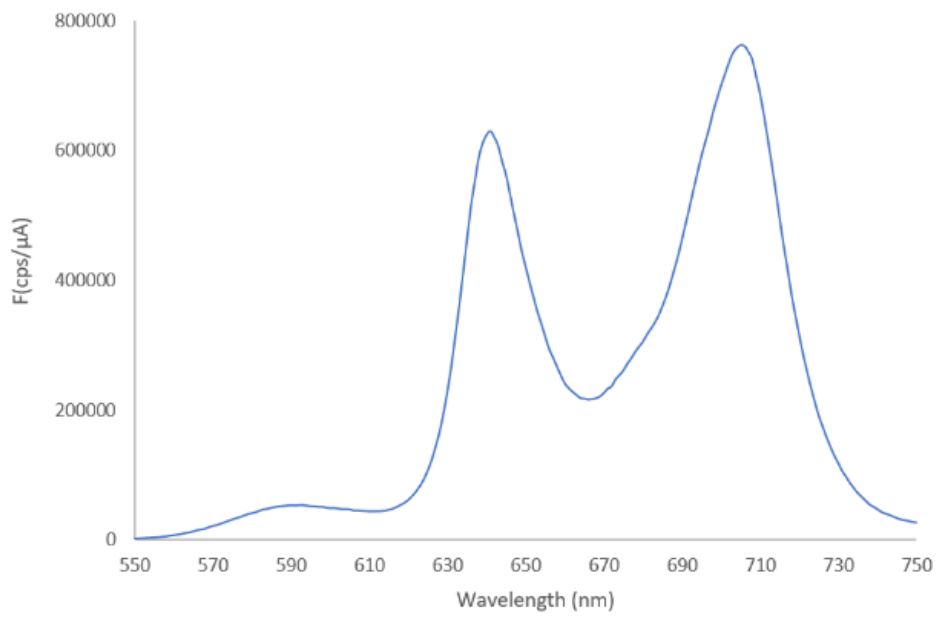

Figure 19. Fluorescence excitation spectrum of 2 at $\lambda_{\mathrm{em}}=590 \mathrm{~nm}$ (Rhodamine emission)

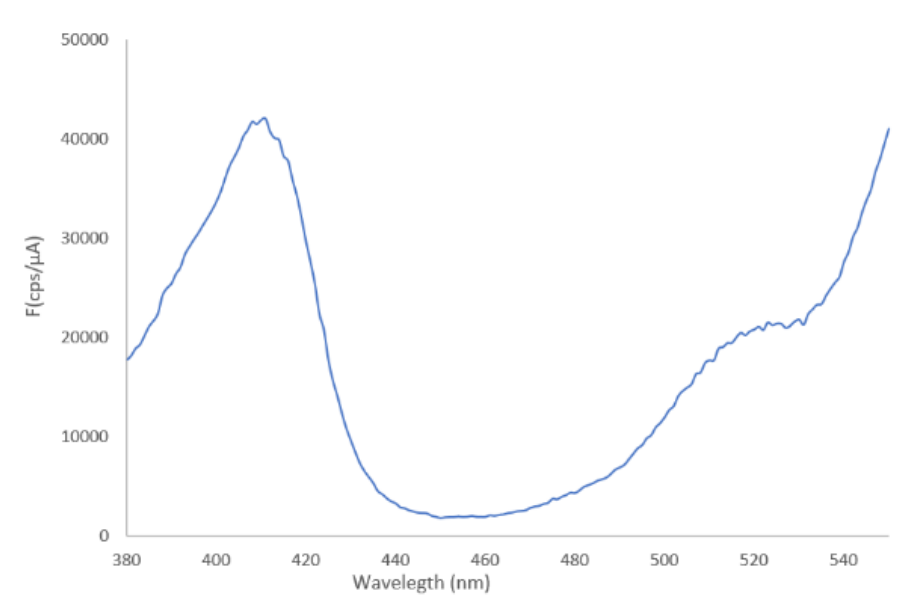

Figure 20. Fluorescence excitation spectrum of 2 at $\lambda_{\mathrm{em}}=705 \mathrm{~nm}$ (Porphyrin emission)

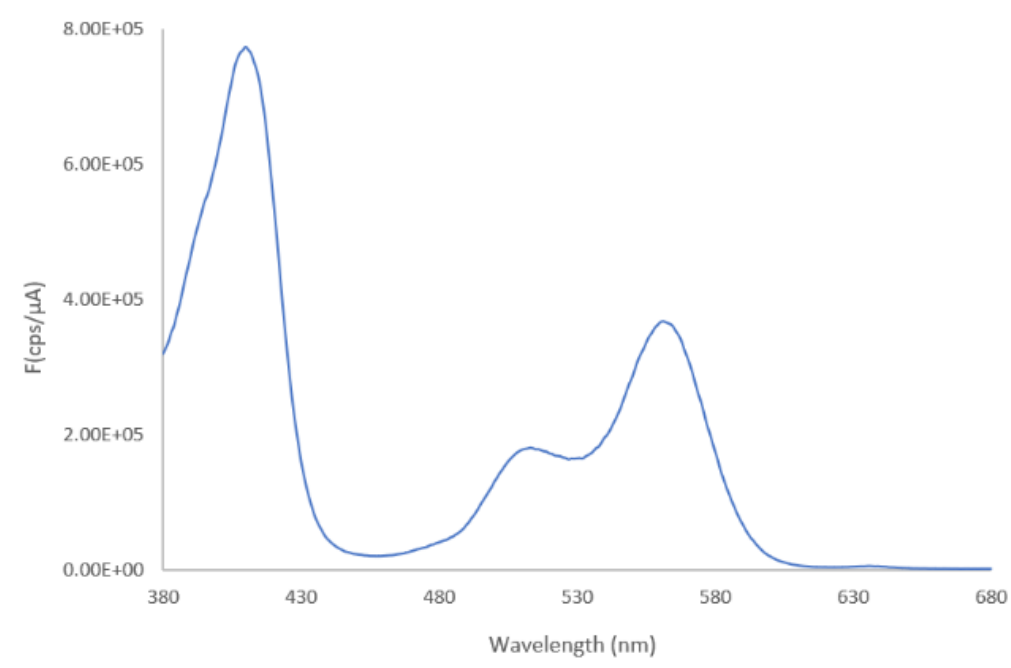


Figure 21. Fluorescence emission spectra of 2 for increasing concentrations of DCF. A: $\lambda_{\mathrm{ex}}=412 \mathrm{~nm}$ (Porphyrin absorption); B: $\lambda_{\mathrm{ex}}=555 \mathrm{~nm}$ (Rhodamine preferential absorption).
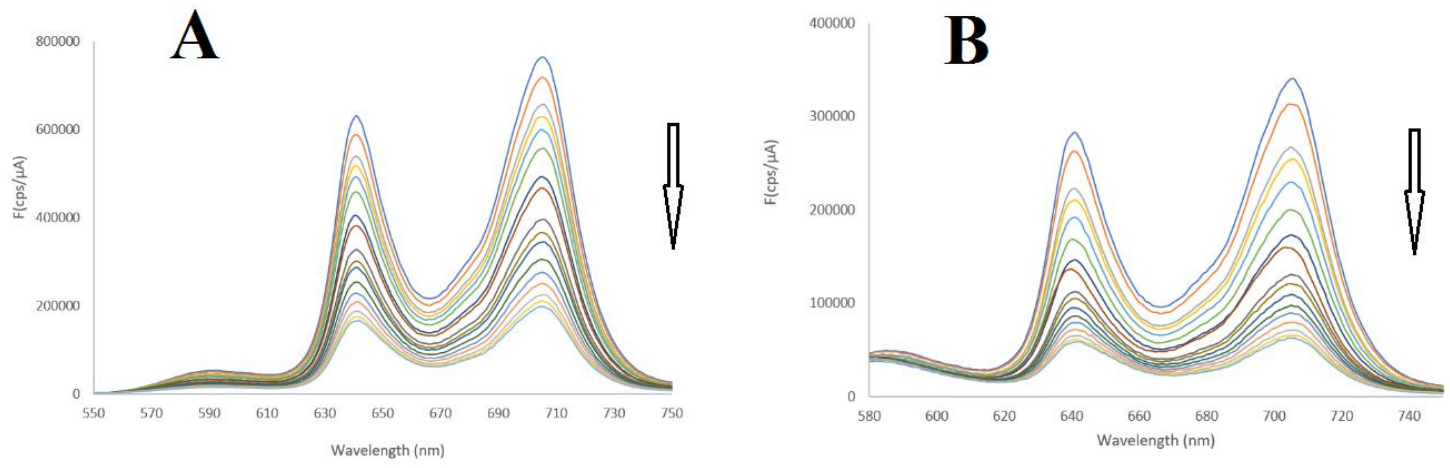

Figure 22. 2/DCF binding isotherm from fluorescence intensities of 2 for different (DCF)Na concentrations. The analogous of Equation 1 was applied.

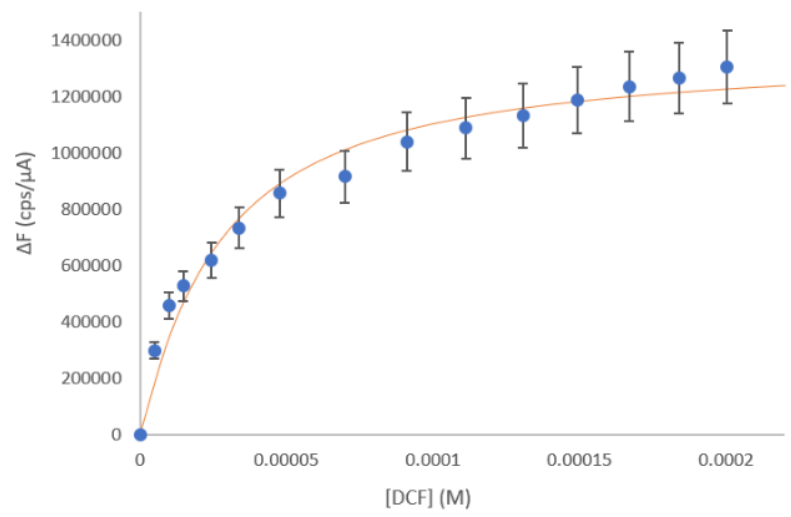

Figure 23. Fluorescence time decays of 2 for different 2/DCF concentration ratios $\left(\lambda_{\mathrm{ex}}=342 \mathrm{~nm} ; \lambda_{\mathrm{em}}=645\right.$ $\mathrm{nm})$.

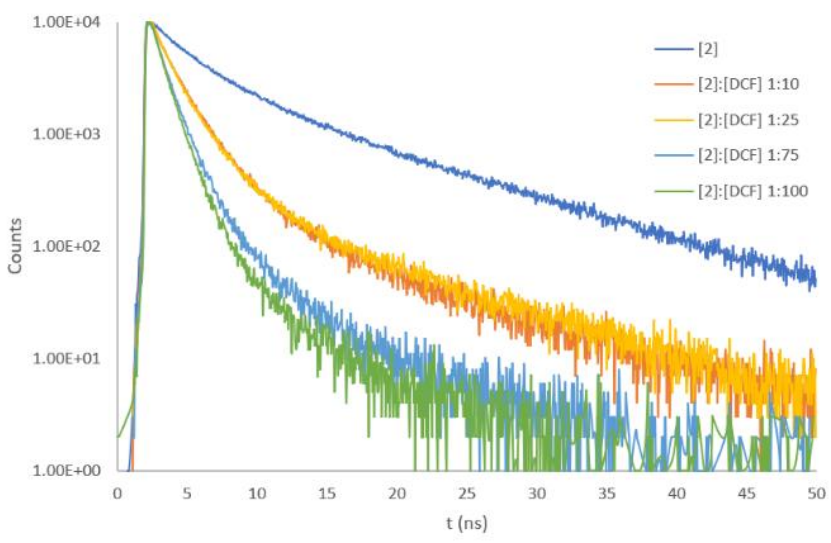

Table 1. Time decay parameters of $\mathbf{2}$ (porphyrin emission) for different $\mathbf{2} / \mathrm{DCF}$ concentration ratios $\left(\lambda_{\mathrm{ex}}=342\right.$ $\mathrm{nm} ; \lambda_{\mathrm{em}}=645 \mathrm{~nm}$ ).

\begin{tabular}{lcccccccc}
\hline \multicolumn{1}{c}{$[2]:[\mathrm{DCF}]$} & $\tau_{1}(\mathrm{~ns})$ & $\alpha_{1}$ & $\tau_{2}(\mathrm{~ns})$ & $\alpha_{2}$ & $\tau_{3}(\mathrm{~ns})$ & $\alpha_{3}$ & $\langle\tau>(\mathrm{ns})$ & $\chi^{2}$ \\
\hline $1: 0$ & 11.6 & 0.38 & 3.45 & 0.46 & 1.0 & 0.17 & 6.3 & 1.303 \\
$1: 10$ & 10.2 & 0.02 & 2.2 & 0.52 & 0.95 & 0.46 & 1.8 & 1.154 \\
$1: 25$ & 10.0 & 0.03 & 2.1 & 0.48 & 0.88 & 0.49 & 1.7 & 1.031 \\
$1: 75$ & 6.1 & 0.01 & 1.5 & 0.41 & 0.72 & 0.58 & 1.1 & 1.052 \\
$1: 100$ & 5.0 & 0.01 & 1.2 & 0.50 & 0.64 & 0.49 & 1.0 & 0.913 \\
\hline
\end{tabular}




\section{X-ray single-crystal structure determination.}

Crystal data for 3 are reported in Table 2. All hydrogen atoms of porphyrin molecule, except the $\mathrm{H}$ atoms of methyl groups, were located from the difference Fourier map and refined freely with isotropic displacement parameters. Methyl hydrogens and $\mathrm{H}$-atoms of the guest molecule ( $n$-hexane) were placed in geometrically calculated positions and included in the refinement using a riding model in conjunction with a $U_{\text {iso }}(\mathrm{H})=1.2$ $U_{\text {eq }}\left(\mathrm{CH}_{2}, \mathrm{CH}\right)$ or $1.5 U_{\text {eq }}\left(\mathrm{CH}_{3}\right)$ constraint. All non-hydrogen atoms were refined with full occupancy and anisotropic displacement parameters except for hexane carbon atoms. ISOR instruction was used for F2 atom as otherwise it went non-positive definite. The solvate hexane molecule was find to be disordered and refined isotropically over two positions using a suitable model $(49.7,50.1 \%)$. Geometry constraints (SADI) were used to keep reasonable bond distances.

Table 2. Crystal data and structure refinement for $\mathbf{3}$.

Chemical formula

Empirical formula

Formula weight $\left(\mathrm{g} \cdot \mathrm{mol}^{-1}\right)$

Temperature $(\mathrm{K})$

Wavelength $(\AA)$

Crystal system

Space group

a / $\AA$

$\mathrm{b} / \AA$

$\mathrm{c} / \AA$

$\alpha(\operatorname{deg})$

$\beta$ (deg)

$\gamma(\operatorname{deg})$

$\mathrm{V} / \AA^{3}$

$\mathrm{Z}$

$D_{\text {calc. }}\left(\mathrm{g} \cdot \mathrm{cm}^{-3}\right)$

$\mu\left(\mathrm{mm}^{-1}\right)$

$\theta$ range for data collection (deg)

Index ranges

Reflections collected

Independent reflections

Completeness to theta

Data/restraints/parameters

$F(000)$

Goodness-of-fit on $\mathrm{F}^{2}$

Final $R$ indices [I $>2 \operatorname{sigma}(\mathrm{I})]$

$R$ indices (all data)

$\Delta \rho \max , \min \left(\mathrm{e} \cdot \AA^{-3}\right)$
$\left[\mathrm{C}_{53} \mathrm{H}_{35} \mathrm{~F}_{15} \mathrm{~N}_{5} \mathrm{O}\right] \mathrm{I} \cdot 0.5\left(\mathrm{C}_{6} \mathrm{H}_{14}\right)$

$\mathrm{C}_{56} \mathrm{H}_{42} \mathrm{~F}_{15} \mathrm{I} \mathrm{N}_{5} \mathrm{O}$

1212.85

$150(2)$

0.71073

Triclinic

$P-1$

$11.465(3)$

$15.353(4)$

$16.170(5)$

$103.382(3)$

90.662(3)

106.684(3)

$14687(2)$

12

1.524

0.704

1.3 to 25.0

$-13<=\mathrm{h}<=13,-18<=\mathrm{k}<=18,-19<=1<=19$

23475

$9334[\mathrm{R}($ int $)=0.0383]$

$99.9 \%$

9334 / 21 / 775

1218

1.039

$R_{1}=0.0580, \mathrm{w} R_{2}=0.1461$

$R_{1}=0.0855, \mathrm{w} R_{2}=0.1719$

2.432 and -0.775 
Table 3. Selected bond lengths $[\AA]$ and angles $\left[{ }^{\circ}\right]$ for 3 .

\begin{tabular}{|c|c|c|c|}
\hline $\mathrm{N}(1)-\mathrm{C}(1)$ & $1.365(6)$ & $\mathrm{N}(1)-\mathrm{C}(4)-\mathrm{C}(5)$ & $125.7(5)$ \\
\hline $\mathrm{N}(1)-\mathrm{C}(4)$ & $1.365(7)$ & $\mathrm{N}(1)-\mathrm{C}(4)-\mathrm{C}(3)$ & $109.6(5)$ \\
\hline $\mathrm{N}(2)-\mathrm{C}(6)$ & $1.362(7)$ & $\mathrm{C}(5)-\mathrm{C}(4)-\mathrm{C}(3)$ & $124.6(5)$ \\
\hline $\mathrm{N}(2)-\mathrm{C}(9)$ & $1.363(7)$ & $\mathrm{C}(4)-\mathrm{C}(5)-\mathrm{C}(6)$ & $126.8(5)$ \\
\hline $\mathrm{N}(3)-\mathrm{C}(14)$ & $1.364(6)$ & $\mathrm{C}(4)-\mathrm{C}(5)-\mathrm{C}(21)$ & $117.4(5)$ \\
\hline $\mathrm{N}(3)-\mathrm{C}(11)$ & $1.370(6)$ & $C(6)-C(5)-C(21)$ & $115.6(5)$ \\
\hline $\mathrm{N}(4)-\mathrm{C}(16)$ & $1.364(7)$ & $\mathrm{N}(2)-\mathrm{C}(6)-\mathrm{C}(5)$ & $125.5(5)$ \\
\hline $\mathrm{N}(4)-\mathrm{C}(19)$ & $1.372(6)$ & $\mathrm{N}(2)-\mathrm{C}(6)-\mathrm{C}(7)$ & $107.5(5)$ \\
\hline $\mathrm{C}(1)-\mathrm{C}(20)$ & $1.401(7)$ & $C(5)-C(6)-C(7)$ & $126.9(5)$ \\
\hline$C(1)-C(2)$ & $1.443(8)$ & $\mathrm{C}(8)-\mathrm{C}(7)-\mathrm{C}(6)$ & $108.0(5)$ \\
\hline$C(2)-C(3)$ & $1.330(8)$ & $\mathrm{C}(7)-\mathrm{C}(8)-\mathrm{C}(9)$ & $107.8(5)$ \\
\hline$C(3)-C(4)$ & $1.445(8)$ & $\mathrm{N}(2)-\mathrm{C}(9)-\mathrm{C}(10)$ & $126.6(4)$ \\
\hline $\mathrm{C}(4)-\mathrm{C}(5)$ & $1.383(8)$ & $\mathrm{N}(2)-\mathrm{C}(9)-\mathrm{C}(8)$ & $107.2(5)$ \\
\hline $\mathrm{C}(5)-\mathrm{C}(6)$ & $1.396(8)$ & $\mathrm{C}(10)-\mathrm{C}(9)-\mathrm{C}(8)$ & $126.2(5)$ \\
\hline$C(5)-C(21)$ & $1.504(7)$ & $\mathrm{C}(9)-\mathrm{C}(10)-\mathrm{C}(11)$ & $124.7(5)$ \\
\hline$C(6)-C(7)$ & $1.421(8)$ & $\mathrm{C}(9)-\mathrm{C}(10)-\mathrm{C}(27)$ & $117.5(4)$ \\
\hline $\mathrm{C}(7)-\mathrm{C}(8)$ & $1.349(8)$ & $\mathrm{C}(11)-\mathrm{C}(10)-\mathrm{C}(27)$ & $117.5(5)$ \\
\hline $\mathrm{C}(8)-\mathrm{C}(9)$ & $1.428(7)$ & $\mathrm{N}(3)-\mathrm{C}(11)-\mathrm{C}(10)$ & $126.0(5)$ \\
\hline$C(9)-C(10)$ & $1.396(7)$ & $\mathrm{N}(3)-\mathrm{C}(11)-\mathrm{C}(12)$ & $109.8(4)$ \\
\hline $\mathrm{C}(10)-\mathrm{C}(11)$ & $1.398(7)$ & $\mathrm{C}(10)-\mathrm{C}(11)-\mathrm{C}(12)$ & $124.1(5)$ \\
\hline $\mathrm{C}(10)-\mathrm{C}(27)$ & $1.489(7)$ & $\mathrm{C}(13)-\mathrm{C}(12)-\mathrm{C}(11)$ & $107.3(5)$ \\
\hline $\mathrm{C}(11)-\mathrm{C}(12)$ & $1.442(8)$ & $\mathrm{C}(12)-\mathrm{C}(13)-\mathrm{C}(14)$ & $106.7(5)$ \\
\hline $\mathrm{C}(12)-\mathrm{C}(13)$ & $1.340(8)$ & $\mathrm{N}(3)-\mathrm{C}(14)-\mathrm{C}(15)$ & $125.2(5)$ \\
\hline $\mathrm{C}(13)-\mathrm{C}(14)$ & $1.446(7)$ & $\mathrm{N}(3)-\mathrm{C}(14)-\mathrm{C}(13)$ & $110.1(4)$ \\
\hline $\mathrm{C}(14)-\mathrm{C}(15)$ & $1.398(7)$ & $\mathrm{C}(15)-\mathrm{C}(14)-\mathrm{C}(13)$ & $124.6(5)$ \\
\hline$C(15)-C(16)$ & $1.392(7)$ & $\mathrm{C}(16)-\mathrm{C}(15)-\mathrm{C}(14)$ & $126.9(5)$ \\
\hline $\mathrm{C}(15)-\mathrm{C}(33)$ & $1.499(7)$ & $\mathrm{C}(16)-\mathrm{C}(15)-\mathrm{C}(33)$ & $116.5(4)$ \\
\hline $\mathrm{C}(16)-\mathrm{C}(17)$ & $1.430(7)$ & $\mathrm{C}(14)-\mathrm{C}(15)-\mathrm{C}(33)$ & $116.6(5)$ \\
\hline $\mathrm{C}(17)-\mathrm{C}(18)$ & $1.347(8)$ & $N(4)-C(16)-C(15)$ & $125.3(5)$ \\
\hline $\mathrm{C}(18)-\mathrm{C}(19)$ & $1.433(7)$ & $N(4)-C(16)-C(17)$ & $108.0(4)$ \\
\hline$C(19)-C(20)$ & $1.391(7)$ & $\mathrm{C}(15)-\mathrm{C}(16)-\mathrm{C}(17)$ & $126.7(5)$ \\
\hline $\mathrm{C}(20)-\mathrm{C}(39)$ & $1.497(7)$ & $\mathrm{C}(18)-\mathrm{C}(17)-\mathrm{C}(16)$ & $107.6(5)$ \\
\hline $\mathrm{C}(32)-\mathrm{O}(1)$ & $1.377(7)$ & $\mathrm{C}(17)-\mathrm{C}(18)-\mathrm{C}(19)$ & $108.2(5)$ \\
\hline $\mathrm{N}(1 \mathrm{~S})-\mathrm{C}(3 \mathrm{~B})$ & $1.505(8)$ & $\mathrm{N}(4)-\mathrm{C}(19)-\mathrm{C}(20)$ & $125.8(4)$ \\
\hline $\mathrm{N}(1 \mathrm{~S})-\mathrm{C}(4 \mathrm{~B})$ & $1.507(8)$ & $\mathrm{N}(4)-\mathrm{C}(19)-\mathrm{C}(18)$ & $107.3(4)$ \\
\hline $\mathrm{N}(1 \mathrm{~S})-\mathrm{C}(8 \mathrm{~B})$ & $1.508(7)$ & $\mathrm{C}(20)-\mathrm{C}(19)-\mathrm{C}(18)$ & $126.9(5)$ \\
\hline $\mathrm{N}(1 \mathrm{~S})-\mathrm{C}(6 \mathrm{~B})$ & $1.522(7)$ & $\mathrm{C}(19)-\mathrm{C}(20)-\mathrm{C}(1)$ & $126.1(5)$ \\
\hline $\mathrm{O}(1)-\mathrm{C}(1 \mathrm{~B})$ & $1.425(9)$ & $\mathrm{C}(19)-\mathrm{C}(20)-\mathrm{C}(39)$ & $116.8(4)$ \\
\hline $\mathrm{C}(1 \mathrm{~B})-\mathrm{C}(2 \mathrm{~B})$ & $1.521(9)$ & $\mathrm{C}(1)-\mathrm{C}(20)-\mathrm{C}(39)$ & $117.1(5)$ \\
\hline $\mathrm{C}(2 \mathrm{~B})-\mathrm{C}(3 \mathrm{~B})$ & $1.522(9)$ & $\mathrm{O}(1)-\mathrm{C}(32)-\mathrm{C}(31)$ & $119.7(5)$ \\
\hline $\mathrm{C}(4 \mathrm{~B})-\mathrm{C}(5 \mathrm{~B})$ & $1.521(9)$ & $\mathrm{O}(1)-\mathrm{C}(32)-\mathrm{C}(27)$ & $119.6(5)$ \\
\hline $\mathrm{C}(6 \mathrm{~B})-\mathrm{C}(7 \mathrm{~B})$ & $1.505(10)$ & $\mathrm{C}(3 \mathrm{~B})-\mathrm{N}(1 \mathrm{~S})-\mathrm{C}(4 \mathrm{~B})$ & $111.5(5)$ \\
\hline \multirow[t]{3}{*}{$\mathrm{C}(8 \mathrm{~B})-\mathrm{C}(9 \mathrm{~B})$} & $1.525(9)$ & $\mathrm{C}(3 \mathrm{~B})-\mathrm{N}(1 \mathrm{~S})-\mathrm{C}(8 \mathrm{~B})$ & $106.3(4)$ \\
\hline & & $\mathrm{C}(4 \mathrm{~B})-\mathrm{N}(1 \mathrm{~S})-\mathrm{C}(8 \mathrm{~B})$ & $111.6(5)$ \\
\hline & & $\mathrm{C}(3 \mathrm{~B})-\mathrm{N}(1 \mathrm{~S})-\mathrm{C}(6 \mathrm{~B})$ & $110.3(5)$ \\
\hline $\mathrm{C}(1)-\mathrm{N}(1)-\mathrm{C}(4)$ & $105.8(4)$ & $\mathrm{C}(4 \mathrm{~B})-\mathrm{N}(1 \mathrm{~S})-\mathrm{C}(6 \mathrm{~B})$ & $106.3(4)$ \\
\hline $\mathrm{C}(6)-\mathrm{N}(2)-\mathrm{C}(9)$ & $109.4(4)$ & $\mathrm{C}(8 \mathrm{~B})-\mathrm{N}(1 \mathrm{~S})-\mathrm{C}(6 \mathrm{~B})$ & $110.8(5)$ \\
\hline $\mathrm{C}(14)-\mathrm{N}(3)-\mathrm{C}(11)$ & $106.0(4)$ & $\mathrm{C}(32)-\mathrm{O}(1)-\mathrm{C}(1 \mathrm{~B})$ & $116.0(5)$ \\
\hline $\mathrm{C}(16)-\mathrm{N}(4)-\mathrm{C}(19)$ & $108.9(4)$ & $\mathrm{O}(1)-\mathrm{C}(1 \mathrm{~B})-\mathrm{C}(2 \mathrm{~B})$ & $106.9(6)$ \\
\hline $\mathrm{N}(1)-\mathrm{C}(1)-\mathrm{C}(20)$ & $125.2(5)$ & $\mathrm{C}(1 \mathrm{~B})-\mathrm{C}(2 \mathrm{~B})-\mathrm{C}(3 \mathrm{~B})$ & $109.0(5)$ \\
\hline $\mathrm{N}(1)-\mathrm{C}(1)-\mathrm{C}(2)$ & $110.5(4)$ & $\mathrm{N}(1 \mathrm{~S})-\mathrm{C}(3 \mathrm{~B})-\mathrm{C}(2 \mathrm{~B})$ & $115.3(5)$ \\
\hline$C(20)-C(1)-C(2)$ & $124.3(5)$ & $\mathrm{N}(1 \mathrm{~S})-\mathrm{C}(4 \mathrm{~B})-\mathrm{C}(5 \mathrm{~B})$ & $114.5(5)$ \\
\hline$C(3)-C(2)-C(1)$ & $106.5(5)$ & $\mathrm{C}(7 \mathrm{~B})-\mathrm{C}(6 \mathrm{~B})-\mathrm{N}(1 \mathrm{~S})$ & $114.2(5)$ \\
\hline $\mathrm{C}(2)-\mathrm{C}(3)-\mathrm{C}(4)$ & $107.6(5)$ & $\mathrm{N}(1 \mathrm{~S})-\mathrm{C}(8 \mathrm{~B})-\mathrm{C}(9 \mathrm{~B})$ & $115.3(5)$ \\
\hline
\end{tabular}


Table 4. Hydrogen-bond geometry for $\mathbf{3}\left(\AA{ }^{\circ}{ }^{\circ}\right)$

\begin{tabular}{lllll}
\hline Donor- H$\cdots$ Acceptor & $D-\mathrm{H}$ & $\mathrm{H} \cdots \mathrm{A}$ & $D \cdots \mathrm{A}$ & $D-\mathrm{H} \cdots \mathrm{A}$ \\
\hline $\mathrm{N} 2-\mathrm{H} 2 \mathrm{~N} \cdots \mathrm{N} 1$ & $0.70(9)$ & $2.48(9)$ & $2.913(7)$ & $123(9)$ \\
$\mathrm{N} 2-\mathrm{H} 2 \mathrm{~N} \cdots \mathrm{N} 3$ & $0.70(9)$ & $2.43(9)$ & $2.897(7)$ & $126(8)$ \\
$\mathrm{N} 4-\mathrm{H} 4 \mathrm{~N} \cdots \mathrm{N} 1$ & $0.75(9)$ & $2.49(9)$ & $2.895(7)$ & $116(8)$ \\
$\mathrm{N} 4-\mathrm{H} 4 \mathrm{~N} \cdots \mathrm{N} 3$ & $0.75(9)$ & $2.36(8)$ & $2.905(6)$ & $131(8)$ \\
\hline
\end{tabular}

\section{Conformational analysis.}

The porphyrin 3 adopts a "close" conformation in which the amino-alkyl side arm is folded toward the core of the macrocycle, with the terminal methyl groups of the pendant triethylammonium moiety pointing toward the pyrrole rings (I, II, IV). The lateral displacement of the side arm from the porphyrin centroid is generated by a gauche/anti/anti/anti(N)/anti conformation sequence along the main chain.

The porphyrin core is quasi-planar with an average deviation of the macrocycle atoms from their least squares plane $(\Delta 24$, Table 5$)$ of only $0.065 \AA$. The larger deviations are associated with the meso-carbon $\left(\mathrm{C}_{m}\right)$ $\mathrm{C} 10(0.106(5) \AA)$ bonded to the para substituted aryl group and with the $\mathrm{C}_{\beta}$ of the neighbouring pyrrole rings (-0.089(6)-0.172(6) $\AA$ ). Likewise, the $\mathrm{C}_{\alpha}-\mathrm{C}_{\mathrm{m}}-\mathrm{C}_{\alpha}$ angle between the $\alpha$-pyrrolic and meso carbons varies only slightly (Table 5) with the smallest value and the bigger deviation at meso-C10 position again $\left(124.7(5)^{\circ}\right)$.

The overall slightly distortion results essentially in a saddle shaped conformation characterized by an alternating displacement of the pyrrole rings N(I), (III) and (IV) below and above the mean plane (Figure 24). ${ }^{9}$ A closer look of the out-of-plane distortion pattern suggests a small contribute from wave conformation involving the pyrrolic unit $\mathrm{N}$ (II) (toward which the side arm of porphyrin is bent). ${ }^{10}$ The minor deformation from planarity of porphyrin macrocycle and the different role of the pyrrolic units is also reflected by the values of the dihedral angle between the pyrrole rings and the $\mathrm{N}_{4}$-core mean plane (Table 5).

All the conformational data seem indicate that the small distortions observed for the macrocycle are essentially due to the presence and orientation of the "long" alkyl chain on the para position of a meso-aryl group. Despite the asymmetric substituent arrangement, the inner cavity shape composed by the four $\mathrm{N}$ atoms is square-like as indicated by the core elongation factor $(\Xi=0.013 \AA)$ and by the distances between the neighbouring $\mathrm{N}-\mathrm{N}$ atoms $(2.895(7)-2.913(7) \AA)$. The $\mathrm{N}-\mathrm{H}$ groups are involved in bifurcated $\mathrm{N}-\mathrm{H} \cdots(\mathrm{N}, \mathrm{N})$ intramolecular hydrogen bonds (Table 4) and result only slightly tilted out of the plane of macrocycle. 
Table 5. Selected conformational parameters for $\mathbf{3}$ (deviations and distances in $\AA$, angles in ${ }^{\circ}$ )

\begin{tabular}{llll}
\hline Core size $\otimes^{a}$ & 2.053 & $\mathrm{C}_{\alpha}-\mathrm{C}_{15}-\mathrm{C}_{\alpha}$ & $126.9(5)$ \\
Core elongation $\Xi^{b}$ & 0.013 & $\mathrm{C}_{\alpha}-\mathrm{C}_{20}-\mathrm{C}_{\alpha}$ & $126.1(5)$ \\
$\Delta 24^{c}$ & 0.065 & cis $\mathrm{C}_{\alpha}-\mathrm{N} \cdots \mathrm{N}-\mathrm{C}_{\alpha}{ }^{g}$ & 4.2 \\
$\mathrm{~N} 1 \cdots \mathrm{N} 2$ & $2.913(7)$ & trans $\mathrm{C}_{\alpha}-\mathrm{N} \cdots \mathrm{N}-\mathrm{C}_{\alpha}$ & 177.1 \\
$\mathrm{~N} 2 \cdots \mathrm{N} 3$ & $2.897(7)$ & $\varphi_{\mathrm{pyr}} \mathrm{N} 1^{h}$ & 1.89 \\
$\mathrm{~N} 3 \cdots \mathrm{N} 4$ & $2.905(6)$ & $\varphi_{\mathrm{pyr}} \mathrm{N} 2$ & 7.24 \\
$\mathrm{~N} 4 \cdots \mathrm{N} 1$ & $2.895(7)$ & $\varphi_{\mathrm{pyr}} \mathrm{N} 3$ & 3.18 \\
$\delta \mathrm{C}_{\mathrm{m}}{ }^{b}$ & 0.074 & $\varphi_{\mathrm{pyr}} \mathrm{N} 4$ & 0.93 \\
$\delta \mathrm{C}_{\alpha}{ }^{e}$ & 0.051 & $\varphi_{\mathrm{ar}} \mathrm{C} 5^{i}$ & 87.05 \\
$\delta \mathrm{C}_{\beta}{ }^{f}$ & 0.102 & $\varphi_{\mathrm{ar}} \mathrm{C} 10$ & 87.87 \\
$\mathrm{C}_{\alpha}-\mathrm{C}_{5}-\mathrm{C}_{\alpha}$ & $126.8(5)$ & $\varphi_{\mathrm{ar}} \mathrm{C} 15$ & 82.98 \\
$\mathrm{C}_{\alpha}-\mathrm{C}_{10}-\mathrm{C}_{\alpha}$ & $124.7(5)$ & $\varphi_{\mathrm{ar}} \mathrm{C} 20$ & 81.15 \\
\hline
\end{tabular}

$\mathrm{C}_{\mathrm{m}}$ (meso carbon) $=5,10,15,20 ; \mathrm{C}_{\beta}(\beta$-pyrrole position $)=2,3,7,8,12,13,17,18 ; \mathrm{C}_{\alpha}(\alpha$-pyrrole position $)=1,4,6,9,11,14,16,19 .{ }^{a}$ The core size is defined as the geometrical centre of the four nitrogen atoms. ${ }^{b}$ The core elongation parameter is defined as the difference between the vector lengths $(|\mathrm{N} 1-\mathrm{N} 2|+|\mathrm{N} 3-\mathrm{N} 4|) / 2-(|\mathrm{N} 2-\mathrm{N} 3|+|\mathrm{N} 1-\mathrm{N} 4|) / 2 .{ }^{c}$ Deviation of the 24 macrocycle atoms from their least squares plane. ${ }^{d}$ Average deviation of the $\mathrm{C}_{\mathrm{m}}$ carbon atoms from the $4 \mathrm{~N}$ plane. ${ }^{e}$ Average deviation of the $\mathrm{C}_{\alpha}$ atoms from the $4 \mathrm{~N}$ plane. ${ }^{f}$ Average deviation of the $\mathrm{C}_{\beta}$ atoms from the $4 \mathrm{~N}$ plane. ${ }^{g} \mathrm{Cis}$ $\mathrm{C}_{\alpha}-\mathrm{N}-\mathrm{N}-\mathrm{C}_{\alpha}$ dihedral angles. ${ }^{11}{ }^{h}$ Pyrrole tilt angle with the $4 \mathrm{~N}$ plane. ${ }^{i}$ Phenyl tilt angle against the $4 \mathrm{~N}$ plane.

Figure 24. Linear display of the skeletal deviations (in units of $0.001 \AA$ ) of the macrocycle atoms from the mean porphyrin plane (of 24 atom).$^{10}$ The $x$ axis is not to scale.

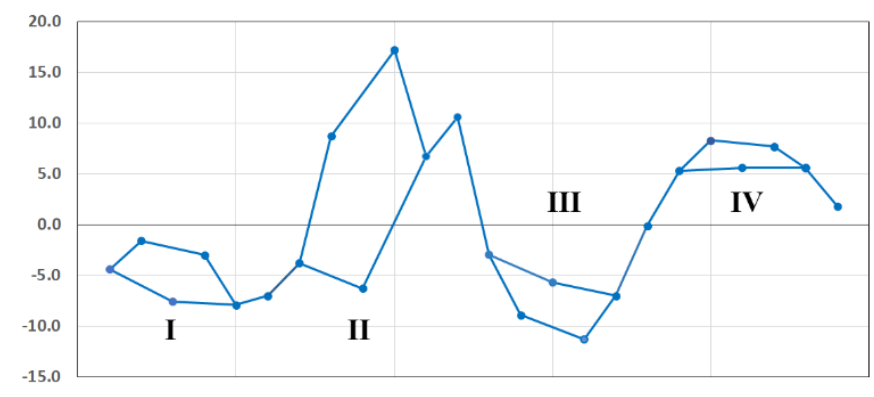

\section{References}

${ }^{1}$ T. A. D. Pinto, R. Hrdina, G. Kirsch, A. M. F. Oliveira-Campos, L. M. Rodrigues and A. P. Esteves, ARKIVOC 2012 , 6, 185-193

${ }^{2}$ H. G. Brittain, Cryst. Growth Des., 2010, 10, 1990-2003

${ }^{3}$ Bruker, SAINT+, 2007, Bruker AXS Inc., Madison, Wisconsin, USA.

${ }^{4}$ Bruker, APEX II, 2009, Bruker AXS Inc., Madison, Wisconsin, USA.

${ }^{5}$ M. C. Burla, R. Caliandro, M. Camalli, B. Carrozzini, G. L. Cascarano, L. De Caro, C. Giacovazzo, G. Polidori and R. Spagna, J. Appl. Crystallogr., 2005, 38, 381-388.

${ }^{6}$ G. M. Sheldrick, Acta Crystallogr. Sect. C Struct. Chem., 2015, 71, 3-8.

${ }^{7}$ L. J. Farrugia, J. Appl. Crystallogr., 2012, 45, 849-854.

${ }^{8}$ K. A. Connors: Binding constants - the measurement of molecular complex stability, John Wiley \& Sons, New York 1987, 91, 1398-1398.

${ }^{9}$ M. Gruden, S. Grubišić, A. G. Coutsolelos and S. R. Niketić, J. Mol. Struct., 2001, 595, 209-224.

${ }^{10}$ a) W. Jentzen, X.-Z. Song and J. A. Shelnutt, J. Phys. Chem. B, 1997, 101, 1684-1699; b) W. Jentzen, J.-G. Ma and J. A. Shelnutt, Biophys. J., 1998, 74, 753-763.

${ }^{11}$ A. Fidalgo-Marijuan, G. Barandika, B. Bazán, M.-K. Urtiaga and M. I. Arriortua, CrystEngComm, $2013,15,4181$. 


\section{Journal Name}

\section{ARTICLE}

\section{Sensing of Diclofenac by a Porphyrin-based Artificial Receptor}

Daniela Intrieri, ${ }^{a}$ Caterina Damiano, ${ }^{a}$ Silvia Rizzato, ${ }^{a}$ Roberto Paolesse, ${ }^{b}$ Mariano Venanzi, ${ }^{\text {b }}$ Donato Monti, ${ }^{\text {b }}$ Marco Savioli, ${ }^{\mathrm{b}}$

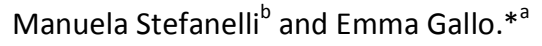

\section{Graphical Abstract}

The synthesis of a porphyrin chemosensor is reported as well as its activity in detecting Diclofenac with an overall $1: 1 K_{\text {binding }} \cong$ $10^{5}$.

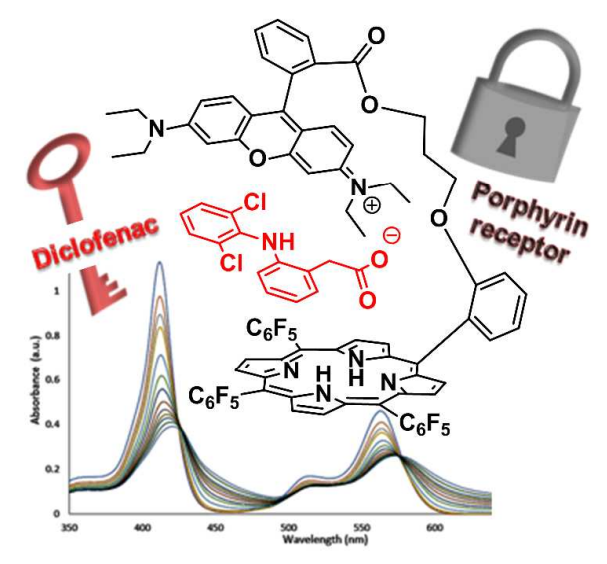




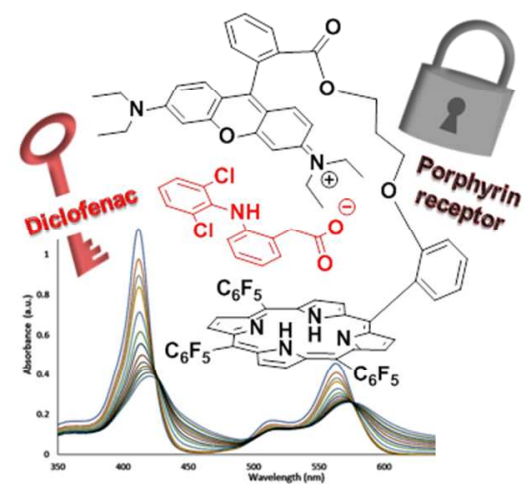

$254 \times 190 \mathrm{~mm}(96 \times 96 \mathrm{DPI})$ 

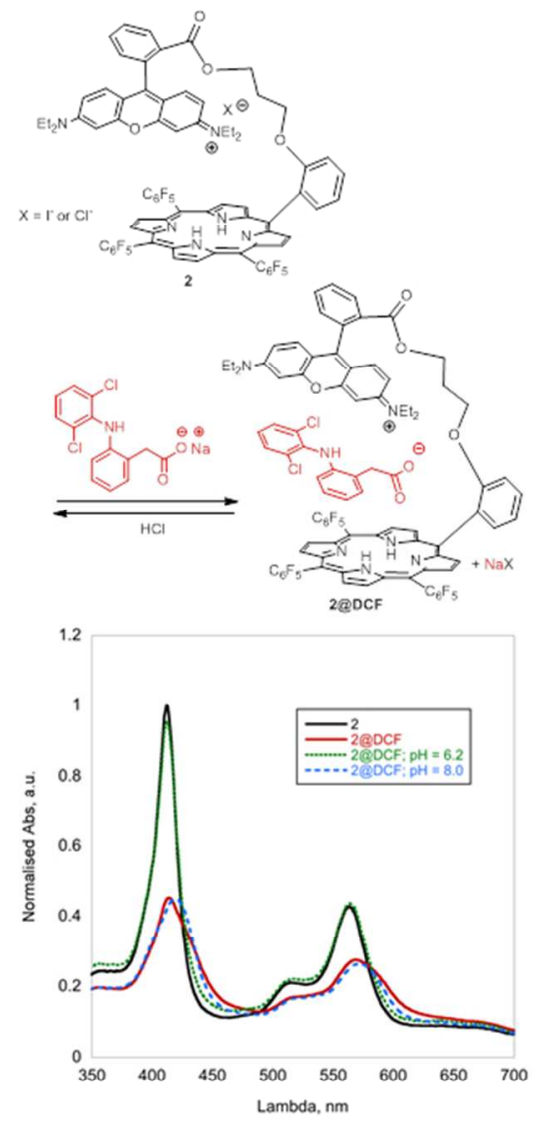

Figure 2. UV-Vis spectra showing the reversible formation of 2@DCF adduct.

$190 \times 254 \mathrm{~mm}(96 \times 96 \mathrm{DPI})$ 


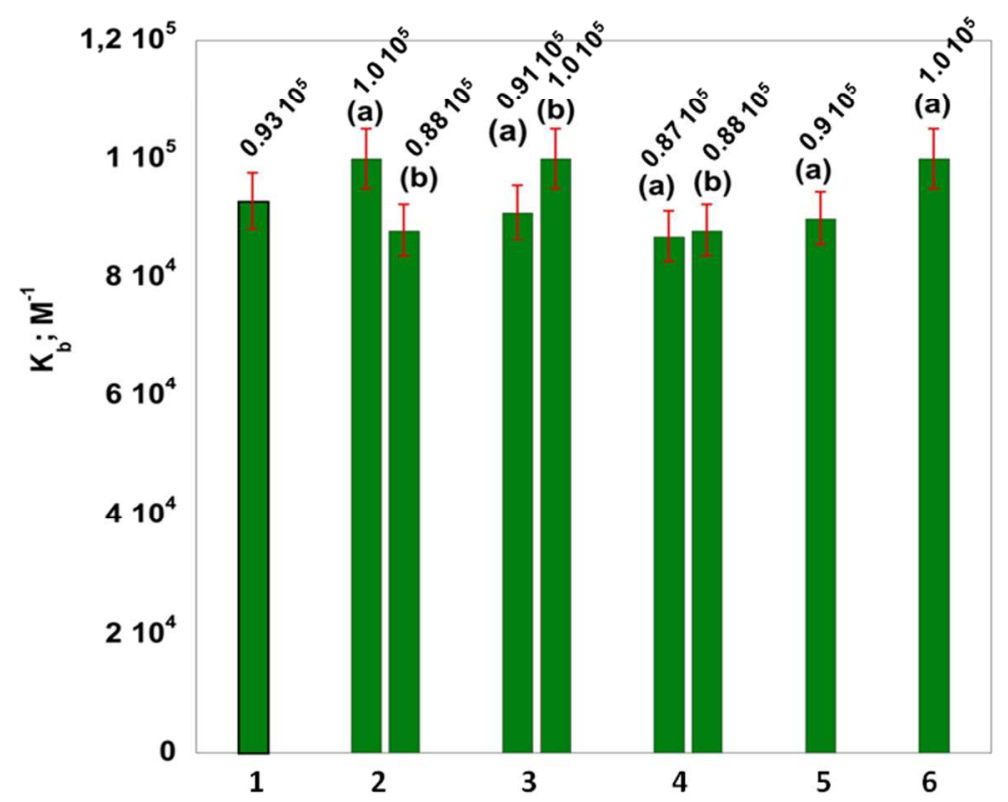

$1=(D C F) N a ; 2$ = sodium phenylacetate; 3 = sodium salicylate;

4 = L-tyrosine sodium salt; 5 = sodium sarcosinate; 6 = L-alanine sodium salt

(a): (DCF) Na/competitor = 1:1; (b): (DCF) Na/competitor $=1: 5$

Figure 6. Binding constant values for the formation of $2 @ D C F$ adduct in the presence of interfering sodium salts. (a): 1:1 molar ratio, (b): 1:5 molar ratio (error bars 5\%).

$254 \times 190 \mathrm{~mm}(96 \times 96 \mathrm{DPI})$ 